\title{
Highly Chemoselective 2,4,5- Triaryl-1,3-dioxolane Formation from Intermolecular 1,3-Dipolar Addition of Carbonyl Ylide with Aryl Aldehydes
}

Chong-Dao Lu, Zhi-Yong Chen, Hui Liu, Wen-Hao Hu*, Ai-Qiao Mi

Key Laboratory for Asymmetric Synthesis and Chirotechnology of Sichuan Province, Chengdu Institute of Organic Chemistry, Chinese Academy of Sciences, Chengdu $610041 \&$ Graduate School of the Chinese Academy of Sciences, Beijing, CHINA huwh@cioc.ac.cn

\section{Supporting Information}

Table of contents:

General procedure for dioxolanation and control reaction (S2)

Table of diagnostic NMR assignments $\left(-\mathrm{COOCH}_{3}\right)$ of compounds 4 and $\mathbf{5}(\mathbf{S 3})$

Analytical data for 4a-m, 5a-5m and methyl $\left(2 R^{*}, 3 R^{*}\right)-2-p h e n y l-3-(2,4-$ dinitrophenyl)-oxirane-2-carboxylate (S4-S13)

X-ray crystal structure of compound $4 \mathrm{~b}(\boldsymbol{S 1 4})$

${ }^{1} \mathrm{H}$ and ${ }^{13} \mathrm{C}$ NMR spectra for all compounds list above (S15-S64) 
General methods. Melting points were determined on a digital melting point apparatus and were uncorrected. HRMS (ESI) Mass spectra were recorded on a BRUCKER FT-MS. NMR spectra were recorded on a Brucker-300MHz spectrometer. Dichloromethane was distilled over calcium hydride before use.

General procedure for the "cross" dioxolanation of phenyldiazoacetate: To a $8 \mathrm{~mL}$ $\mathrm{CH}_{2} \mathrm{Cl}_{2}$ solution of $\mathrm{Rh}_{2}(\mathrm{OAc})_{4}(4.4 \mathrm{mg}, 0.01 \mathrm{mmol})$, $p$-anisaldehyde 2a $(272 \mathrm{mg}, 2.0$ mmol) and p-nitrobenzaldehyde 3a (302 mg, $2.0 \mathrm{mmol}$ ) was added methyl phenyl diazoacetate 1 (176 mg, $1.0 \mathrm{mmol}$ ) in $4 \mathrm{~mL}$ of $\mathrm{CH}_{2} \mathrm{Cl}_{2}$ via a syringe pump over $1 \mathrm{~h}$ under refluxing. After completed addition, the reaction mixture was cooled to room temperature. Solvent was removed, and a portion of the crude product was subjected to ${ }^{1} \mathrm{H}$ NMR analysis for determination of the isomer ratio. The crude product was purified by flash chromatography on silica gel by using petroleum ether:EtOAc $=10: 1$ as eluent to give products $\mathbf{4 a}$ and $\mathbf{5 a}$, total yield 50\%.

Notes: Isomers with larger Rf: 5a, 4b, 4c, 4d, 4e, 4f, 5g, 5h, 5j, 5k, 5 l.

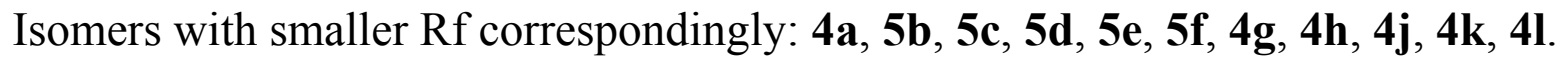

Diastereoisomers $\mathbf{4 i / 5 i}$ and $\mathbf{4 m} / \mathbf{4 m}$ were inseparatable by silica gel chromatography purification, and were characterized as mixtures.

Control reaction (Scheme 4): To a $5 \mathrm{~mL} \mathrm{CH} \mathrm{Cl}_{2}$ solution of $\mathrm{Rh}_{2}(\mathrm{OAc})_{4}(2.2 \mathrm{mg}$, $0.005 \mathrm{mmol}), p$-anisaldehyde 2a (68 mg, $0.5 \mathrm{mmol})$ and epoxide 7 (75 mg, $0.25 \mathrm{mmol})$ was added methyl phenyl diazoacetate $1(88 \mathrm{mg}, 0.5 \mathrm{mmol})$ in $2 \mathrm{~mL}$ of $\mathrm{CH}_{2} \mathrm{Cl}_{2}$ via a syringe pump over $1 \mathrm{~h}$ under refluxing. After completed addition, the reaction mixture was refluxed for additional $1 \mathrm{~h}$ and then cooled to room temperature. Solvent was 
removed, and a portion of the crude product was subjected to ${ }^{1} \mathrm{H}$ NMR analysis, there have no any formation of dioxolanes. (Same procedure was used to the control reaction of $\mathbf{1}$ with $\mathbf{3 a}$ and $\mathbf{6}$, there have no any formation of dioxolanes.)

Table of the diagnostic ${ }^{1} \mathrm{H}$ NMR assignments $\left(-\mathrm{COOCH}_{3}\right)$ of compounds $\mathbf{4}$ and $\mathbf{5}$

\begin{tabular}{|l|l|l|}
\hline & $\mathbf{4}(\delta) \mathrm{ppm}$ & $\mathbf{5}(\delta) \mathrm{ppm}$ \\
\hline $\mathbf{a}$ & $3.89(\mathrm{~s}, 3 \mathrm{H})$ & $3.52(\mathrm{~s}, 3 \mathrm{H})$ \\
\hline $\mathbf{b}$ & $3.95(\mathrm{~s}, 3 \mathrm{H})$ & $3.61(\mathrm{~s}, 3 \mathrm{H})$ \\
\hline $\mathbf{c}$ & $3.95(\mathrm{~s}, 3 \mathrm{H})$ & $3.61(\mathrm{~s}, 3 \mathrm{H})$ \\
\hline $\mathbf{d}$ & $3.94(\mathrm{~s}, 3 \mathrm{H})$ & $3.60(\mathrm{~s}, 3 \mathrm{H})$ \\
\hline $\mathbf{e}$ & $3.92(\mathrm{~s}, 3 \mathrm{H})$ & $3.60(\mathrm{~s}, 3 \mathrm{H})$ \\
\hline $\mathbf{f}$ & $3.95(\mathrm{~s}, 3 \mathrm{H})$ & $3.62(\mathrm{~s}, 3 \mathrm{H})$ \\
\hline $\mathbf{g}$ & $3.86(\mathrm{~s}, 3 \mathrm{H})$ & $3.52(\mathrm{~s}, 3 \mathrm{H})$ \\
\hline $\mathbf{h}$ & $3.85(\mathrm{~s}, 3 \mathrm{H})$ & $3.54(\mathrm{~s}, 3 \mathrm{H})$ \\
\hline $\mathbf{i}$ & $3.78(\mathrm{~s}, 3 \mathrm{H})$ & $3.55(\mathrm{~s}, 3 \mathrm{H})$ \\
\hline $\mathbf{j}$ & $3.84(\mathrm{~s}, 3 \mathrm{H})$ & $3.56(\mathrm{~s}, 3 \mathrm{H})$ \\
\hline $\mathbf{k}$ & $3.83(\mathrm{~s}, 3 \mathrm{H})$ & $3.46(\mathrm{~s}, 3 \mathrm{H})$ \\
\hline $\mathbf{I}$ & $3.85(\mathrm{~s}, 3 \mathrm{H})$ & $3.51(\mathrm{~s}, 3 \mathrm{H})$ \\
\hline $\mathbf{m}$ & $3.86(\mathrm{~s}, 3 \mathrm{H})$ & $3.67(\mathrm{~s}, 3 \mathrm{H})$ \\
\hline
\end{tabular}


Methyl

$\left(2 S^{*}, 4 R^{*}, 5 S^{*}\right)-2-(4-m e t h o x y p h e n y l)-4-p h e n y l-5-(4-n i t r o p h e n y l)-1,3-$

Dioxolane-4-carboxylate, (4a): $\mathrm{mp}$ 105.4-107.0 ${ }^{\circ} \mathrm{C} ;{ }^{1} \mathrm{H}$ NMR $\left(300 \mathrm{MHz}, \mathrm{CDCl}_{3}\right) \delta$ 7.94-7.91 (m, 2H), 7.69-7.66 (m, 2H), 7.37-7.34 (m, 2H), 7.14-7.02 (m, 7H), $6.14(\mathrm{~s}, 1 \mathrm{H})$, $6.00(\mathrm{~s}, 1 \mathrm{H}), 3.89(\mathrm{~s}, 3 \mathrm{H}), 3.88(\mathrm{~s}, 3 \mathrm{H}) ;{ }^{13} \mathrm{C} \mathrm{NMR}\left(75 \mathrm{MHz}, \mathrm{CDCl}_{3}\right) \delta 172.6,161.1,147.5$, $143.8,135.3,128.7,128.6,128.4,128.1,127.4,125.9,122.9,114.2,103.9,88.6,84.3$, 55.5, 53.5; HRMS: calcd for $\mathrm{C}_{24} \mathrm{H}_{21} \mathrm{NO}_{7}$ : 435.1318; found: $435.1324[\mathrm{M}+\mathrm{H}]^{+}$.

Methyl $\quad\left(2 S^{*}, 4 R^{*}, 5 R^{*}\right)-2-(4-m e t h o x y p h e n y l)-4-p h e n y l-5-(4-n i t r o p h e n y l)-1,3-$ Dioxolane-4-carbolate (5a): $\mathrm{mp}$ 148.7-149.5 ${ }^{\circ} \mathrm{C} ;{ }^{1} \mathrm{H}$ NMR $\left(300 \mathrm{MHz}, \mathrm{CDCl}_{3}\right) \delta 8.27$ $8.24(\mathrm{~m}, 2 \mathrm{H}), 7.66-7.63(\mathrm{~m}, 2 \mathrm{H}), 7.58-7.51(\mathrm{~m}, 4 \mathrm{H}), 7.43-7.40(\mathrm{~m}, 3 \mathrm{H}), 6.96-6.93(\mathrm{~m}$, $2 \mathrm{H}), 6.75(\mathrm{~s}, 1 \mathrm{H}), 5.33(\mathrm{~s}, 1 \mathrm{H}), 3.83(\mathrm{~s}, 3 \mathrm{H}), 3.52(\mathrm{~s}, 3 \mathrm{H}) ;{ }^{13} \mathrm{C} \mathrm{NMR}\left(75 \mathrm{MHz}, \mathrm{CDCl}_{3}\right) \delta$ 169.6, 160.7, 148.1, 143.2, 136.1, 130.2, 128.9, 128.6, 128.2, 128.0, 126.4, 123.4, 114.0, 105.5, 89.3, 86.1, 55.4, 52.6; HRMS: calcd for $\mathrm{C}_{24} \mathrm{H}_{21} \mathrm{NO}_{7}$ : 435.1318; found: 435.1311 $[\mathrm{M}+\mathrm{H}]^{+}$.

Methyl $\quad\left(2 S^{*}, 4 R^{*}, 5 S^{*}\right)-2-(4-m e t h o x y p h e n y l)-4-p h e n y l-5-(2,4-d i n i t r o p h e n y l)-1,3-$ Dioxolane-4-carboxylate (4b): $\mathrm{mp} 182.1-183.2{ }^{\circ} \mathrm{C} ;{ }^{1} \mathrm{H}$ NMR $\left(300 \mathrm{MHz}, \mathrm{CDCl}_{3}\right) \delta 8.58$ $(\mathrm{d}, J=2.4 \mathrm{~Hz}, 1 \mathrm{H}), 8.06(\mathrm{dd}, J=8.7,2.4 \mathrm{~Hz}, 1 \mathrm{H}), 7.68-7.64(\mathrm{~m}, 2 \mathrm{H}), 7.58(\mathrm{~d}, J=8.7 \mathrm{~Hz}$, 1H), 7.24-7.21 (m, 2H), 7.10-7.02 (m, 5H), $6.82(\mathrm{~s}, 1 \mathrm{H}), 6.26(\mathrm{~s}, 1 \mathrm{H}), 3.95(\mathrm{~s}, 3 \mathrm{H}), 3.89$ $(\mathrm{s}, 3 \mathrm{H}) ;{ }^{13} \mathrm{C} \mathrm{NMR}\left(75 \mathrm{MHz}, \mathrm{CDCl}_{3}\right) \delta 171.0,161.1,148.2,147.1,140.0,134.6,132.0$, $128.8,128.4,128.2,127.1,126.5,126.1,119.5,114.3,104.1,89.2,79.8,55.5,53.7$; HRMS: calcd for $\mathrm{C}_{24} \mathrm{H}_{20} \mathrm{~N}_{2} \mathrm{O}_{9}$ : 480.1169; found: $480.1174[\mathrm{M}+\mathrm{H}]^{+}$. Single crystal was grown in hexane/ethyl actate and corresponding X-ray structure was obtained. 
Methyl $\left(2 S^{*}, 4 R^{*}, 5 R^{*}\right)$-2-(4-methoxyphenyl)-4-phenyl-5-(2,4-dinitrophenyl)-1,3Dioxolane-4-carboxylate (5b): ${ }^{1} \mathrm{H}$ NMR $\left(300 \mathrm{MHz}, \mathrm{CDCl}_{3}\right) \delta 8.72(\mathrm{~d}, J=2.4 \mathrm{~Hz}, 1 \mathrm{H})$, $8.44(\mathrm{dd}, J=8.7,2.4 \mathrm{~Hz}, 1 \mathrm{H}), 7.90(\mathrm{~d}, J=8.7 \mathrm{~Hz}, 1 \mathrm{H}), 7.58-7.54(\mathrm{~m}, 2 \mathrm{H}), 7.52-7.49(\mathrm{~m}$, 2H), 7.41-7.35 (m, 3H), 6.97-6.93 (m, 2H), $6.54(\mathrm{~s}, 1 \mathrm{H}), 6.20(\mathrm{~s}, 1 \mathrm{H}), 3.84(\mathrm{~s}, 3 \mathrm{H}), 3.61$ (s, 3H); ${ }^{13} \mathrm{C}$ NMR $\left(75 \mathrm{MHz}, \mathrm{CDCl}_{3}\right) \delta 169.2,160.9,148.7,147.7,138.3,135.7,130.7$, $129.4,129.1,128.9,128.3,126.6,126.0,120.4,114.1,106.0,90.1,83.0,55.5,53.0$; HRMS: calcd for $\mathrm{C}_{24} \mathrm{H}_{20} \mathrm{~N}_{2} \mathrm{O}_{9}$ : 480.1169; found: $480.1165[\mathrm{M}+\mathrm{H}]^{+}$.

Methyl $\quad\left(2 S^{*}, 4 R^{*}, 5 S^{*}\right)-2-(2-m e t h o x y p h e n y l)-4-p h e n y l-5-(2,4-d i n i t r o p h e n y l)-1,3-$ Dioxolane-4-carboxylate (4c): $\mathrm{mp} 144.8-146.2{ }^{\circ} \mathrm{C} ;{ }^{1} \mathrm{H} \mathrm{NMR}\left(300 \mathrm{MHz}, \mathrm{CDCl}_{3}\right) \delta 8.54$ (d, $J=2.4 \mathrm{~Hz}, 1 \mathrm{H}), 8.06(\mathrm{dd}, J=8.7,2.4 \mathrm{~Hz}, 1 \mathrm{H}), 7.92-7.89(\mathrm{~m}, 1 \mathrm{H}), 7.64(\mathrm{~d}, J=8.7 \mathrm{~Hz}$, 1H), 7.52-7.46 (m, 1H), 7.29-7.26 (m, 2H), 7.18-7.01 (m, 5H), $6.90(\mathrm{~s}, 1 \mathrm{H}), 6.56(\mathrm{~s}, 1 \mathrm{H})$, $3.95(\mathrm{~s}, 3 \mathrm{H}), 3.92(\mathrm{~s}, 3 \mathrm{H}) ;{ }^{13} \mathrm{C} \mathrm{NMR}\left(75 \mathrm{MHz}, \mathrm{CDCl}_{3}\right) \delta 171.0,158.2,148.1,147.0$, $140.3,134.5,132.2,131.6,128.7,128.2,127.1,126.4,126.0,122.8,120.8,119.4,111.3$, 100.0, 89.2, 79.5, 55.9, 53.7; HRMS: calcd for $\mathrm{C}_{24} \mathrm{H}_{20} \mathrm{~N}_{2} \mathrm{O}_{9}$ : 480.1169; found: 480.1164 $\left[\mathrm{M}+\mathrm{NH}_{4}\right]^{+}$.

(Methyl $\left(2 S^{*}, 4 R^{*}, 5 R^{*}\right)-2-(2-m e t h o x y p h e n y l)-4-p h e n y l-5-(2,4-d i n i t r o p h e n y l)-1,3-$ Dioxolane-4-carboxylate (5c): $\mathrm{mp} 126.6-128.7{ }^{\circ} \mathrm{C} ;{ }^{1} \mathrm{H} \mathrm{NMR}\left(300 \mathrm{MHz}, \mathrm{CDCl}_{3}\right) \delta 8.74$ (d, $J=2.4 \mathrm{~Hz}, 1 \mathrm{H}), 8.45(\mathrm{dd}, J=8.7,2.4 \mathrm{~Hz}, 1 \mathrm{H}), 7.99$ (d, $J=8.7 \mathrm{~Hz}, 1 \mathrm{H}), 7.60-7.53$ (m, 3H), 7.43-7.35 (m, 4H), 7.01-6.96 (m, 2H), $6.83(\mathrm{~s}, 1 \mathrm{H}), 6.32(\mathrm{~s}, 1 \mathrm{H}), 3.93(\mathrm{~s}, 3 \mathrm{H}), 3.61$ (s, 3H); ${ }^{13} \mathrm{C}$ NMR $\left(75 \mathrm{MHz}, \mathrm{CDCl}_{3}\right) \delta 169.3,157.9,148.7,147.6,138.8,135.9,131.3$, $130.8,129.3,128.8,127.8,126.6,126.1,124.6,120.6,120.4,111.1,102.8,90.1,82.9$, 55.8, 52.9; HRMS: calcd for $\mathrm{C}_{24} \mathrm{H}_{20} \mathrm{~N}_{2} \mathrm{O}_{9}$ : 480.1169; found: 480.1173 [M+ $\left.\mathrm{NH}_{4}\right]^{+}$. 
Methyl $\left(2 S^{*}, 4 R^{*}, 5 S^{*}\right)-2$-piperonyl-4-phenyl-5-(2,4-dinitrophenyl)-1,3-Dioxolane4-carboxylate (4d): $\mathrm{mp} 145.7-147.2{ }^{\circ} \mathrm{C} ;{ }^{1} \mathrm{H}$ NMR $\left(300 \mathrm{MHz}, \mathrm{CDCl}_{3}\right) \delta 8.58(\mathrm{~d}, J=2.4$ $\mathrm{Hz}, 1 \mathrm{H}), 8.06$ (dd, $J=8.7,2.4 \mathrm{~Hz}, 1 \mathrm{H}), 7.55(\mathrm{~d}, J=8.7 \mathrm{~Hz}, \mathrm{H}), 7.22-7.17$ (m, 4H), 7.10$7.04(\mathrm{~m}, 3 \mathrm{H}), 6.92(\mathrm{~d}, J=7.8 \mathrm{~Hz}, 1 \mathrm{H}), 6.79(\mathrm{~s}, 1 \mathrm{H}), 6.20(\mathrm{~s}, 1 \mathrm{H}), 6.06(\mathrm{~s}, 2 \mathrm{H}), 3.94(\mathrm{~s}$, $3 \mathrm{H}) ;{ }^{13} \mathrm{C}$ NMR $\left(75 \mathrm{MHz}, \mathrm{CDCl}_{3}\right) \delta 170.9,149.2,148.1,148.0,147.0,139.7,134.4,131.9$, $128.7,128.6,128.1,126.5126 .0,121.3,119.5,108.5,107.0,103.8,101.6,89.0,79.8$, 53.7; HRMS: calcd for $\mathrm{C}_{24} \mathrm{H}_{18} \mathrm{~N}_{2} \mathrm{O}_{10}$ : 494.0962; found: $494.0959[\mathrm{M}+\mathrm{H}]^{+}$.

\section{Methyl $\left(2 S^{*}, 4 R^{*}, 5 R^{*}\right)-2$-piperonyl-4-phenyl-5-(2,4-dinitrophenyl)-1,3-Dioxolane-}

4-carboxylate (5d): $\mathrm{mp} 172.1-172.9{ }^{\circ} \mathrm{C} ;{ }^{1} \mathrm{H}$ NMR $\left(300 \mathrm{MHz}, \mathrm{CDCl}_{3}\right) \delta 8.71(\mathrm{~d}, J=2.4$ $\mathrm{Hz}, 1 \mathrm{H}), 8.44(\mathrm{dd}, J=8.7,2.4 \mathrm{~Hz}, 1 \mathrm{H}), 7.88(\mathrm{~d}, J=8.7 \mathrm{~Hz}, 1 \mathrm{H}), 7.57-7.54(\mathrm{~m}, 2 \mathrm{H}), 7.41-$ $7.36(\mathrm{~m}, 3 \mathrm{H}), 7.08-7.04(\mathrm{~m}, 2 \mathrm{H}), 6.84(\mathrm{~d}, J=7.8 \mathrm{~Hz}, 1 \mathrm{H}), 6.48(\mathrm{~s}, 1 \mathrm{H}), 6.18(\mathrm{~s}, 1 \mathrm{H}), 6.00$ (s, 2H), $3.60(\mathrm{~s}, 3 \mathrm{H}) ;{ }^{13} \mathrm{C}$ NMR $\left(75 \mathrm{MHz}, \mathrm{CDCl}_{3}\right) \delta 169.1,149.0,148.6,148.1,147.7$, $138.1,135.5,130.8,130.7,129.5,128.9,126.6,125.9,121.1,120.4,108.3,106.9,105.8$, 101.5, 90.1, 83.0, 53.0; HRMS: calcd for $\mathrm{C}_{24} \mathrm{H}_{18} \mathrm{~N}_{2} \mathrm{O}_{10}$ : 494.0962; found: 494.0962 $[\mathrm{M}+\mathrm{H}]^{+}$.

Methyl $\quad\left(2 S^{*}, 4 R^{*}, 5 S^{*}\right)-2-(3-m e t h y l p h e n y l)-4-p h e n y l-5-(2,4-d i n i t r o p h e n y l)-1,3-$ Dioxolane-4-carboxylate (4e): $\mathrm{mp} 137.5-138.7{ }^{\circ} \mathrm{C} ;{ }^{1} \mathrm{H} \mathrm{NMR}\left(300 \mathrm{MHz}, \mathrm{CDCl}_{3}\right) \delta 8.53$ (d, $J=2.4 \mathrm{~Hz}, 1 \mathrm{H}), 8.02(\mathrm{dd}, J=8.7,2.4 \mathrm{~Hz}, 1 \mathrm{H}), 7.57-7.50(\mathrm{~m}, 3 \mathrm{H}), 7.42-7.39(\mathrm{~m}, 1 \mathrm{H})$, 7.31-7.29 (m, 1H), 7.23-7.19 (m, 2H), 7.07-7.02 (m, 3H), 6.82 (s, 1H), 6.24 (s, 1H), 3.92 (s, 3H), $2.44(\mathrm{~s}, 3 \mathrm{H}) ;{ }^{13} \mathrm{C}$ NMR $\left(75 \mathrm{MHz}, \mathrm{CDCl}_{3}\right) \delta 170.8,148.0,146.9,139.8,138.6$, $134.8,134.4,131.9,130.8,128.7,128.6,128.0,127.5,126.4,126.0,123.6,119.4,103.9$, 89.1, 79.7, 53.5, 21.5; HRMS: calcd for $\mathrm{C}_{24} \mathrm{H}_{20} \mathrm{~N}_{2} \mathrm{O}_{8}$ : 464.1220; found: $464.1228[\mathrm{M}+\mathrm{H}]^{+}$. 
Methyl $\quad\left(2 S^{*}, 4 R^{*}, 5 R^{*}\right)-2-(3-m e t h y l p h e n y l)-4-p h e n y l-5-(2,4-d i n i t r o p h e n y l)-1,3-$ Dioxolane-4-carboxylate (5e): ${ }^{1} \mathrm{H}$ NMR $\left(300 \mathrm{MHz}, \mathrm{CDCl}_{3}\right) \delta 8.70(\mathrm{~d}, J=2.4 \mathrm{~Hz}, 1 \mathrm{H})$, $8.42(\mathrm{dd}, J=8.7,2.4 \mathrm{~Hz}, 1 \mathrm{H}), 7.90(\mathrm{~d}, J=8.7 \mathrm{~Hz}, 1 \mathrm{H}), 7.56-7.53(\mathrm{~m}, 2 \mathrm{H}), 7.38-7.30(\mathrm{~m}$, $6 \mathrm{H}), 7.23-7.20(\mathrm{~m}, 1 \mathrm{H}), 6.53(\mathrm{~s}, 1 \mathrm{H}), 6.19(\mathrm{~s}, 1 \mathrm{H}), 3.60(\mathrm{~s}, 3 \mathrm{H}), 2.37(\mathrm{~s}, 3 \mathrm{H}) ;{ }^{13} \mathrm{C}$ NMR $\left(75 \mathrm{MHz}, \mathrm{CDCl}_{3}\right) \delta 169.0,147.5,138.4,138.1,136.8,135.4,130.6,130.5,129.3,128.7$, $128.5,128.0,127.2,126.4,125.9,123.6,120.3,105.9,90.0,82.9,52.8,21.4$; HRMS: calcd for $\mathrm{C}_{24} \mathrm{H}_{20} \mathrm{~N}_{2} \mathrm{O}_{8}$ : 464.1220; found: $464.1225[\mathrm{M}+\mathrm{H}]^{+}$.

Methyl $\left(2 S^{*}, 4 R^{*}, 5 S^{*}\right)-2,4-d i p h e n y l-5-(2,4-d i n i t r o p h e n y l)-1,3-D i o x o l a n e-4-$ carboxylate (4f): $\mathrm{mp} 171.5-172.5{ }^{\circ} \mathrm{C} ;{ }^{1} \mathrm{H} \mathrm{NMR}\left(300 \mathrm{MHz}, \mathrm{CDCl}_{3}\right) \delta 8.57$ (d, $J=2.4 \mathrm{~Hz}$, $1 \mathrm{H}), 8.05(\mathrm{dd}, J=8.7,2.4 \mathrm{~Hz}, 1 \mathrm{H}), 7.75-7.72(\mathrm{~m}, 2 \mathrm{H}), 7.56(\mathrm{~d}, J=8.7 \mathrm{~Hz}, 1 \mathrm{H}), 7.55-$ $7.51(\mathrm{~m}, 3 \mathrm{H}), 7.24-7.20(\mathrm{~m}, 2 \mathrm{H}), 7.10-7.04(\mathrm{~m}, 3 \mathrm{H}), 6.85(\mathrm{~s}, 1 \mathrm{H}), 6.30(\mathrm{~s}, 1 \mathrm{H}), 3.95(\mathrm{~s}$, $3 \mathrm{H}) ;{ }^{13} \mathrm{C}$ NMR $\left(75 \mathrm{MHz}, \mathrm{CDCl}_{3}\right) \delta 170.9,148.1,147.1,139.9,135.0,134.4,132.0,130.2$, $128.9,128.8,128.2,126.8,126.5,126.1,119.5,104.0,89.3,79.8$ 53.7; HRMS: calcd for $\mathrm{C}_{23} \mathrm{H}_{18} \mathrm{~N}_{2} \mathrm{O}_{8}: 450.1063$; found: $450.1064[\mathrm{M}+\mathrm{H}]^{+}$.

\section{Methyl $\quad\left(2 S^{*}, 4 R^{*}, 5 R^{*}\right)-2,4-d i p h e n y l-5-(2,4-d i n i t r o p h e n y l)-1,3-D i o x o l a n e-4-$} carboxylate (5f): $\mathrm{mp} 134.0-135.7{ }^{\circ} \mathrm{C} ;{ }^{1} \mathrm{H} \mathrm{NMR}\left(300 \mathrm{MHz}, \mathrm{CDCl}_{3}\right) \delta 8.73(\mathrm{~d}, J=2.4 \mathrm{~Hz}$, 1H), 8.44 (dd, $J=8.7,2.4 \mathrm{~Hz}, 1 \mathrm{H}), 7.92$ (d, $J=8.7 \mathrm{~Hz}, 1 \mathrm{H}), 7.59-7.54(\mathrm{~m}, 4 \mathrm{H}), 7.45-$ $7.26(\mathrm{~m}, 6 \mathrm{H}), 6.59(\mathrm{~s}, 1 \mathrm{H}), 6.21(\mathrm{~s}, 1 \mathrm{H}), 3.62(\mathrm{~s}, 3 \mathrm{H}) ;{ }^{13} \mathrm{C} \mathrm{NMR}\left(75 \mathrm{MHz}, \mathrm{CDCl}_{3}\right) \delta$ $169.1,148.6,147.7,138.1,137.0,135.4,130.8,130.0,129.5,128.9,128.7,126.7,126.6$, 126.0, 120.4, 105.9, 90.2, 83.0, 53.0; HRMS: calcd for $\mathrm{C}_{23} \mathrm{H}_{18} \mathrm{~N}_{2} \mathrm{O}_{8}$ : 450.1063; found: $450.1066\left[\mathrm{M}+\mathrm{NH}_{4}\right]^{+}$. 
Methyl $\quad\left(2 S^{*}, 4 R^{*}, 5 S^{*}\right)-2-(2,4-d i m e t h o x y p h e n y l)-4-p h e n y l-5-(4-n i t r o p h e n y l)-1,3-$

Dioxolane-4-carboxylate (4g): $\mathrm{mp} 169.8-170.5{ }^{\circ} \mathrm{C} ;{ }^{1} \mathrm{H}$ NMR $\left(300 \mathrm{MHz}, \mathrm{CDCl}_{3}\right) \delta$ 7.92$7.89(\mathrm{~m}, 2 \mathrm{H}), 7.83(\mathrm{~d}, J=8.4 \mathrm{~Hz}, 1 \mathrm{H}), 7.36-7.33(\mathrm{~m}, 2 \mathrm{H}), 7.18-7.15(\mathrm{~m}, 2 \mathrm{H}), 7.08-7.03$ (m, 3H), $6.65(\mathrm{dd}, J=8.4,2.4 \mathrm{~Hz}, 1 \mathrm{H}), 6.53(\mathrm{~d}, J=2.4 \mathrm{~Hz}, 1 \mathrm{H}), 6.43(\mathrm{~s}, 1 \mathrm{H}), 6.00$ (s, 1H), 3.88 (s, 3H), 3.87 (s, 3H), 3.86 (s, 3H); ${ }^{13} \mathrm{C} \mathrm{NMR}\left(75 \mathrm{MHz}, \mathrm{CDCl}_{3}\right) \delta$ 172.7, 162.4, $159.4,147.3,144.2,135.4,128.7,128.4,128.3,128.0,125.9,122.8,115.8,104.7,99.5$, 98.7, 88.4, 84.1, 55.8, 55.5, 53.4; HRMS: calcd for $\mathrm{C}_{25} \mathrm{H}_{23} \mathrm{NO}_{8}$ : 465.1424; found: $465.1418[\mathrm{M}+\mathrm{H}]^{+}$.

\section{Methyl $\left(2 S^{*}, 4 R^{*}, 5 R^{*}\right)-2-(2,4-d i m e t h o x y p h e n y l)-4-p h e n y l-5-(4-n i t r o p h e n y l)-1,3-$}

Dioxolane-4-carboxylate (5g): $\mathrm{mp} 121.7-122.4{ }^{\circ} \mathrm{C} ;{ }^{1} \mathrm{H}$ NMR $\left(300 \mathrm{MHz}, \mathrm{CDCl}_{3}\right) \delta 8.26-$ $8.22(\mathrm{~m}, 2 \mathrm{H}), 7.68-7.64(\mathrm{~m}, 2 \mathrm{H}), 7.59-7.56(\mathrm{~m}, 2 \mathrm{H}), 7.48-7.38(\mathrm{~m}, 4 \mathrm{H}), 7.00(\mathrm{~s}, 1 \mathrm{H})$, 6.51-6.48 (m, 2H), $5.35(\mathrm{~s}, 1 \mathrm{H}), 3.87(\mathrm{~s}, 3 \mathrm{H}), 3.82(\mathrm{~s}, 3 \mathrm{H}), 3.52(\mathrm{~s}, 3 \mathrm{H}) ;{ }^{13} \mathrm{C}$ NMR $(75$ $\left.\mathrm{MHz}, \mathrm{CDCl}_{3}\right) \delta 169.8,162.2,159.2,148.1,143.6,136.4,128.8,128.6,128.5,128.4$, 126.5, 123.4, 118.3, 104.3, 102.2, 98.8, 89.1, 85.9, 55.7, 55.5, 52.5; HRMS: calcd for $\mathrm{C}_{25} \mathrm{H}_{23} \mathrm{NO}_{8}$ : 465.1424; found: $465.1424[\mathrm{M}+\mathrm{H}]^{+}$.

Methyl $\left(2 S^{*}, 4 R^{*}, 5 S^{*}\right)-2-(2,4,6-t r i m e t h o x y p h e n y l)-4-p h e n y l-5-(4-n i t r o p h e n y l)-1,3-$ Dioxolane-4-carboxylate (4h): $\mathrm{mp} 195.6-196.5{ }^{\circ} \mathrm{C} ;{ }^{1} \mathrm{H} \mathrm{NMR}\left(300 \mathrm{MHz}, \mathrm{CDCl}_{3}\right) \delta$ 7.95$7.90(\mathrm{~m}, 2 \mathrm{H}), 7.49-7.45(\mathrm{~m}, 2 \mathrm{H}), 7.31-7.28(\mathrm{~m}, 2 \mathrm{H}), 7.05-7.01(\mathrm{~m}, 3 \mathrm{H}), 6.62(\mathrm{~s}, 1 \mathrm{H})$, $6.20(\mathrm{~s}, 2 \mathrm{H}), 5.93(\mathrm{~s}, 1 \mathrm{H}), 3.89$ (s, 6H), 3.87 (s, 3H), 3.85 (s, 3H); ${ }^{13} \mathrm{C}$ NMR $(75 \mathrm{MHz}$, $\left.\mathrm{CDCl}_{3}\right) \delta 173.6,163.1,161.3,147.3,145.0,135.9,128.8,128.0,127.6,126.2,122.7$ 102.3, 98.7, 90.9, 87.9, 84.0, 55.6, 55.5, 53.3; HRMS: calcd for $\mathrm{C}_{26} \mathrm{H}_{25} \mathrm{NO}_{9}$ : 495.1529; found: $495.1522[\mathrm{M}+\mathrm{H}]^{+}$. 
Methyl $\left(2 S^{*}, 4 R^{*}, 5 R^{*}\right)-2-(2,4,6-t r i m e t h o x y p h e n y l)-4-p h e n y l-5-(4-n i t r o p h e n y l)-1,3-$

Dioxolane-4-carboxylate (5h): $\mathrm{mp}$ 190.0-190.9 ${ }^{\circ} \mathrm{C} ;{ }^{1} \mathrm{H}$ NMR $\left(300 \mathrm{MHz}, \mathrm{CDCl}_{3}\right) \delta 8.24-$ $8.19(\mathrm{~m}, 2 \mathrm{H}), 7.69-7.66(\mathrm{~m}, 2 \mathrm{H}), 7.57-7.54(\mathrm{~m}, 2 \mathrm{H}), 7.42-7.36(\mathrm{~m}, 3 \mathrm{H}), 7.31(\mathrm{~s}, 1 \mathrm{H})$, $6.15(\mathrm{~s}, 2 \mathrm{H}), 5.48(\mathrm{~s}, 1 \mathrm{H}), 3.82(\mathrm{~s}, 3 \mathrm{H}), 3.76(\mathrm{~s}, 6 \mathrm{H}), 3.54(\mathrm{~s}, 3 \mathrm{H}) ;{ }^{13} \mathrm{C}$ NMR $(75 \mathrm{MHz}$, $\left.\mathrm{CDCl}_{3}\right) \delta 170.0,162.8,160.7,147.9,144.5,136.4,128.6,128.3,128.2,126.6,123.2$ 105.7, 100.5, 90.9, 89.5, 86.9, 55.8, 55.4, 52.4; HRMS: calcd for $\mathrm{C}_{26} \mathrm{H}_{25} \mathrm{NO}_{9}$ : 495.1529; found: $495.1525[\mathrm{M}+\mathrm{H}]^{+}$.

Methyl $\left(2 S^{*}, 4 R^{*}, 5 S^{*}\right)-2-(2,4,6-t r i m e t h o x y p h e n y l)-4-p h e n y l-5-(2-n i t r o p h e n y l)-1,3-$ Dioxolane-4-carboxylate and Methyl $\left(2 S^{*}, 4 R^{*}, 5 R^{*}\right)-2-(2,4,6$-trimethoxyphenyl)-4phenyl-5-(2-nitrophenyl)-1,3-Dioxolane-4-carboxylate (4i+5i) (mixture of diastereomers) ${ }^{1} \mathrm{H}$ NMR $\left(300 \mathrm{MHz}, \mathrm{CDCl}_{3}\right) \delta(7.74-7.70(\mathrm{~m}, 1 \mathrm{H})$; minor diastereomer, 5i), (7.70-7.69 (m, 1H)), 7.76-7.72 (m, 1H; major diastereomer, 4i), (7.66-7.62 (m, 2H)), 7.66-7.65 (m, 1H), (7.59-7.56 (m, 1H)), (7.48-7.42 (m, 1H)), 7.38-7.30 (m, 4H), (7.22 (s, 1H)), (7.20-7.15 (m, 3H)), 7.02-6.99 (m, 3H), $6.81(\mathrm{~s}, 1 \mathrm{H}), 6.55$ (s, 1H), (6.28 (s, 1H)), $6.19(\mathrm{~s}, 2 \mathrm{H}),(6.13(\mathrm{~s}, 2 \mathrm{H})),(3.89(\mathrm{~s}, 6 \mathrm{H})), 3.88(\mathrm{~s}, 6 \mathrm{H}), 3.83(\mathrm{~s}, 3 \mathrm{H}),(3.81(\mathrm{~s}, 3 \mathrm{H})), 3.78$ (s, 3H), (3.55 (s, 3H)); ${ }^{13} \mathrm{C}$ NMR (75 MHz, $\left.\mathrm{CDCl}_{3}\right) \delta$ 172.2(170.0), 163.0(162.6), 161.3(160.7), 148.6(149.2), 136.4(136.2), 133.4(131.5), 132.3(132.2), 130.8(129.6), 128.3(129.0), 128.1(128.5), 127.1(127.5), 126.7(126.2), 123.4(124.7), 100.4(105.8), 98.6(102.4), $90.9(90.8), \quad 87.7(89.6), \quad 80.0(82.7), \quad 55.7(55.4), \quad 55.5(53.1), \quad 55.4(52.3)$; HRMS: calcd for $\mathrm{C}_{26} \mathrm{H}_{25} \mathrm{NO}_{9}$ : 495.1529; found: $495.1529[\mathrm{M}+\mathrm{H}]^{+}$.

Methyl $\left(2 S^{*}, 4 R^{*}, 5 S^{*}\right)-2-(2,4,6-t r i m e t h o x y p h e n y l)-4-p h e n y l-5-(2-f o r m y l p h e n y l)-$ 1,3-Dioxolane-4-carboxylate (4j): ${ }^{1} \mathrm{H}$ NMR $\left(300 \mathrm{MHz}, \mathrm{CDCl}_{3}\right) \delta 10.46(\mathrm{~s}, 1 \mathrm{H}), 7.68$ - 
$7.61(\mathrm{~m}, 2 \mathrm{H}), 7.34-7.20(\mathrm{~m}, 4 \mathrm{H}), 7.03-6.99(\mathrm{~m}, 3 \mathrm{H}), 6.69(\mathrm{~s}, 1 \mathrm{H}), 6.46(\mathrm{~s}, 1 \mathrm{H}), 6.19(\mathrm{~s}$, 2H), 3.89 (s, 6H), 3.85 (s, 3H), 3.84 (s, 3H); ${ }^{13} \mathrm{C} \mathrm{NMR}\left(75 \mathrm{MHz}, \mathrm{CDCl}_{3}\right) \delta$ 192.9, 173.5, $163.1,161.3,138.7,136.1,135.0,133.0,130.4,128.6,128.3,127.8,127.4,126.6,102.2$, 98.6, 90.9, 87.3, 83.3, 55.7, 55.5, 53.2; HRMS: calcd for $\mathrm{C}_{27} \mathrm{H}_{26} \mathrm{O}_{8}$ : 478.1628; found: $478.1627[\mathrm{M}+\mathrm{H}]^{+}$.

\section{Methyl $\quad\left(2 S^{*}, 4 R^{*}, 5 R^{*}\right)-2-(2,4,6$-trimethoxyphenyl)-4-phenyl-5-(2-formylphenyl)-} 1,3-Dioxolane-4-carboxylate (5j): $\mathrm{mp} 126.5-127.8{ }^{\circ} \mathrm{C} ;{ }^{1} \mathrm{H}$ NMR $\left(300 \mathrm{MHz}, \mathrm{CDCl}_{3}\right) \delta$ $9.87(\mathrm{~s}, 1 \mathrm{H})$, 7.95-7.92 (m, 1H), 7.73-7.58 (m, 4H), 7.51-7.46 (m, 1H), 7.35-7.31 (m, 3H), $6.26(\mathrm{~s}, 1 \mathrm{H}), 6.16(\mathrm{~s}, 2 \mathrm{H}), 3.82(\mathrm{~s}, 3 \mathrm{H}), 3.79(\mathrm{~s}, 6 \mathrm{H}), 3.56(\mathrm{~s}, 3 \mathrm{H}) ;{ }^{13} \mathrm{C}$ NMR $(75 \mathrm{MHz}$ $\left.\mathrm{CDCl}_{3}\right) \delta 190.6,170.1,162.6,160.7,138.4,135.6,134.6,133.3,129.5,128.62,128.64$ (overlap, 2C), 128.2, 126.6, 106.1, 100.0, 90.8, 89.7, 83.3, 55.7, 55.4, 52.3; HRMS: calcd for $\mathrm{C}_{27} \mathrm{H}_{26} \mathrm{O}_{8}$ : 478.1628; found: $478.1625[\mathrm{M}+\mathrm{H}]^{+}$.

Methyl $\left(2 S^{*}, 4 R^{*}, 5 S^{*}\right)-2-(2,4,6-$ trimethoxyphenyl)-4-phenyl-5-(4trifluoromethylphenyl)-1,3-Dioxolane-4-carboxylate (4k): $\mathrm{mp} \quad 132.0-133.5 \quad{ }^{\circ} \mathrm{C} ;{ }^{1} \mathrm{H}$ NMR $\left(300 \mathrm{MHz}, \mathrm{CDCl}_{3}\right) \delta 7.40-7.25(\mathrm{~m}, 6 \mathrm{H}), 7.03-7.01(\mathrm{~m}, 3 \mathrm{H}), 6.62(\mathrm{~s}, 1 \mathrm{H}), 6.18(\mathrm{~s}$, $2 \mathrm{H}), 5.88(\mathrm{~s}, 1 \mathrm{H}), 3.87(\mathrm{~s}, 6 \mathrm{H}), 3.84(\mathrm{~s}, 3 \mathrm{H}), 3.83(\mathrm{~s}, 3 \mathrm{H}) ;{ }^{19} \mathrm{~F}\left(282 \mathrm{MHz}, \mathrm{CDCl}_{3}\right) \delta-$ 62.52: ${ }^{13} \mathrm{C}$ NMR $\left(75 \mathrm{MHz}, \mathrm{CDCl}_{3}\right) \delta 173.7,163.0,161.3,141.3,136.1,129.6\left(\mathrm{q},{ }^{2} J(\mathrm{C}, \mathrm{F})\right.$ $=32 \mathrm{~Hz}), 128.4,127.6,127.3,126.3,124.3\left(\mathrm{q},{ }^{3} J(\mathrm{C}, \mathrm{F})=4 \mathrm{~Hz}\right), 124.1\left(\mathrm{q},{ }^{1} J(\mathrm{C}, \mathrm{F})=270\right.$ $\mathrm{Hz})$, 102.6, 98.5, 90.9, 87.6, 84.5, 55.5, 55.4, 53.1; HRMS: calcd for $\mathrm{C}_{27} \mathrm{H}_{25} \mathrm{~F}_{3} \mathrm{O}_{7}$ : 518.1552; found: $518.1553[\mathrm{M}+\mathrm{H}]^{+}$.

Methyl $\left(2 S^{*}, 4 R^{*}, 5 R^{*}\right)-2-(2,4,6-$ trimethoxyphenyl)-4-phenyl-5-(4trifluoromethylphenyl)-1,3-Dioxolane-4-carboxylate (5k): mp $173.5-174.3 \quad{ }^{\circ} \mathrm{C} ;{ }^{1} \mathrm{H}$ 
NMR (300 MHz, $\left.\mathrm{CDCl}_{3}\right) \delta$ 7.70-7.67 (m, 2H), 7.64-7.61 (m, 2H), 7.56-7.53 (m, 2H), 7.39-7.33 (m, 4H), 6.13 (s, 2H), $5.48(\mathrm{~s}, 1 \mathrm{H}), 3.78(\mathrm{~s}, 3 \mathrm{H}), 3.71(\mathrm{~s}, 6 \mathrm{H}), 3.46(\mathrm{~s}, 3 \mathrm{H}) ;{ }^{19} \mathrm{~F}$ $\left(282 \mathrm{MHz}, \mathrm{CDCl}_{3}\right) \delta-62.35:{ }^{13} \mathrm{C} \mathrm{NMR}\left(75 \mathrm{MHz}, \mathrm{CDCl}_{3}\right) \delta 170.2,162.6,160.7,141.3(\mathrm{q}$, $\left.{ }^{4} J(\mathrm{C}, \mathrm{F})=1 \mathrm{~Hz}\right), 137.0,130.4\left(\mathrm{q},{ }^{2} J(\mathrm{C}, \mathrm{F})=32 \mathrm{~Hz}\right), 128.2,128.0,127.8,126.7,124.9(\mathrm{q}$, $\left.{ }^{3} J(\mathrm{C}, \mathrm{F})=4 \mathrm{~Hz}\right), 124.2\left(\mathrm{q},{ }^{1} J(\mathrm{C}, \mathrm{F})=270 \mathrm{~Hz}\right), 106.1,100.6,90.8,89.5,87.2,55.6,55.3$, 52.1; HRMS: calcd for $\mathrm{C}_{27} \mathrm{H}_{25} \mathrm{~F}_{3} \mathrm{O}_{7}$ : 518.1552; found: $518.1554[\mathrm{M}+\mathrm{H}]^{+}$.

\section{Methyl}

$\left(2 S^{*}, 4 R^{*}, 5 S^{*}\right)-2-(2,4,6-$ trimethoxyphenyl)-4-phenyl-5-(4cyanomethylphenyl)-1,3-Dioxolane-4-carboxylate (4I): $\mathrm{mp}$ 152.0-152.6 ${ }^{\circ} \mathrm{C} ;{ }^{1} \mathrm{H}$ NMR $\left(300 \mathrm{MHz}, \mathrm{CDCl}_{3}\right) \delta$ 7.41-7.33 (m, 4H), 7.29-7.26 (m, 2H), 7.06-7.01 (m, 3H), $6.61(\mathrm{~s}$, 1H), 6.19 (s, 2H), $5.87(\mathrm{~s}, 1 \mathrm{H}), 3.88(\mathrm{~s}, 6 \mathrm{H}), 3.86(\mathrm{~s}, 3 \mathrm{H}), 3.85(\mathrm{~s}, 3 \mathrm{H}) ;{ }^{13} \mathrm{C}$ NMR $(75$ $\left.\mathrm{MHz}, \mathrm{CDCl}_{3}\right) \delta 173.6,163.1,161.3,142.9,136.0,131.3,128.7,127.9,127.5,126.2$, 119.0, 111.3, 102.4, 98.6, 90.9, 87.8, 84.3, 55.6, 55.5, 53.3; HRMS: calcd for $\mathrm{C}_{27} \mathrm{H}_{25} \mathrm{NO}_{7}$ : 475.1631; found: $475.1634[\mathrm{M}+\mathrm{H}]^{+}$. Single crystal was grown in hexane/ethyl acetate and corresponding X-ray structure was obtained.

\section{Methyl}

$\left(2 S^{*}, 4 R^{*}, 5 R^{*}\right)-2-(2,4,6-$ trimethoxyphenyl)-4-phenyl-5-(4cyanomethylphenyl)-1,3-Dioxolane-4-carboxylate (5I): $\mathrm{mp}$ 171.1-171.9 ${ }^{\circ} \mathrm{C} ;{ }^{1} \mathrm{H}$ NMR $\left(300 \mathrm{MHz}, \mathrm{CDCl}_{3}\right) \delta$ 7.68-7.63 (m, 4H), 7.52-7.50 (m, 2H), 7.40-7.36 (m, 3H), 7.30 (s, $1 \mathrm{H}), 6.14(\mathrm{~s}, 2 \mathrm{H}), 5.44(\mathrm{~s}, 1 \mathrm{H}), 3.82(\mathrm{~s}, 3 \mathrm{H}), 3.74(\mathrm{~s}, 6 \mathrm{H}), 3.51(\mathrm{~s}, 3 \mathrm{H}) ;{ }^{13} \mathrm{C}$ NMR $(75$ $\left.\mathrm{MHz}, \mathrm{CDCl}_{3}\right) \delta 170.0,162.7,160.7,142.6,136.6,131.8,128.5,128.2,128.0,126.6$, 118.9, 112.1, 105.8, 100.5, 90.9, 89.5, 87.0, 55.7, 55.4, 52.3; HRMS: calcd for $\mathrm{C}_{27} \mathrm{H}_{25} \mathrm{NO}_{7}$ : 475.1631; found: $475.1620[\mathrm{M}+\mathrm{H}]^{+}$. Single crystal was grown in hexane/ethyl acetate and corresponding X-ray structure was obtained. 
Methyl $\left(2 S^{*}, 4 R^{*}, 5 S^{*}\right)-2-(2,4,6-t r i m e t h o x y p h e n y l)-4-p h e n y l-5-(2,4-d i n i t r o p h e n y l)-$ 1,3-Dioxolane-4-carboxylate and Methyl $\left(2 S^{*}, 4 R^{*}, 5 R^{*}\right)-2-(2,4,6$-trimethoxyphenyl)4-phenyl-5-(2,4-dinitrophenyl)-1,3-Dioxolane-4-carboxylate $(\mathbf{4 m}+5 \mathrm{~m}) \quad$ (mixture of diastereomers): ${ }^{1} \mathrm{H}$ NMR $\left(300 \mathrm{MHz}, \mathrm{CDCl}_{3}\right) \delta(8.64(\mathrm{~d}, J=2.4 \mathrm{~Hz}, 1 \mathrm{H})$; minor diastereomer, 5m), 8.54 (m, 1H; major diastereomer, 4m), $(8.39$ (dd, $J=8.7,2.4 \mathrm{~Hz}$, $1 \mathrm{H})), 8.04(\mathrm{~m}, 2 \mathrm{H}),(7.94(\mathrm{~d}, J=8.7 \mathrm{~Hz}, 1 \mathrm{H})),(7.63-7.59(\mathrm{~m}, 2 \mathrm{H})), 7.39-7.30(\mathrm{~m}, 2 \mathrm{H})$, (7.39-7.32 (m, 3H)), (7.11 (s, 1H)), 7.05-7.02 (m, 3H), $6.75(\mathrm{~s}, 1 \mathrm{H}), 6.66(\mathrm{~s}, 1 \mathrm{H}),(6.25(\mathrm{~s}$, 1H)), $6.21(\mathrm{~s}, 2 \mathrm{H}),(6.16(\mathrm{~s}, 2 \mathrm{H})), 3.92(\mathrm{~s}, 6 \mathrm{H}), 3.91(\mathrm{~s}, 3 \mathrm{H}), 3.86(\mathrm{~s}, 3 \mathrm{H}),(3.84(\mathrm{~s}, 9 \mathrm{H}))$, (3.67 (s, 3H)); ${ }^{13} \mathrm{C}$ NMR (75 MHz, $\left.\mathrm{CDCl}_{3}\right) \delta 171.6(169.7), 163.3(163.0), 161.2(160.7)$, 148.2(148.8), 146.8(147.4), 140.8(138.3), 135.7(135.1), 132.8(131.4), 128.6(129.1), 127.7(128.3), 126.3(125.97), 125.89(126.03), 119.0(120.2), 100.3(105.1), 99.1(101.9), 91.0(90.9), 88.3(89.8), 79.3(82.8), 55.6(55.8), 55.5(55.4), 53.4(52.8) ; HRMS: calcd for $\mathrm{C}_{26} \mathrm{H}_{24} \mathrm{~N}_{2} \mathrm{O}_{11}$ : 540.1380; found: $540.1385[\mathrm{M}+\mathrm{H}]^{+}$.

Procedure for Reaction of Phenyldiazoacetate with 2,4-dinitrobenzaldehyde: To a 8 $\mathrm{mL} \mathrm{CH}_{2} \mathrm{Cl}_{2}$ solution of $\mathrm{Rh}_{2}(\mathrm{OAc})_{4}$ (4.4 mg, $\left.0.01 \mathrm{mmol}\right)$, 2,4-dinitrobenzaldehyde(196 mg, $1.0 \mathrm{mmol}$ ) was added methyl phenyl diazoacetate (176 mg, $1.0 \mathrm{mmol})$ in $4 \mathrm{~mL}$ of $\mathrm{CH}_{2} \mathrm{Cl}_{2}$ via a syringe pump over $1 \mathrm{~h}$ under refluxing. After completed addition, the reaction mixture was cooled to room temperature. Solvent was removed and a portion of crude product was subjected to ${ }^{1} \mathrm{H}$ NMR analysis for determination of the isomer ratio. Only one stereoisomer was observed. The crude product was purified by flash chromatography on silica gel by using petroleum ether:EtOAc $=10: 1$ as eluent to give stereospecific epoxide $196 \mathrm{mg}$ (57\% yield, white powder). 


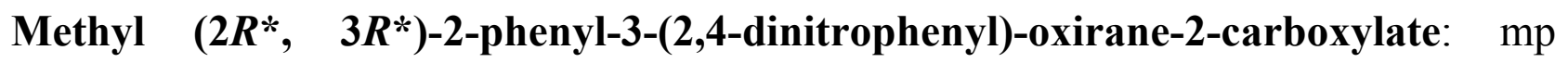
152.4-153.7 ${ }^{\circ} \mathrm{C} ;{ }^{1} \mathrm{H}$ NMR $\left(300 \mathrm{MHz}, \mathrm{CDCl}_{3}\right) \delta 9.05(\mathrm{~d}, J=2.4 \mathrm{~Hz}, 1 \mathrm{H}), 8.55(\mathrm{dd}, J=8.7$, $2.4 \mathrm{~Hz}, 1 \mathrm{H}), 8.09(\mathrm{~d}, J=8.7 \mathrm{~Hz}, 1 \mathrm{H}), 7.74-7.69(\mathrm{~m}, 2 \mathrm{H}), 7.50-7.43(\mathrm{~m}, 3 \mathrm{H}), 4.76(\mathrm{~s}, 1 \mathrm{H})$, $3.48(\mathrm{~s}, 3 \mathrm{H}) ;{ }^{13} \mathrm{C}$ NMR $\left(75 \mathrm{MHz}, \mathrm{CDCl}_{3}\right) \delta 166.6,148.0,147.5,137.2,133.5,131.2$, 129.5, 128.8, 128.2, 127.0, 120.3, 66.8, 63.0, 53.0; HRMS: calcd for $\mathrm{C}_{16} \mathrm{H}_{12} \mathrm{~N}_{2} \mathrm{O}_{7}$ : 344.0645; found: $344.0644[\mathrm{M}+\mathrm{H}]^{+}$. 
$\mathrm{X}$-ray structure of $\mathbf{4 b}$

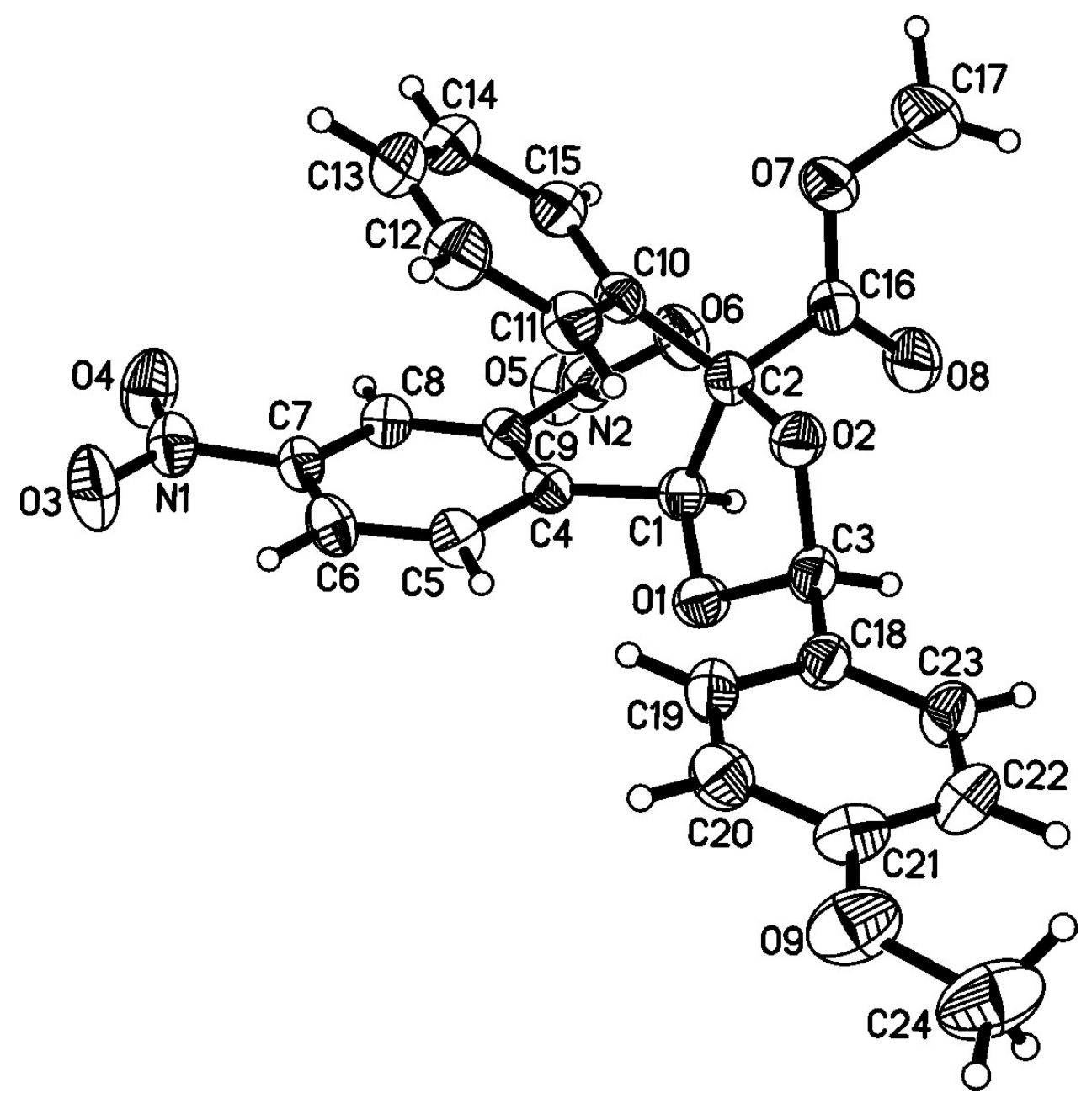


EEOOD $0-$

OESSE I-

8L0:9 1

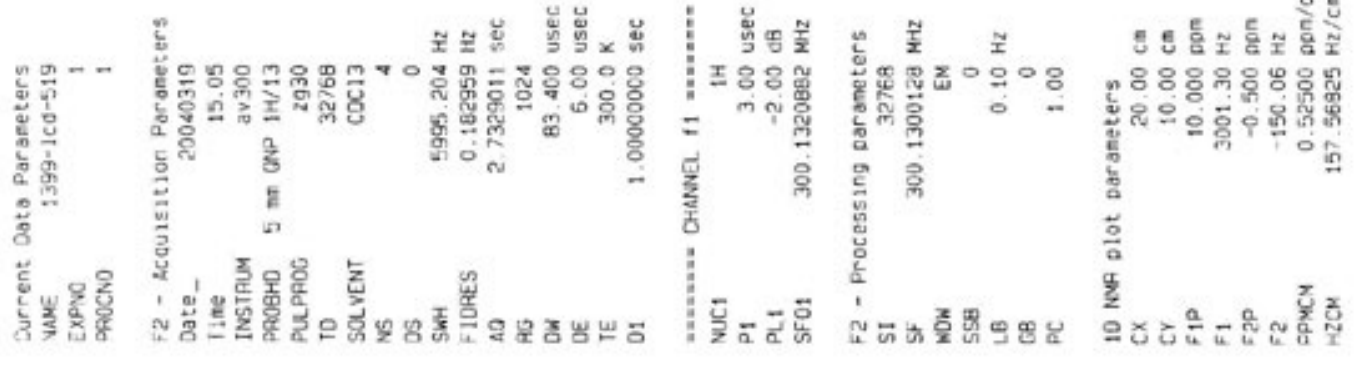

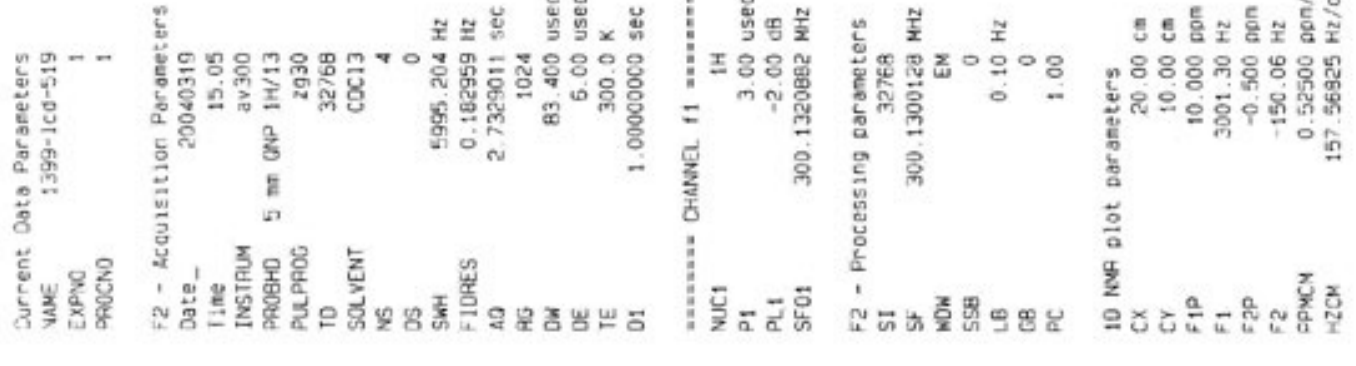

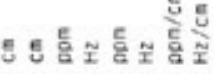

$20 G_{L} / \varepsilon^{\circ} \mathrm{E}-$

06068 ' $\mathrm{E}->$

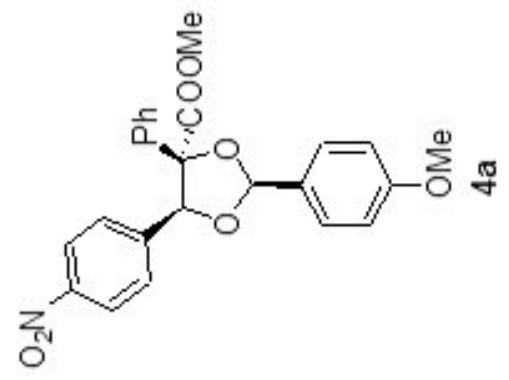

$\angle \angle: 000^{\circ}$

968EL' 9

gorlico 2

$\bar{m} \varepsilon \nabla L 20^{\circ} L$

ปั $90050^{\circ} \mathrm{L}$

${ }^{3} \varepsilon 9 \angle 90^{\circ} \mathrm{L}$

$19 \varepsilon \angle 0^{\circ}<-$

$89280^{\circ} \mathrm{C}$

$\angle 9 t 1 L^{\circ} \angle$

गटाटा 2

GटLCI' $L$

$\triangle \angle L E I L$

DW8G2 $L$

EggeE $L$

$\$ 6 / 9 \varepsilon^{\circ} L-$

$\left[61 \angle 9^{\circ} L\right]$

$59769^{\circ} L$

5.9516 $L$

g0126 ' $L$

$997 v 6:$

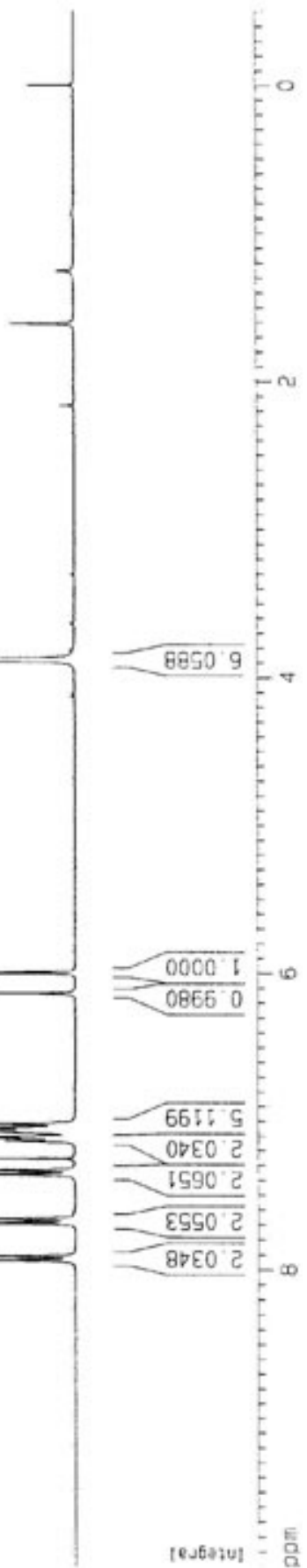




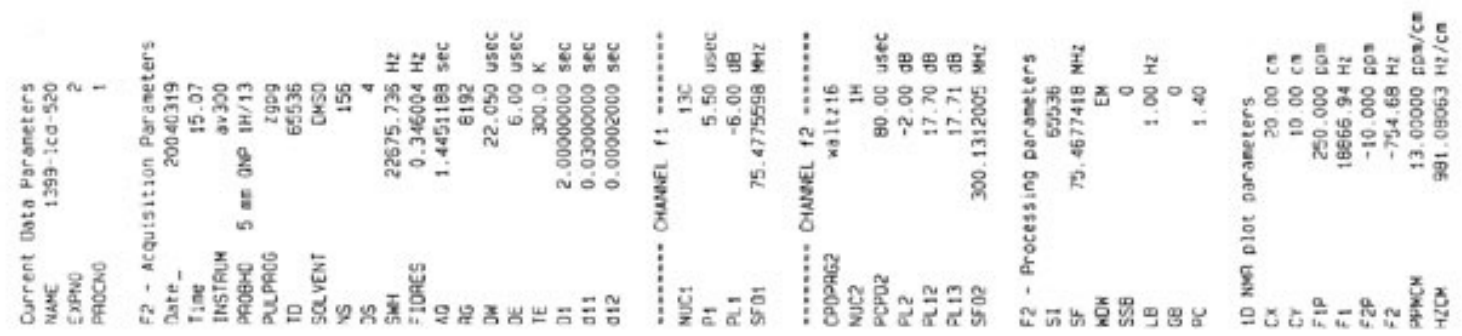

$687^{\circ} \mathrm{ES}$

SIS.gS

$25 t^{\circ} 9 L$

$5 L T<L$

$659^{\circ} \angle$

टБट $\nabla 8$

ग6G' 88

$998 \cdot \mathrm{EOt}$

द9เ $7 \mathrm{LI}$

606 टटा

टा8 दटा

$010<21$

560 вट

Gट० बटा

टBG बटा-

EG9 8टा

6LC'GE

OLC ED

$89 D^{\circ} \angle D I=$

E $\angle 0^{\circ}: 9 t$

DAS $2 \angle 1$
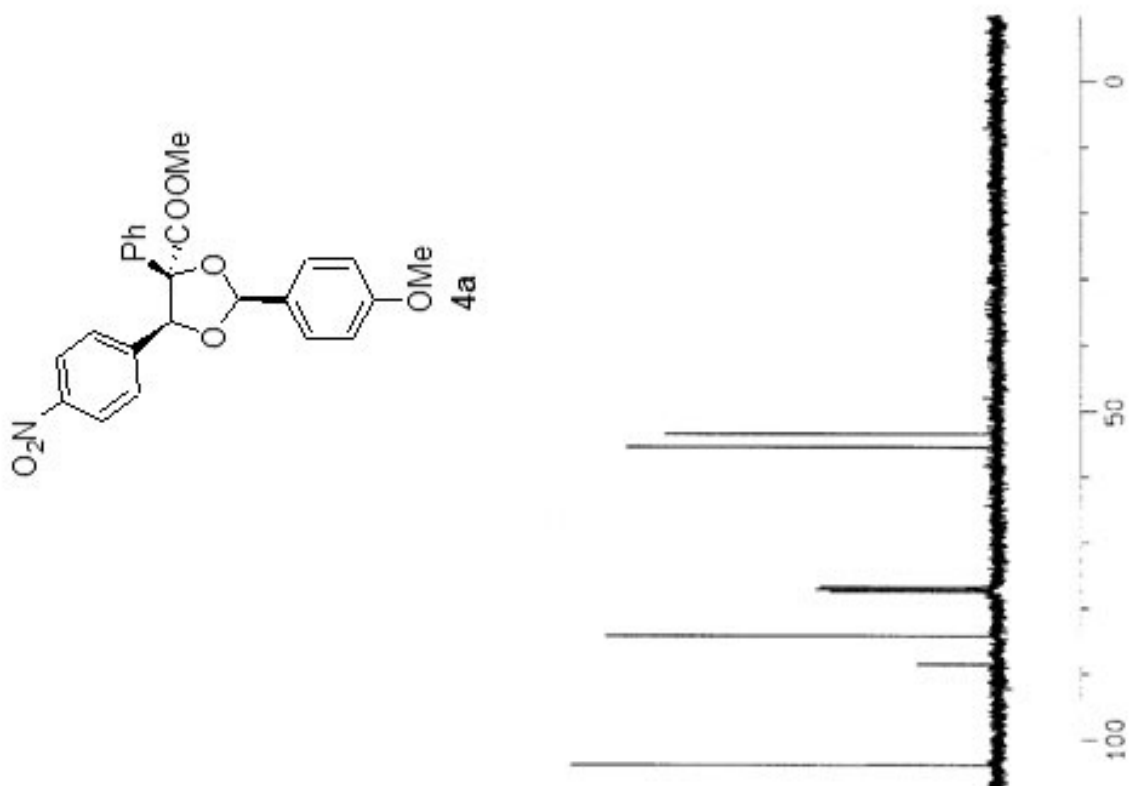

$-\stackrel{8}{9}$

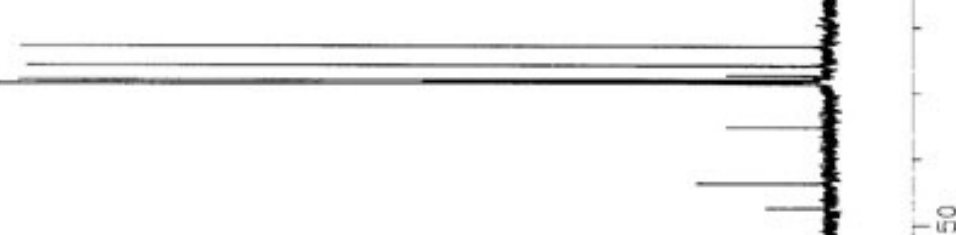

-온

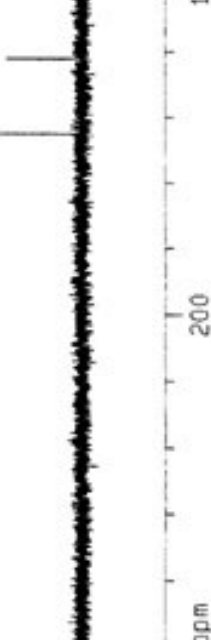



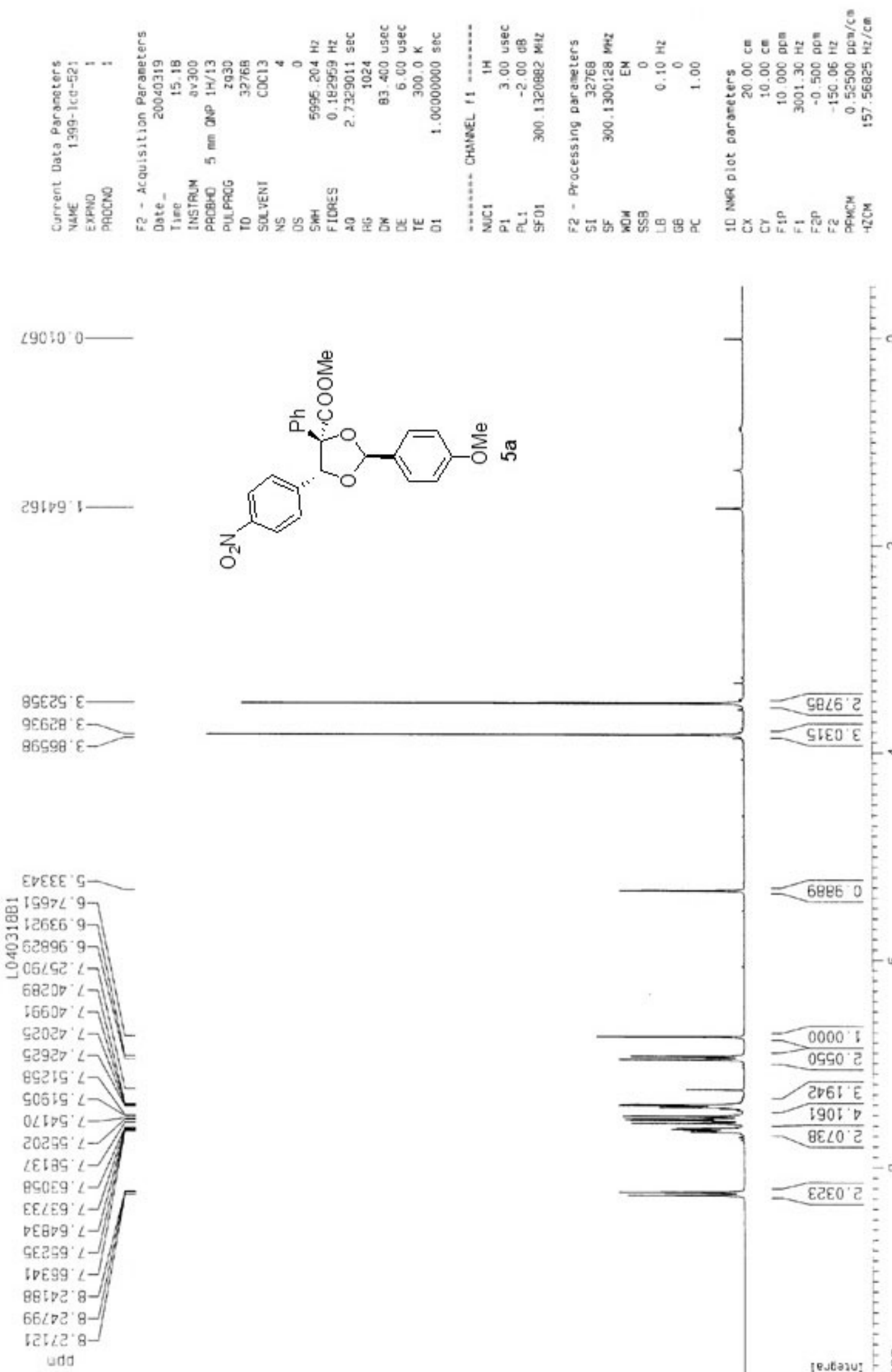

8รEटद $\varepsilon$

$95620^{2} \mathrm{8}-2$

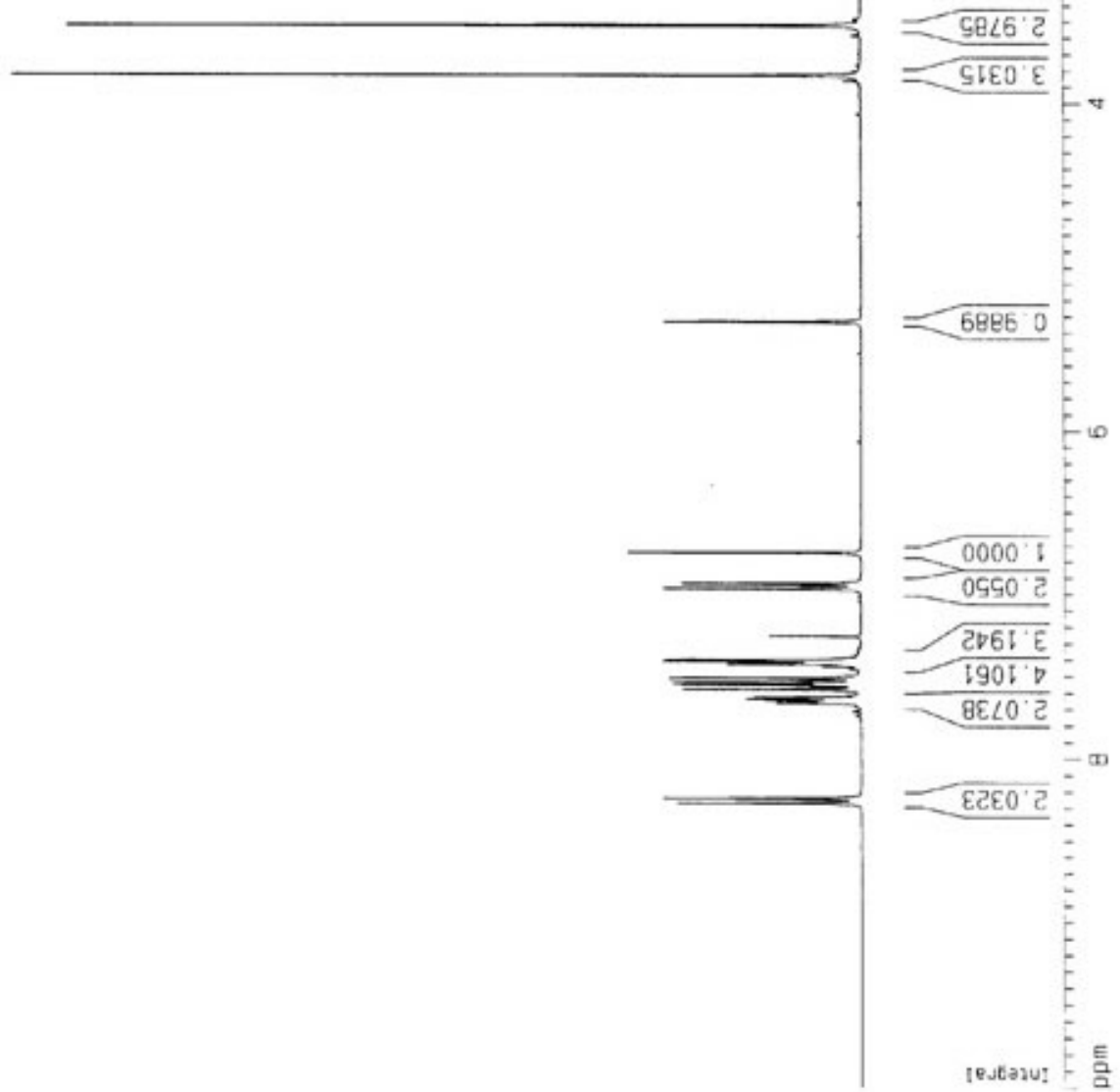




$$
r
$$



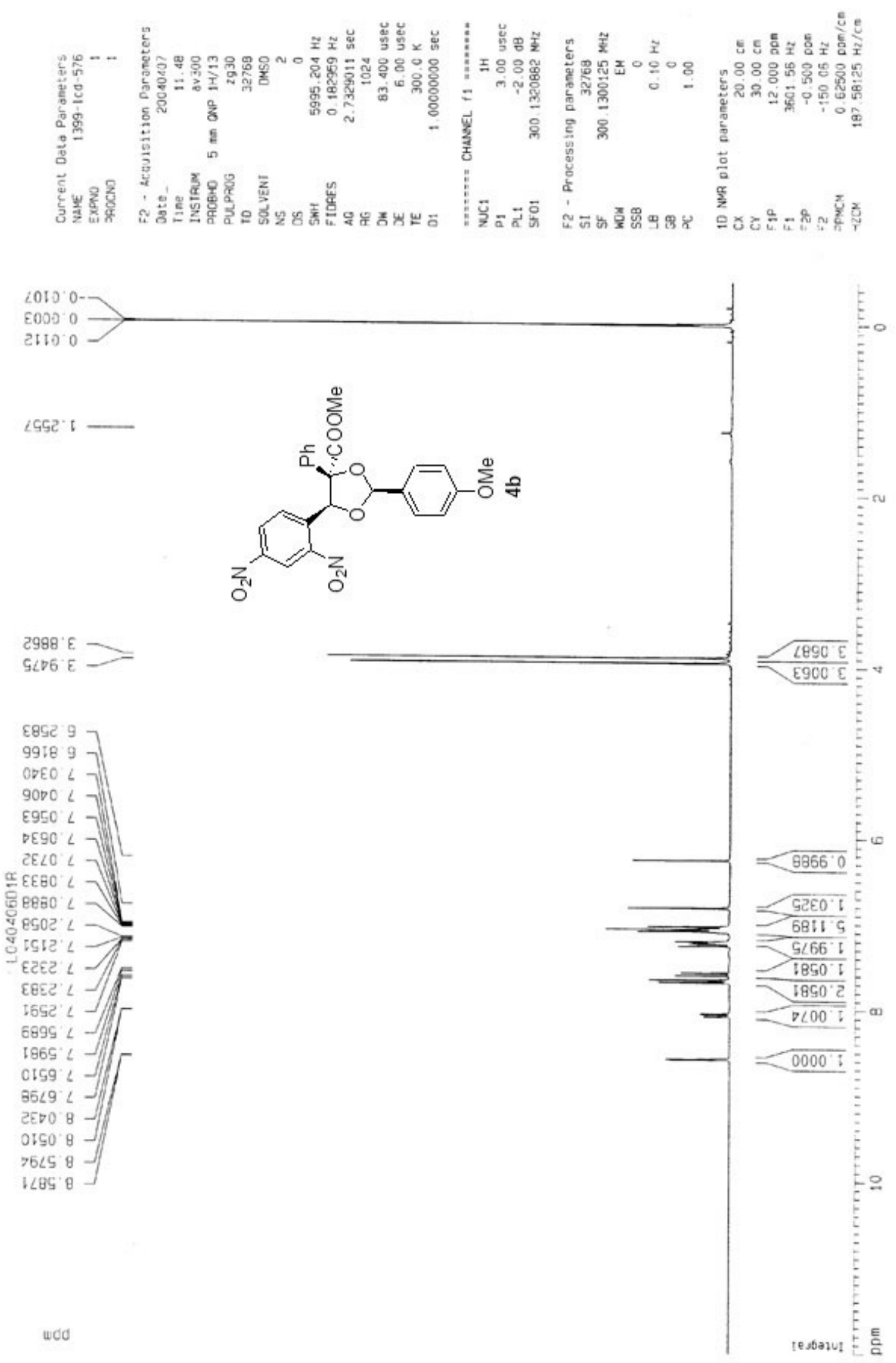


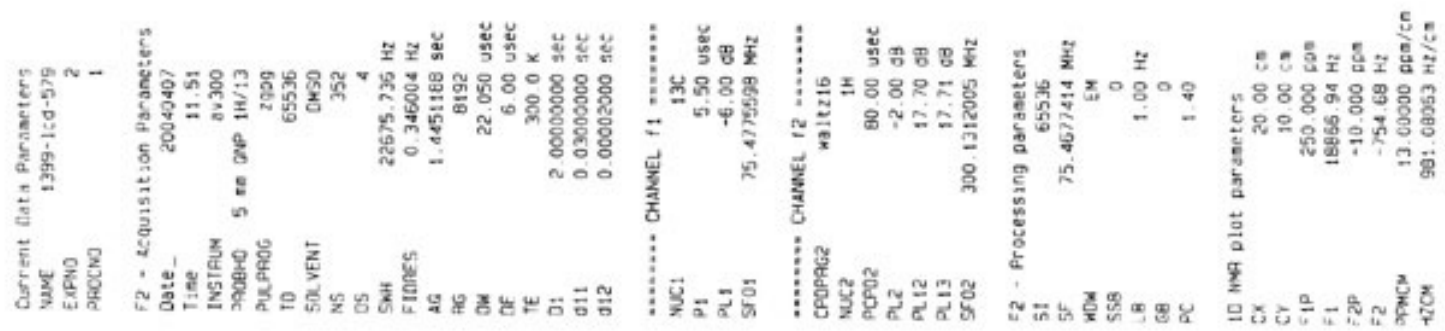

689 ES

จ21 0

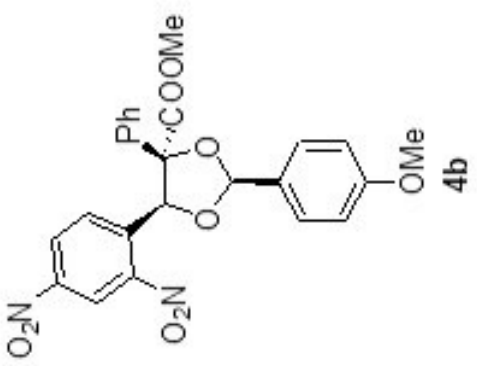

$\nabla E L / 9 L$

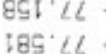

$58 \angle 6 L$

Egा 68

$590 \div 017$

$56 \mathrm{C}^{\circ} \nabla \mathrm{LI}-$

$\angle 80^{\circ} 611-$

ISD 925

टG0 $\angle 21-$

G9I ' $82:-$

$66 \varepsilon$ ' $82:$

$\checkmark 10$ टE:

285 $\triangle E \mathrm{~L}-$

$996^{\circ} 6 \mathrm{EL}-$

$950^{\circ} \mathrm{CDI}-$

055 e०I-

[D: $191-$

$120 \quad 1 \angle 1$
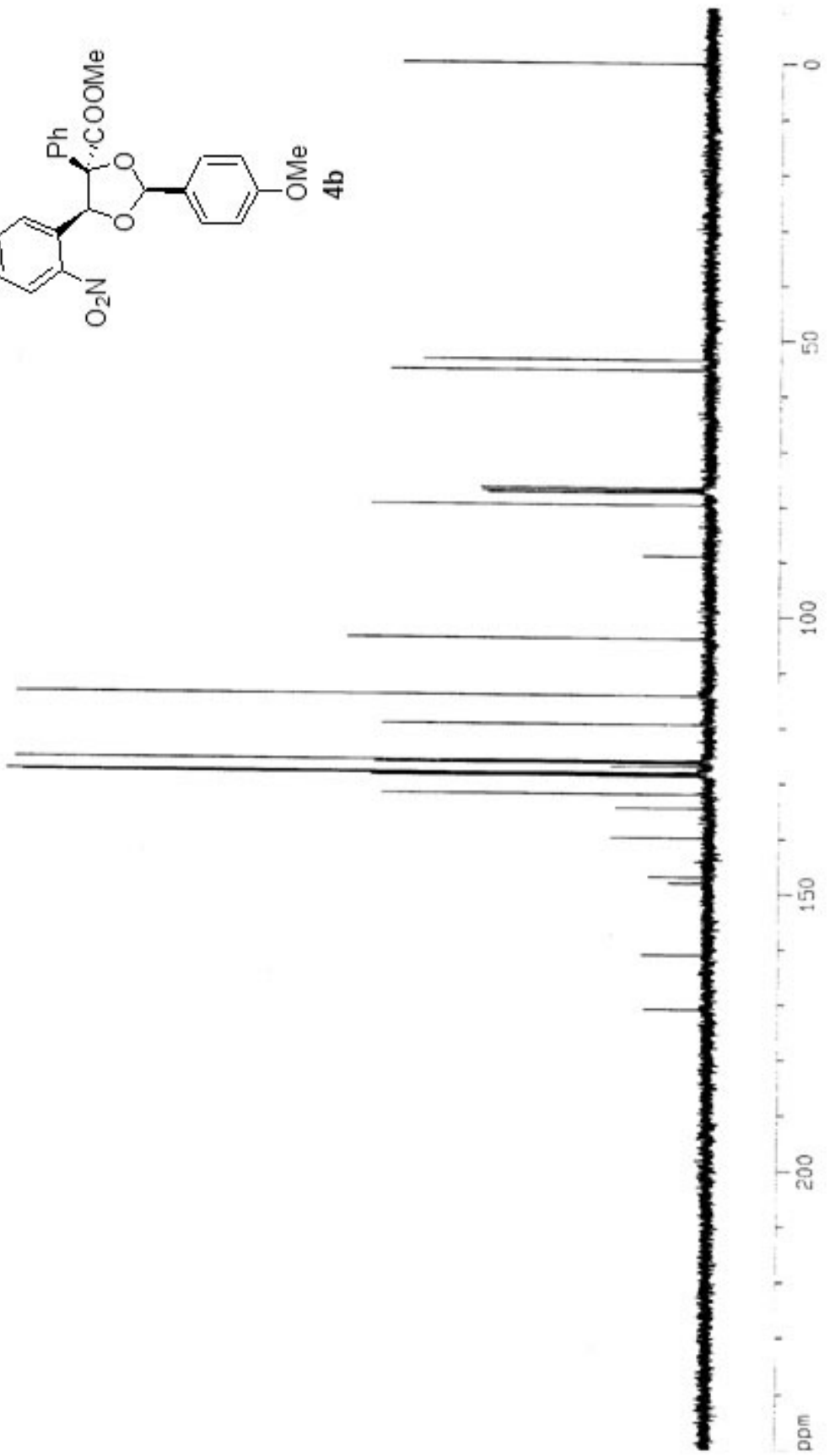

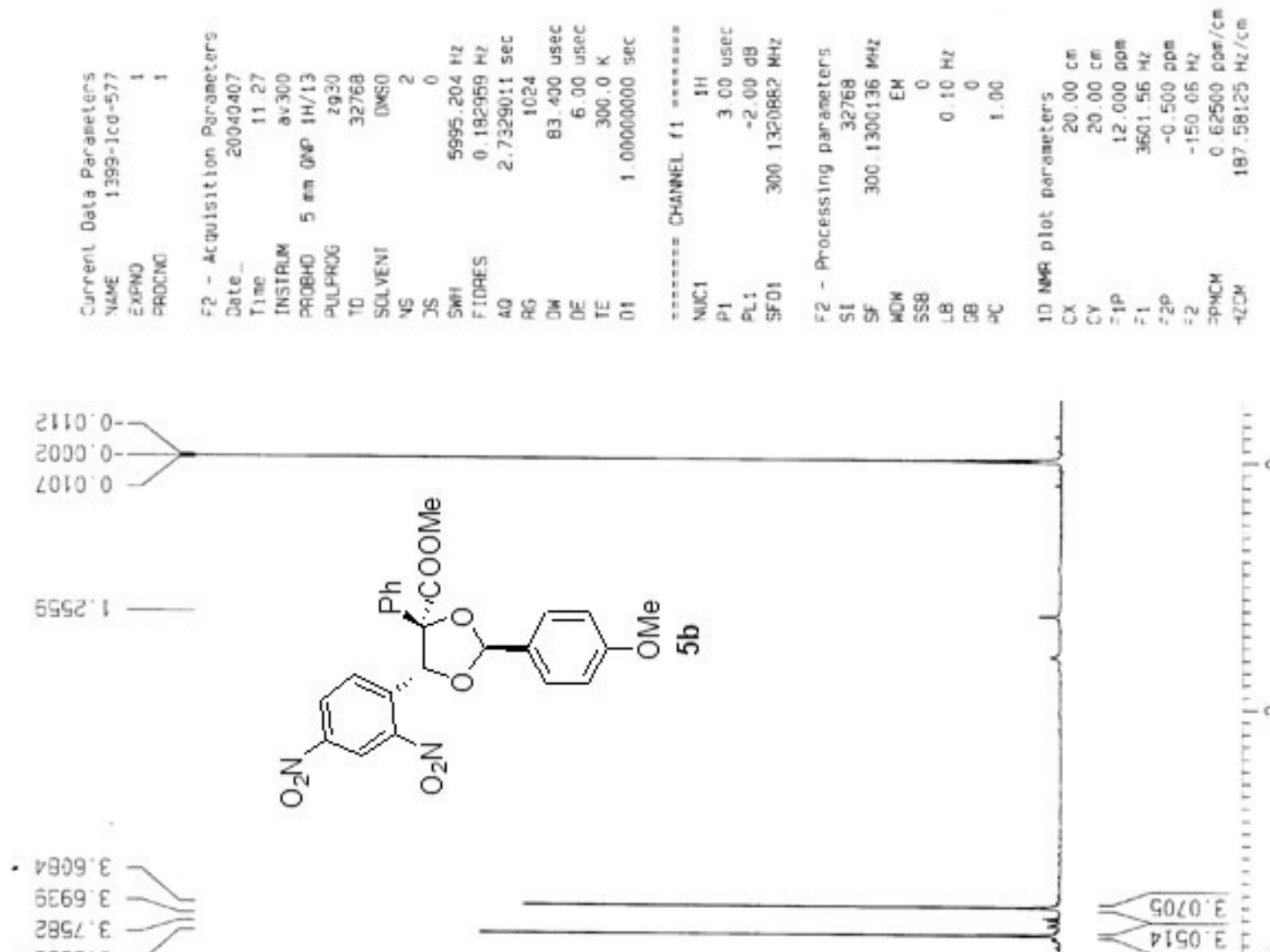

- gge日 E
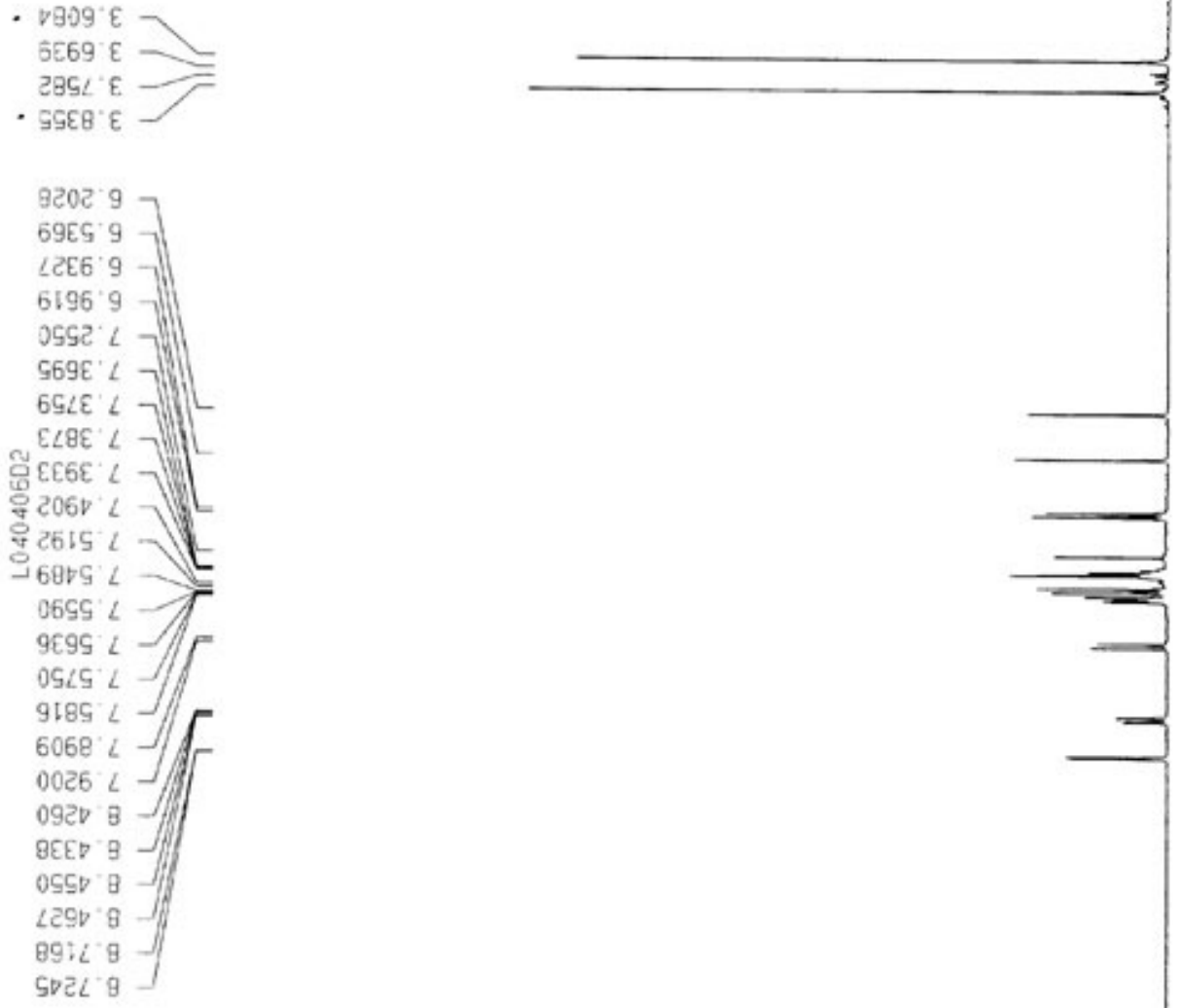

$=90 \angle 0^{\circ} \mathrm{E}$

$=2190^{\circ}$ 


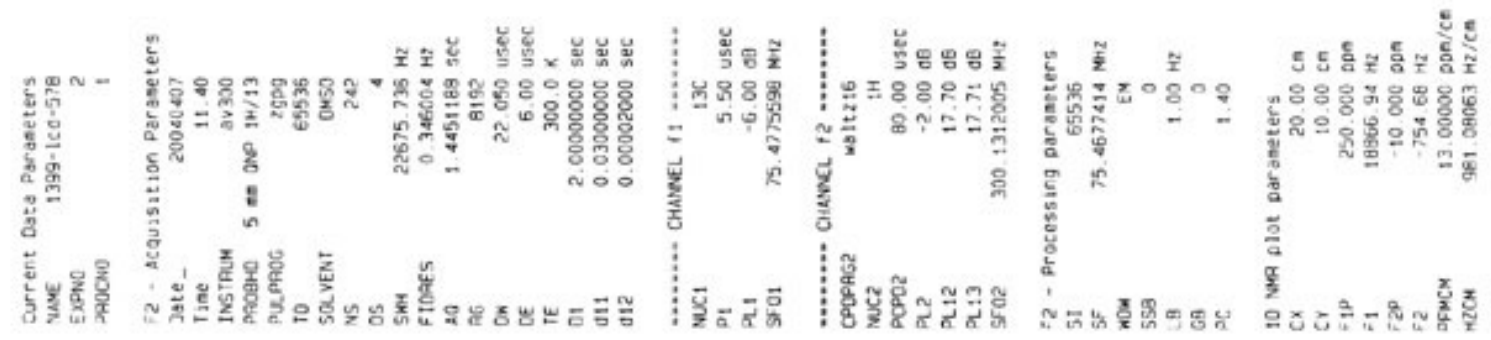

OEL 0

$186 \cdot 25-$
$960.55-$

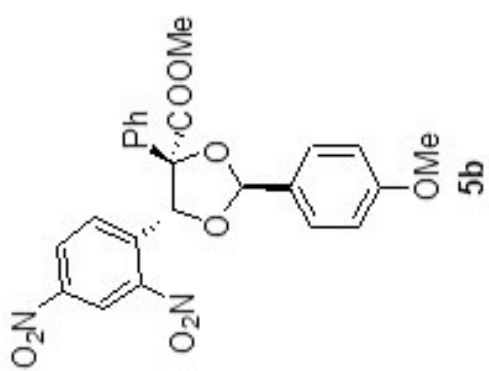

$56 L^{\circ} 9 L$
$891^{\circ}<L$

O 10.8

ह60 $06 \ldots$

टह0. 901

501 $\nabla 11$

$\angle 20021$

हCO $95 \mathrm{I}$

$\angle 99921$

992.821

vट5. 821

등

टEV 621-

6OL OEI $/$

: $\angle 9$ SEI -

5VE $8 \mathrm{LI}$

$\angle 59^{\circ} \angle O I-$

$0<9^{\circ} 801-$

$\angle \nabla 6^{\circ} 091-$

902691 

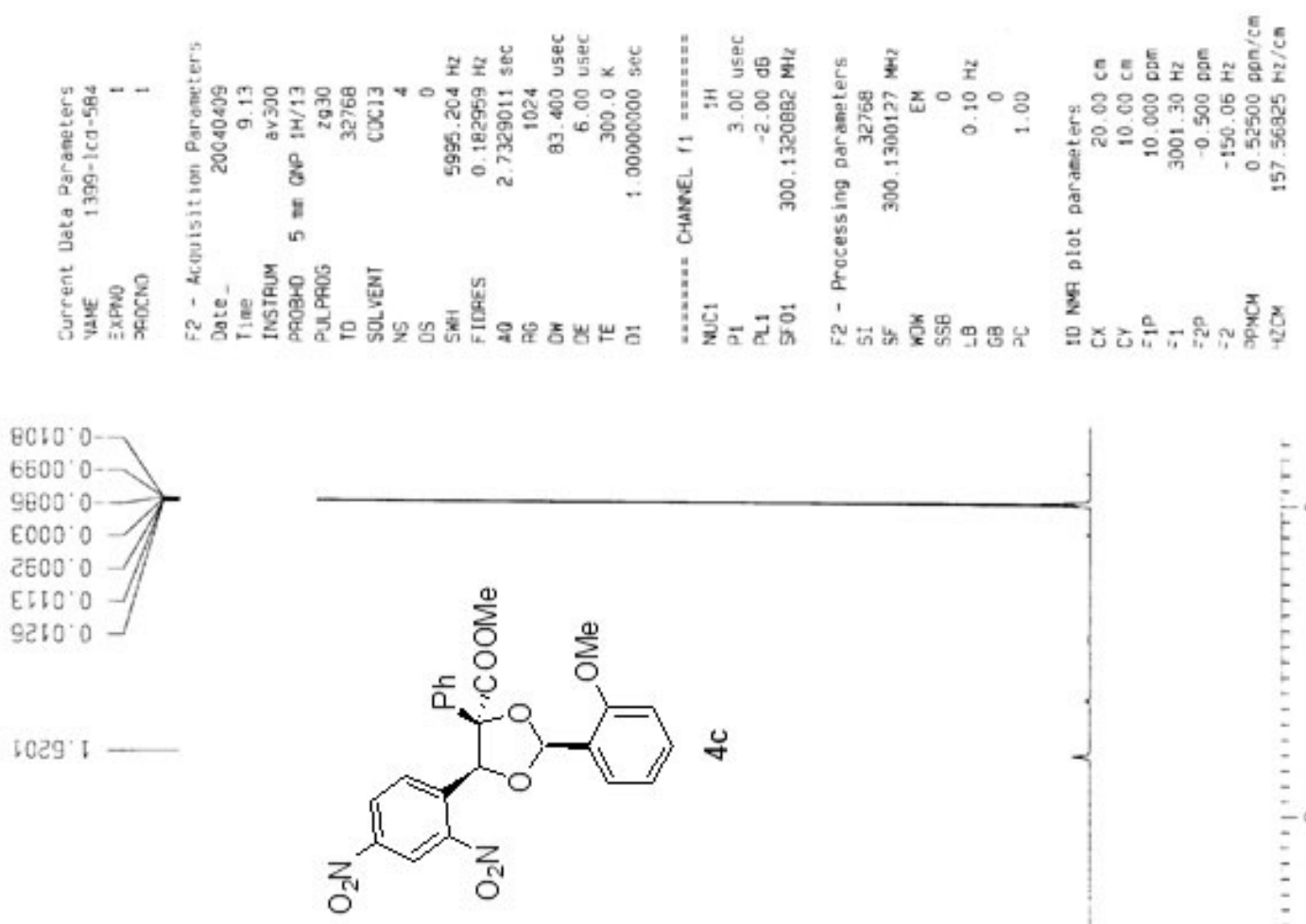

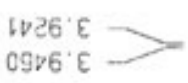
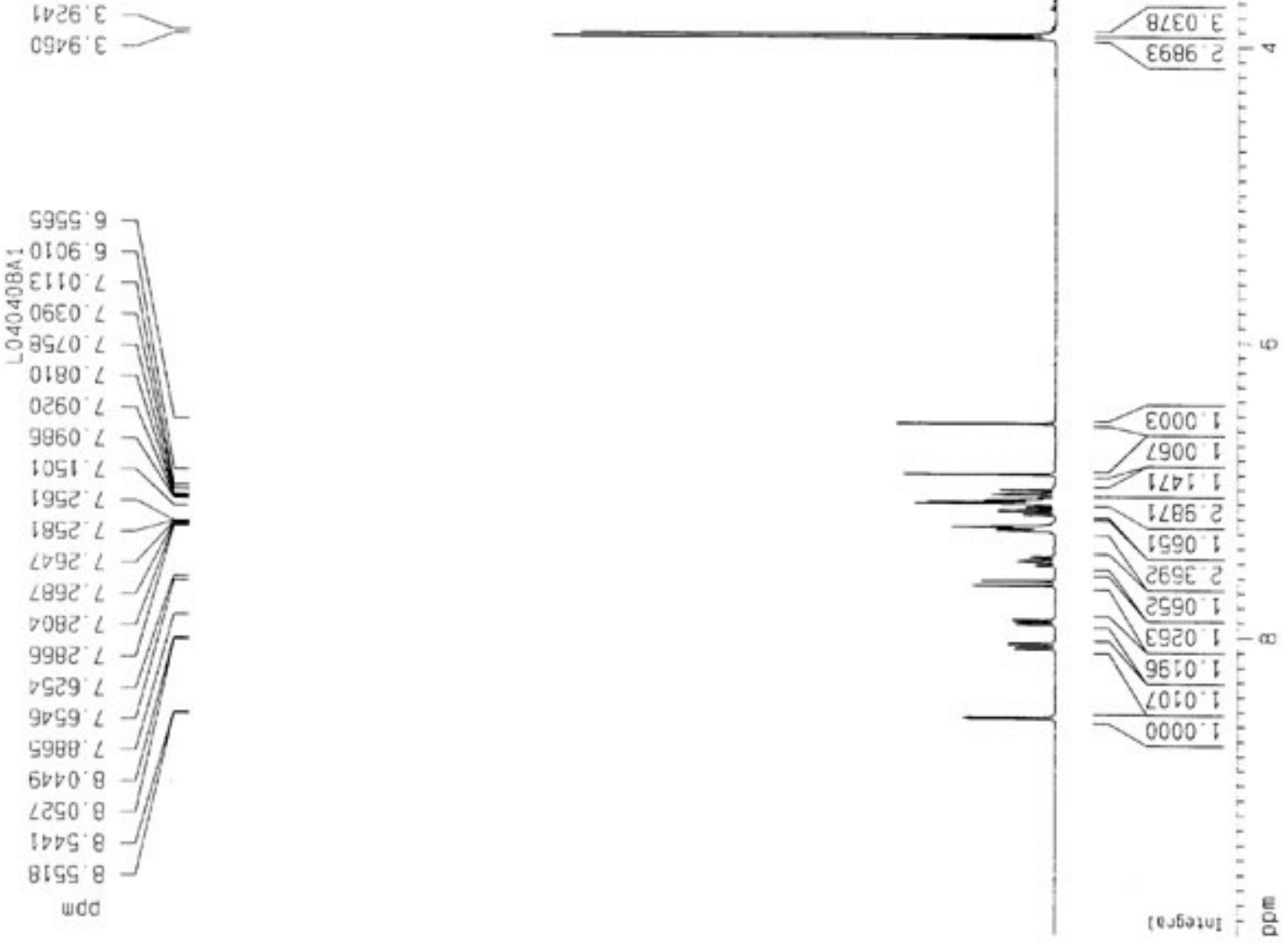


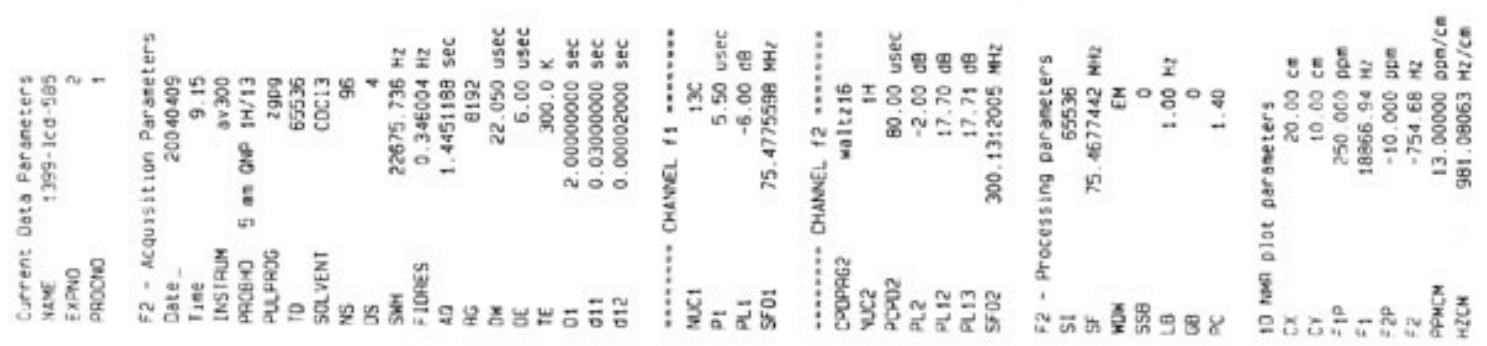

LOT'O

$\angle 99$ ' -

998 ' ५

$8 \varepsilon L \cdot 9 L$

191 $\angle L$

tge $\angle L$

ร8ऽ $\angle L$

IVG $6 L$

TLI 68

D $10.00 \mathrm{D}-$

DEE

606515

8LL OLI -

LLL टरा

000921

द.8 $921-$

950 LCI-

$1 \angle \mathrm{I} 8 \mathrm{~B} \mathrm{I}$

EVL BCI -

EES IE: - DEs

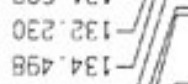

ICE $O D:-/ /$

$656 \cdot 9 \mathrm{bI}-$

$590.80 \mathrm{~L}-$

ग0ट $891-$

G66.0L:
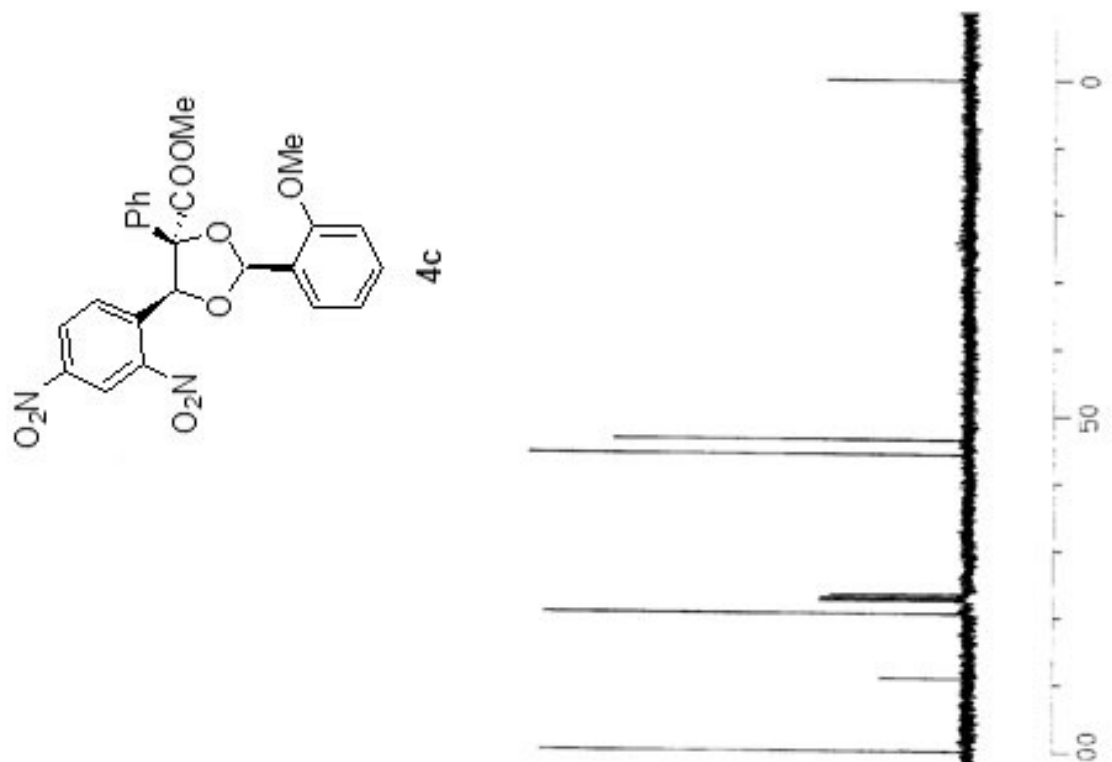


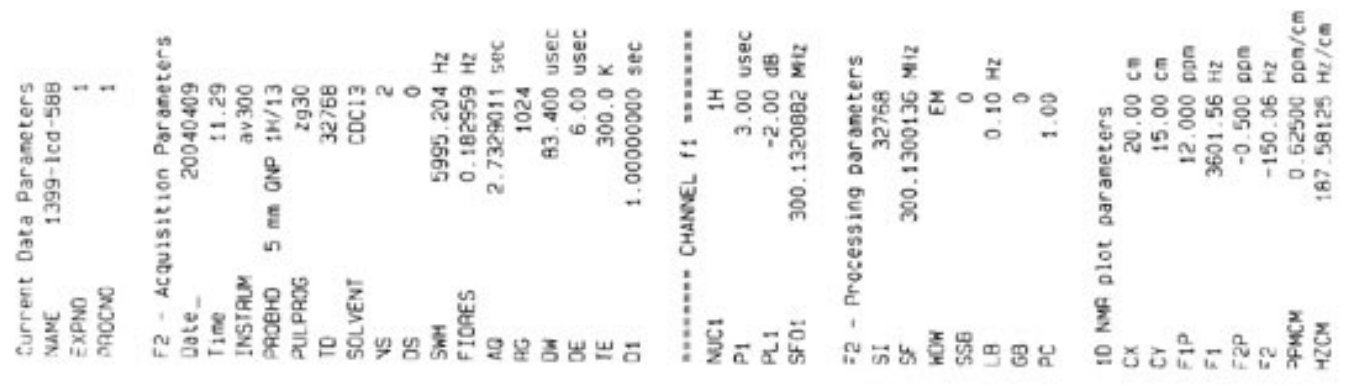

$\varepsilon 000^{\circ} 0$

DOto 0

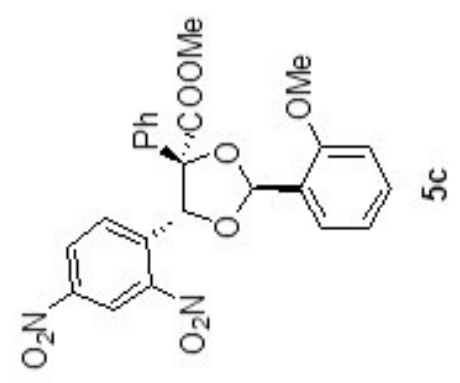

8909 $\varepsilon$

टाह6 $\mathrm{E}$

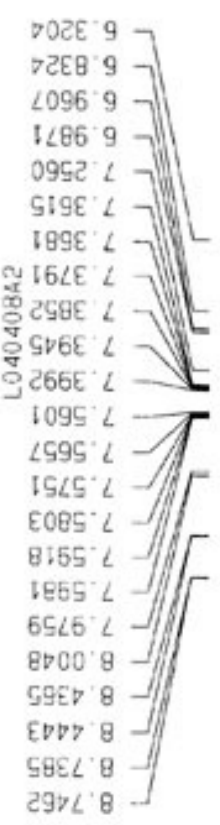

. 


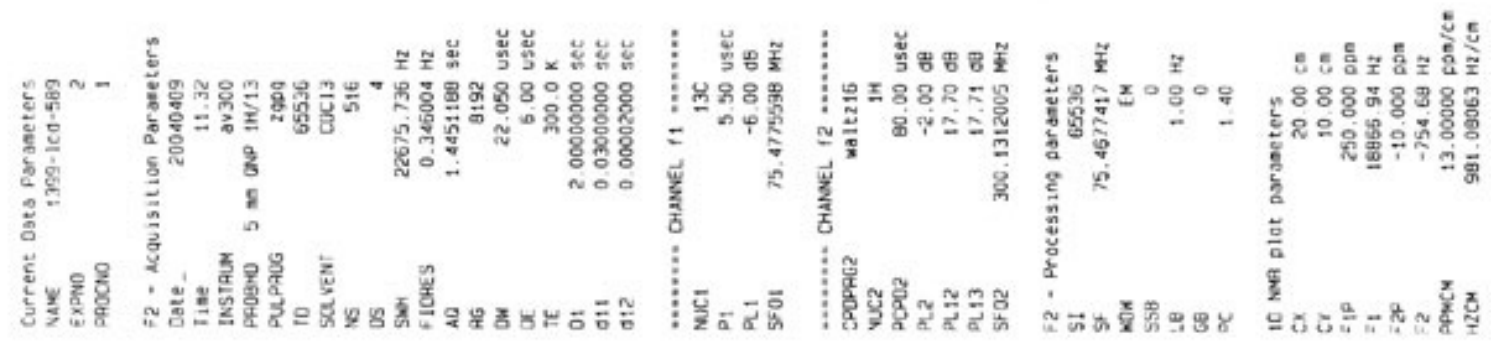

82:0<smiles>N#[C+]c1cc([W]=O)ccc1[C@@H]1OC(c2ccccc2[O-])OC1([O-])[O-]</smiles>

$6 E 6.25-$
$682.55-$

SEL $9 L$

$895 \cdot L L$

$\angle S E ' \angle L$

$285^{\circ} \angle L$

$598 \cdot 28$

It: 06

ह9: $20:$

$\varepsilon 80$. H.

६५દ O्ट

टह9 ०ट:

จह9 $\square ट 5$

$080.92:$

$929.921-$

$108^{\circ} \angle 25-$

५08' $82:-$

ดटЕ' 62:-

908 Oह:- -

ยटE' เE: $-/ / F$

จट6 डe:-

$9 \nabla 8$ 8E:-

I6I $\angle \mathrm{VI}-$

$8028 \mathrm{i}:$

ธะ६ ' $\angle$ G: -

992 '69:-

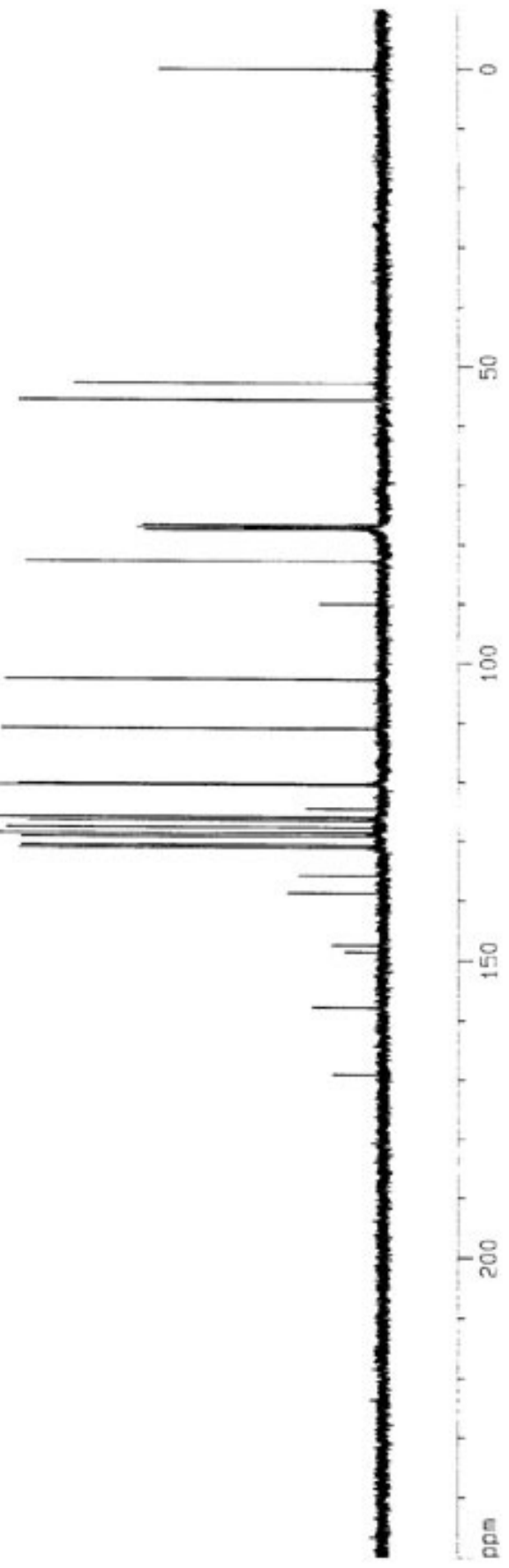



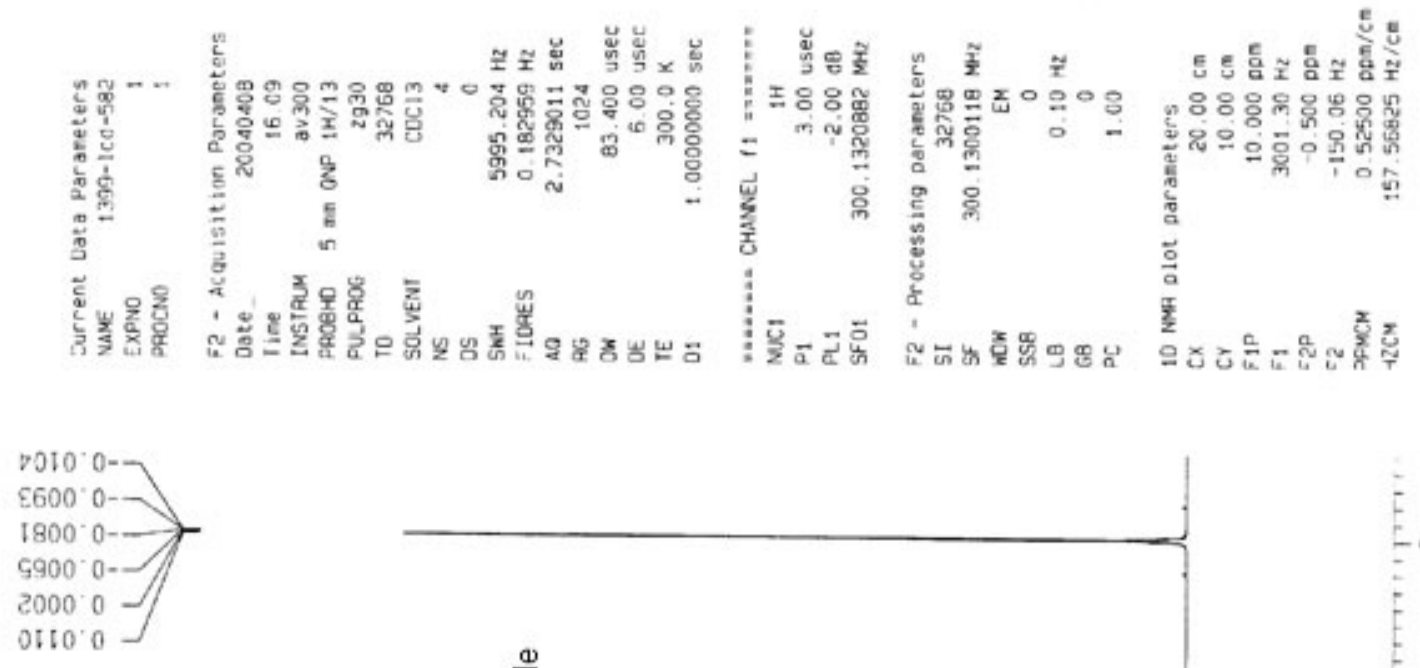

9992: ?

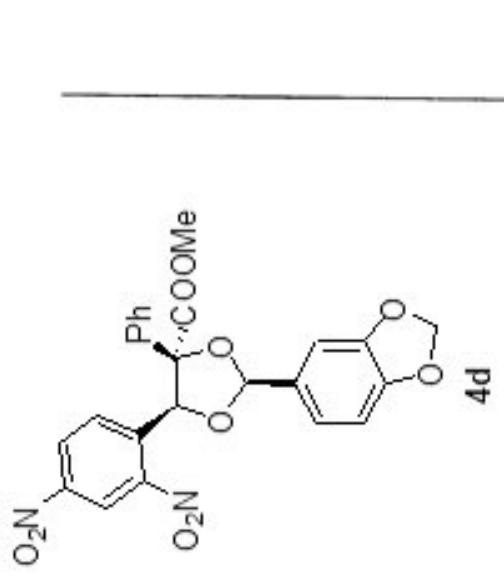

เEE ' $\varepsilon$
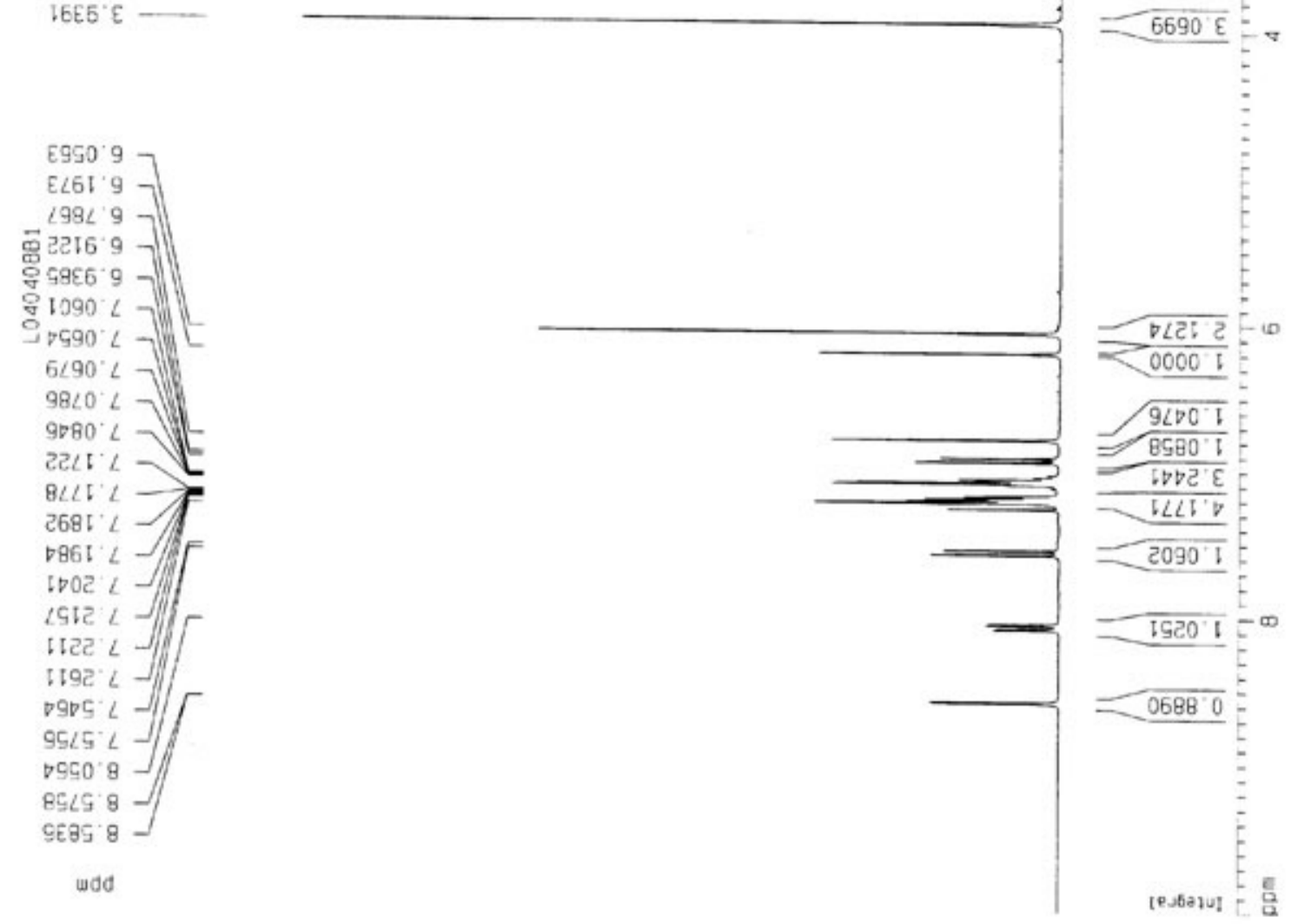


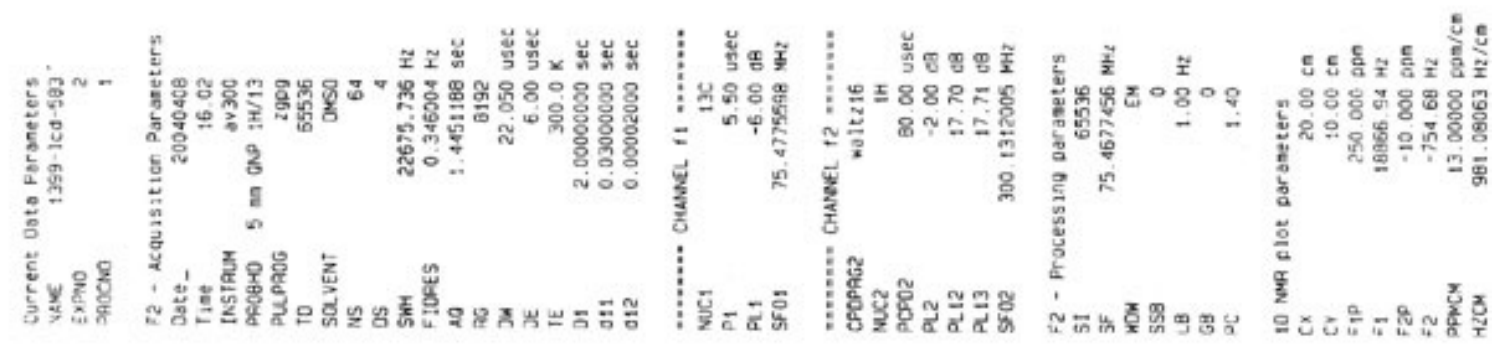

5800

899 59

DCL $9 L$

6ot $\angle L$

टLS $U L$

दEO 58

829101

จ०

$800 \angle 01$

oDS 801

हणะ Iट!

$\angle 00$ ' 21

$\angle 97.921$

मटा $82 \mathrm{t}$

$929 \cdot 851$

5EL $851-1 /$

EB8 IEI $-1 /$

SED $\nabla E I-1$

BEL $6 E !-$

$850 \angle D I-$

$5 \angle 0.801-$

$601281-$

26 I $^{\circ} 67 \mathrm{I}$

$898.0<1-$

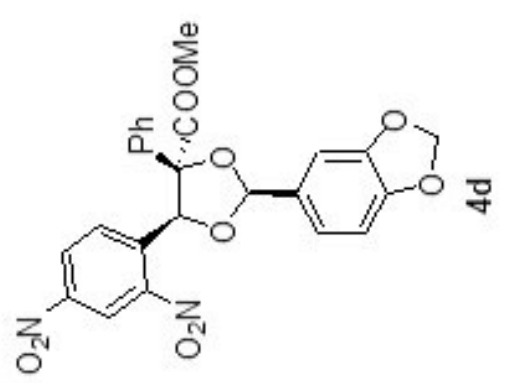

뭄

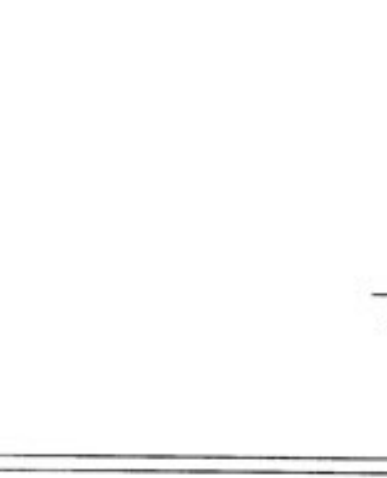



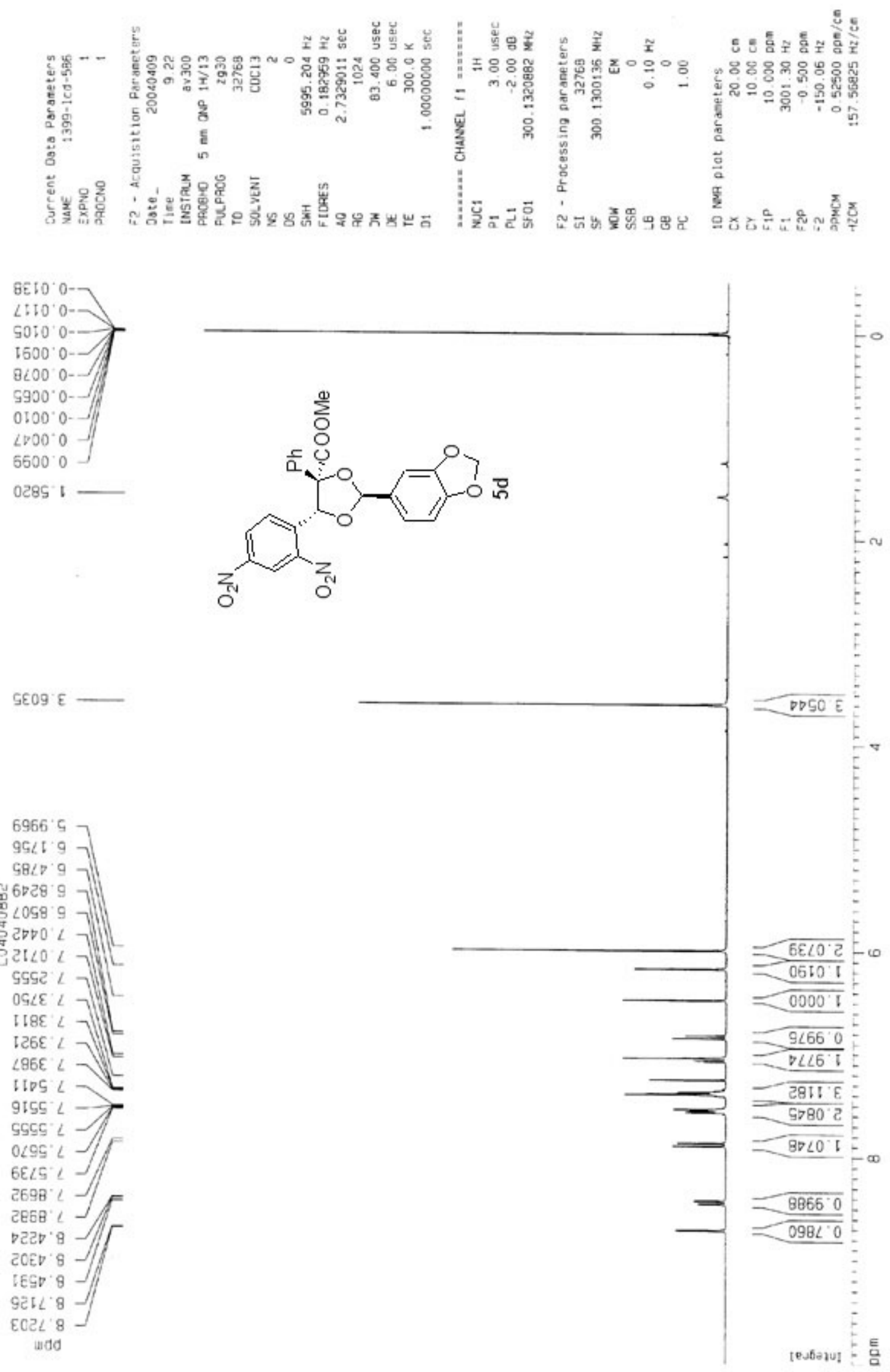


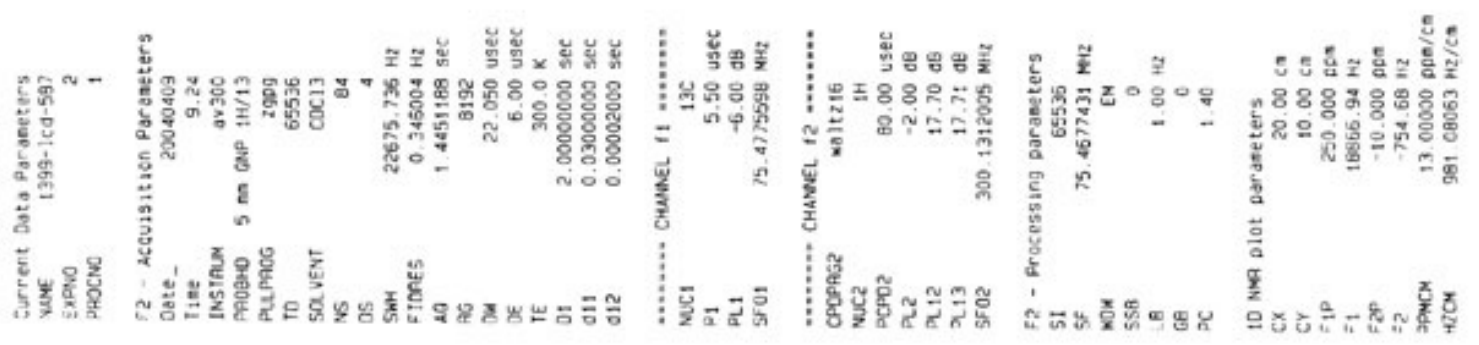

टाเ 0

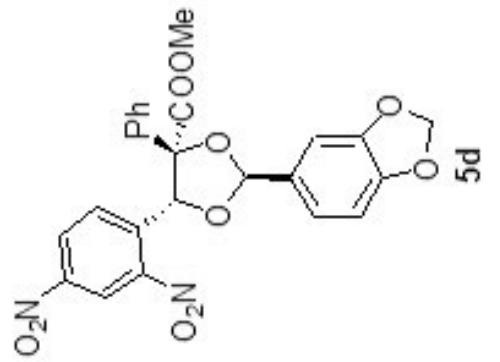

$986.25-$

O

टEL 9

9S: $\angle L$

$6 \angle 5 \angle L$

टट0 89

$\checkmark \angle O^{\circ}{ }^{\circ}$

DCS 101

हृत् 501

$6 \angle 8 \cdot 901$

SOE 80

ger $0{ }^{2}$

टहा Iए।

: $50.521-$

$855.921-$

BE, Bटा

59० $651-1$

OL $\angle$ OEL -

EEB OEL $1 / / F$

LEO SE! -

BDI' $8 E[-$

$999^{\circ} \mathrm{CDI}-$

$060^{\circ} 801-$

Itg. $80 \mathrm{DI}-$

$800601]$

$060.691-$ 

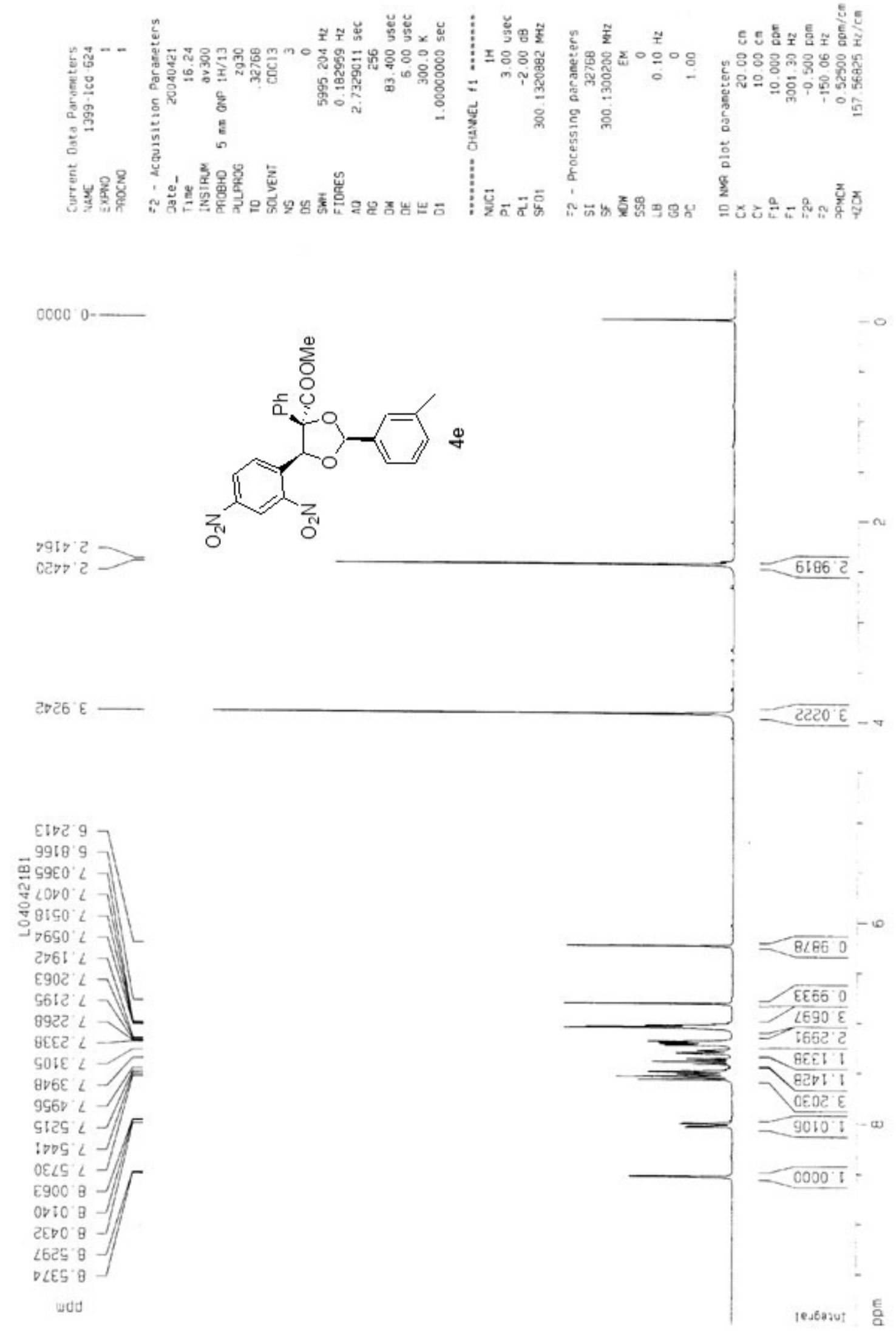


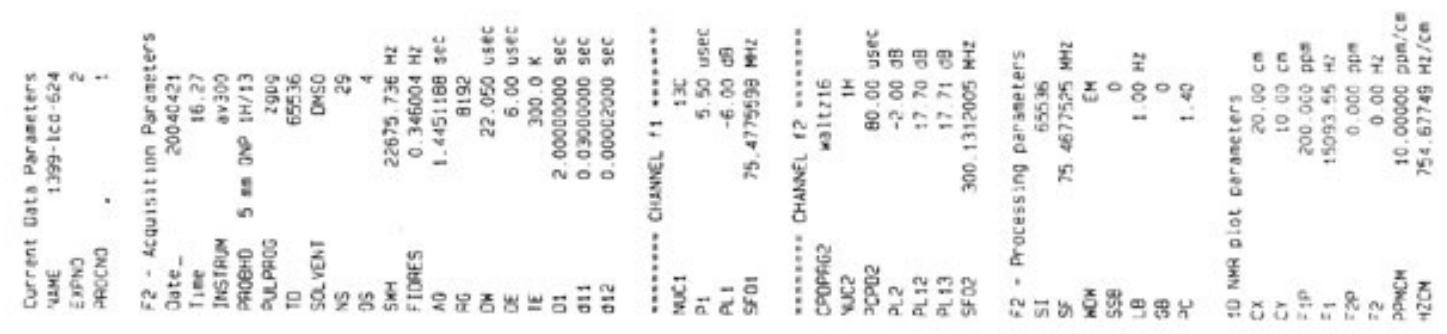

609 : 2

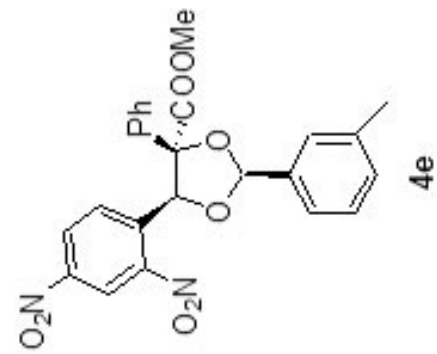

LCE ES

Z

$D E L 9 L$

ogt $\angle L$

हยS $\measuredangle$

$\angle 896 L$

901 68

806.50

ह5ะ 6117

ट85 Eटा

홈

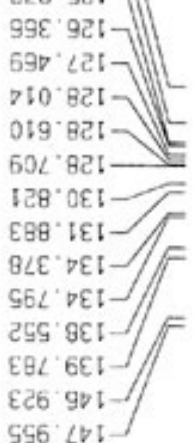

दc6. $\angle 4:$

ट28 $0 \angle:-$

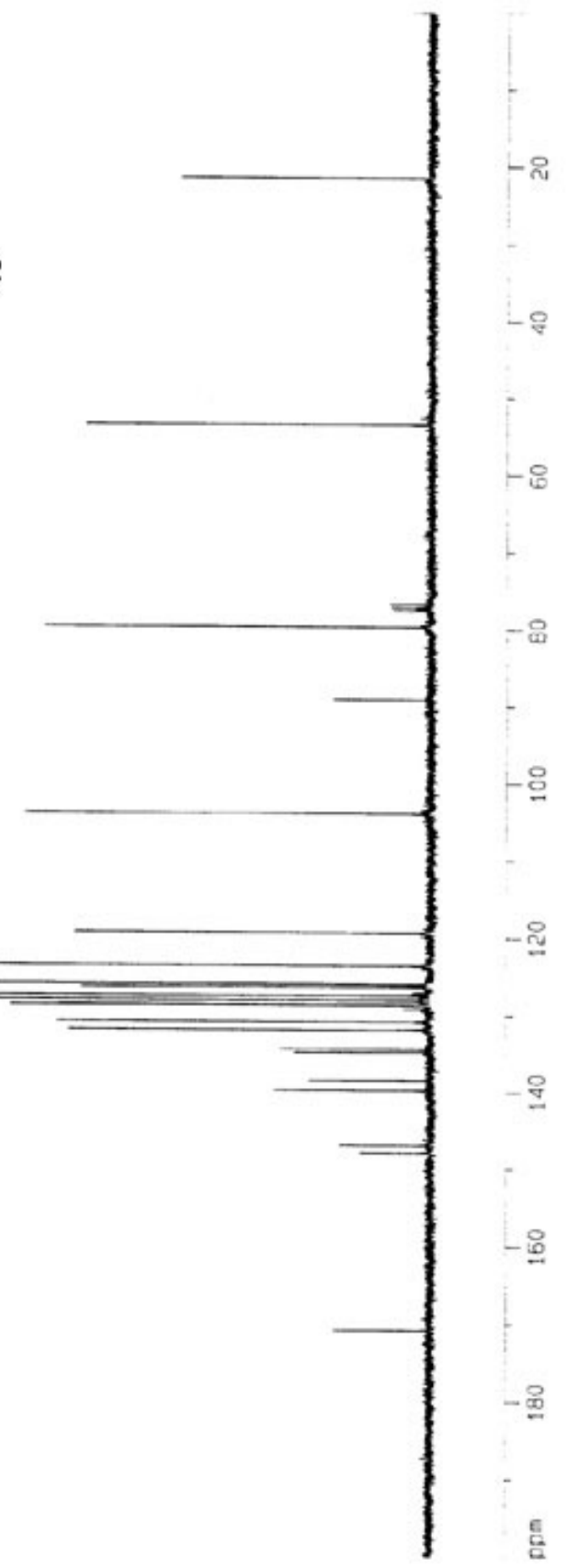



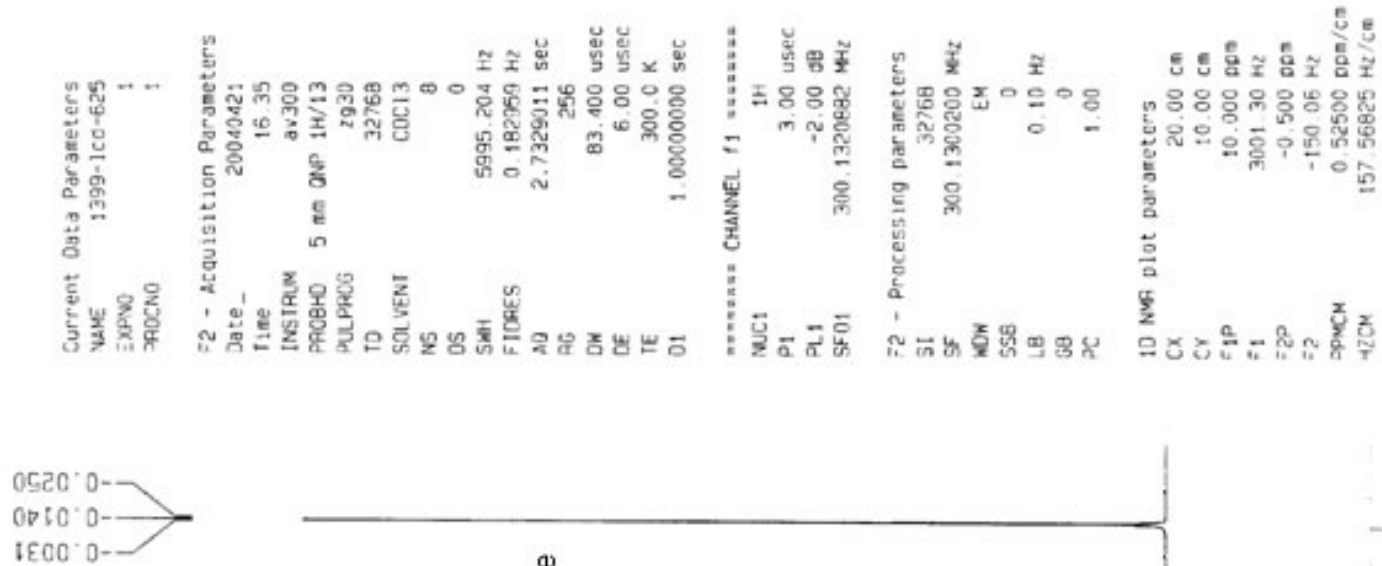

269E'

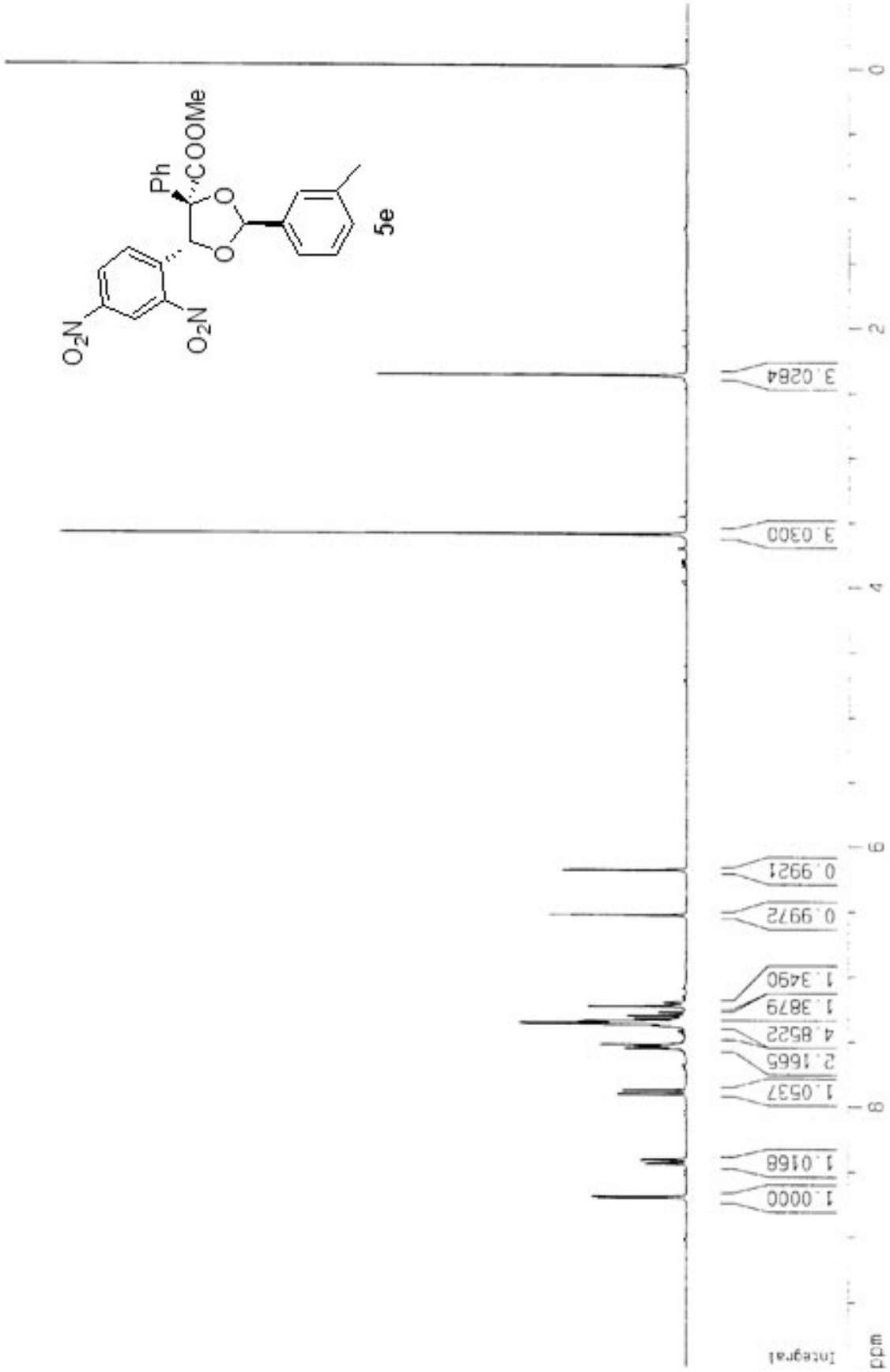

885 ' $E$

$0965^{\circ} \mathrm{E}$

SLOL'E

SEEL'E

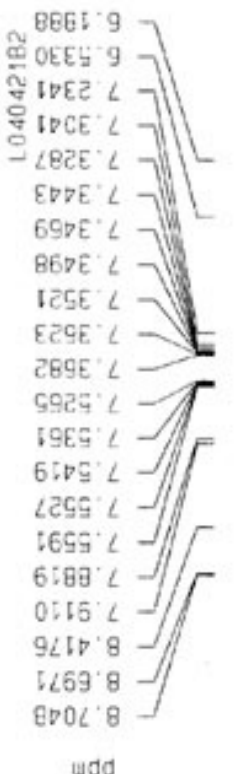




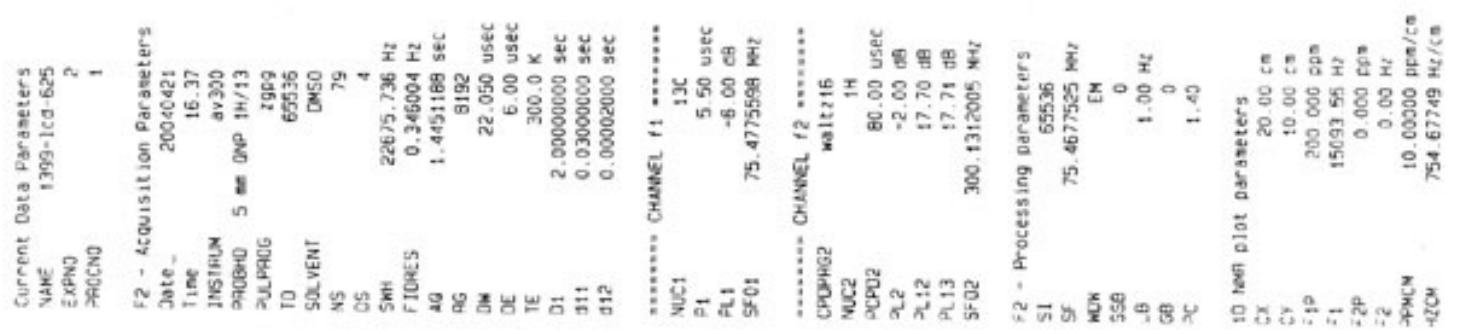

Oto 12

6ट8'29

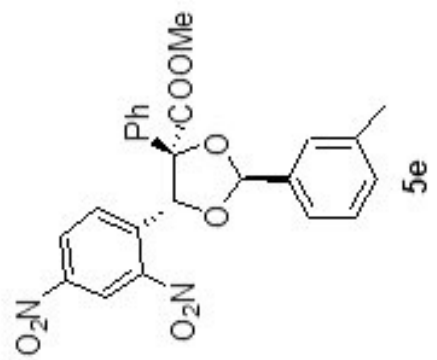

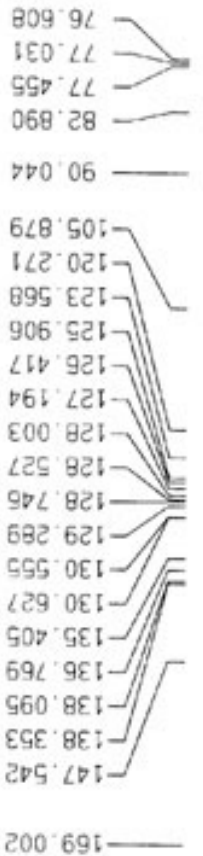

wdd

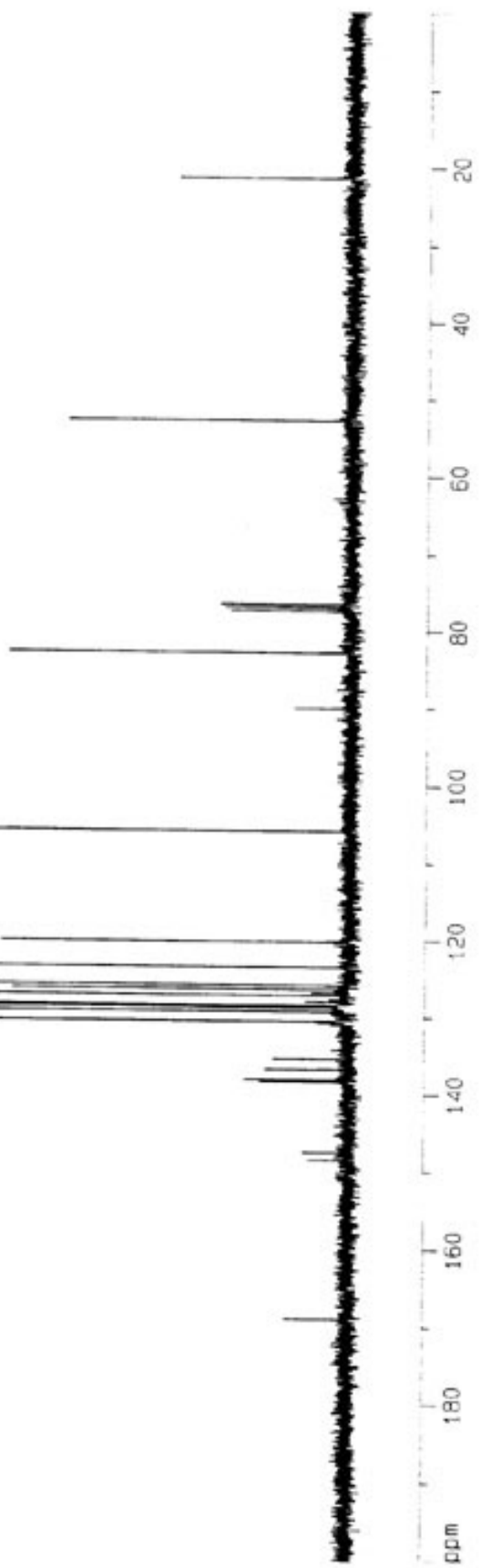




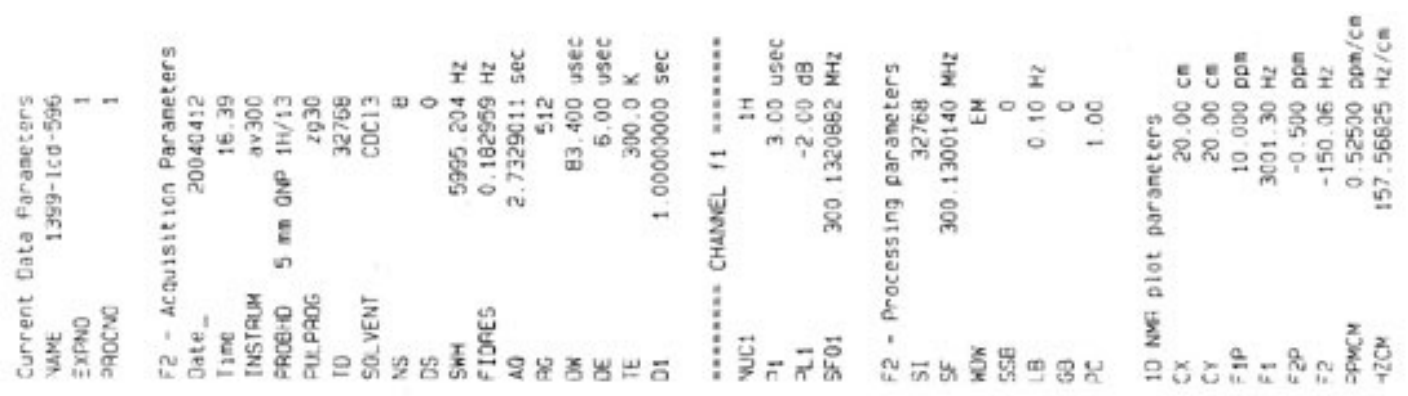

$E 210.0$

$E 100^{\circ} 0$

$\nabla 6000$

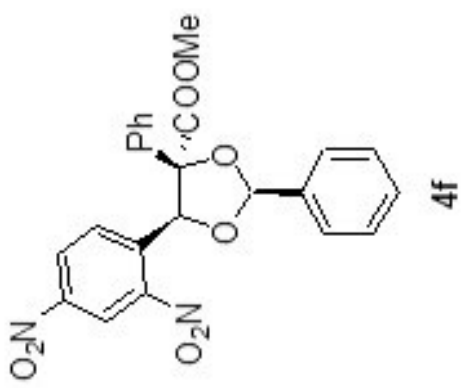

DटGब $\varepsilon->$
$06 \angle 5^{\circ} \varepsilon->$

$=\sqrt{69866^{2}}-7$
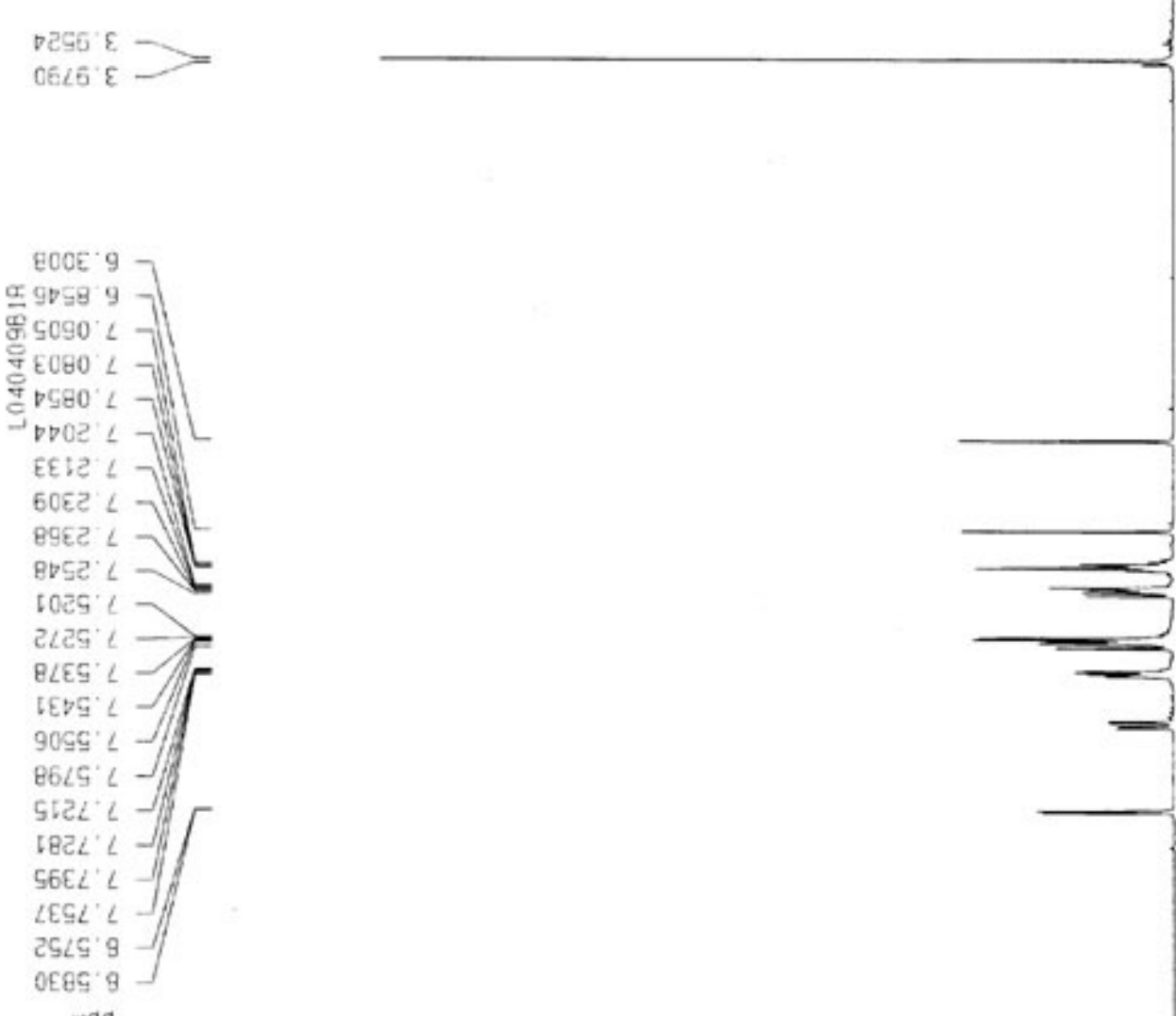

แda

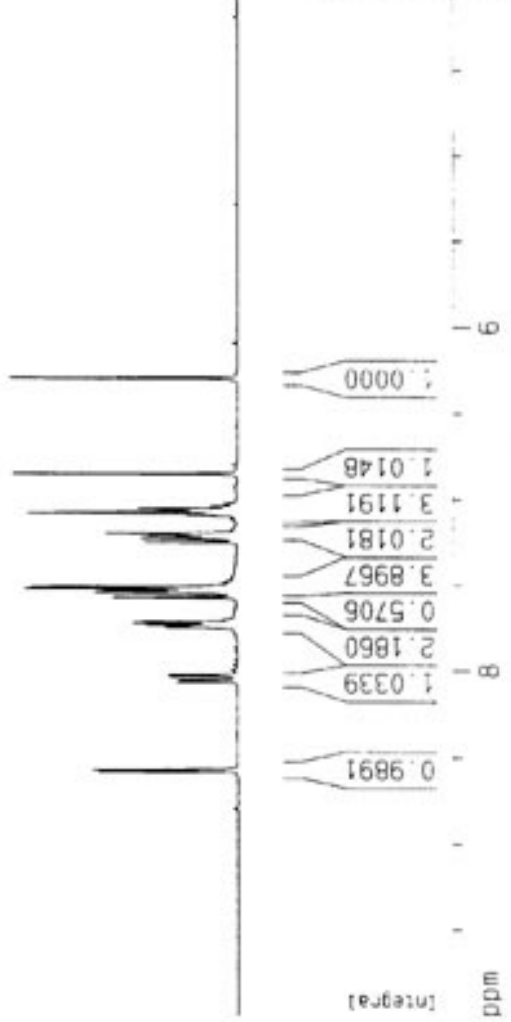




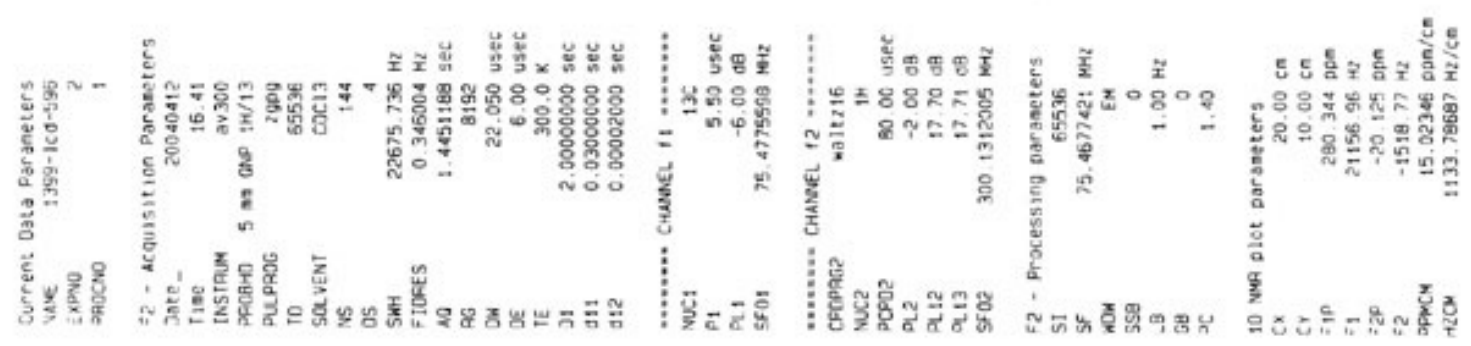

8150
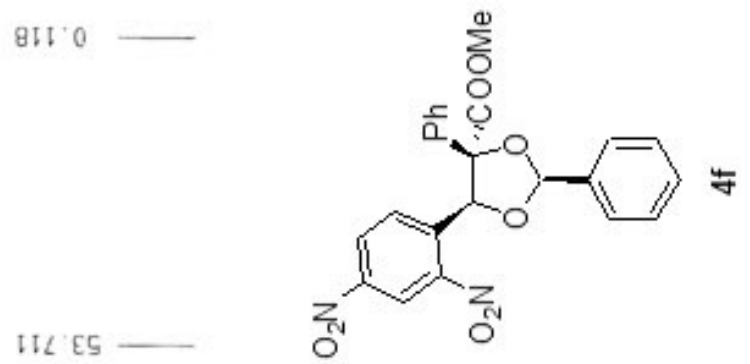

SEL' $9 L$

$\angle S V^{\prime} \angle L$

$285^{\circ} \angle L$

6हB $6 L$

OLE $68-$

SL6 EOD

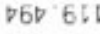

090.925

$18 B-9 \overline{1}$

¿दB 9 व

$\angle \angle L, 8 E 5$

66L 8टा

ОЕ6 घटा-

DUE CEI-

200 टEL

SटD $\nabla E !$

986 DE!

268 $6 \mathrm{E}-1$

$2 \angle 0<01-3$

5डा 801$]$

$9060<1-$

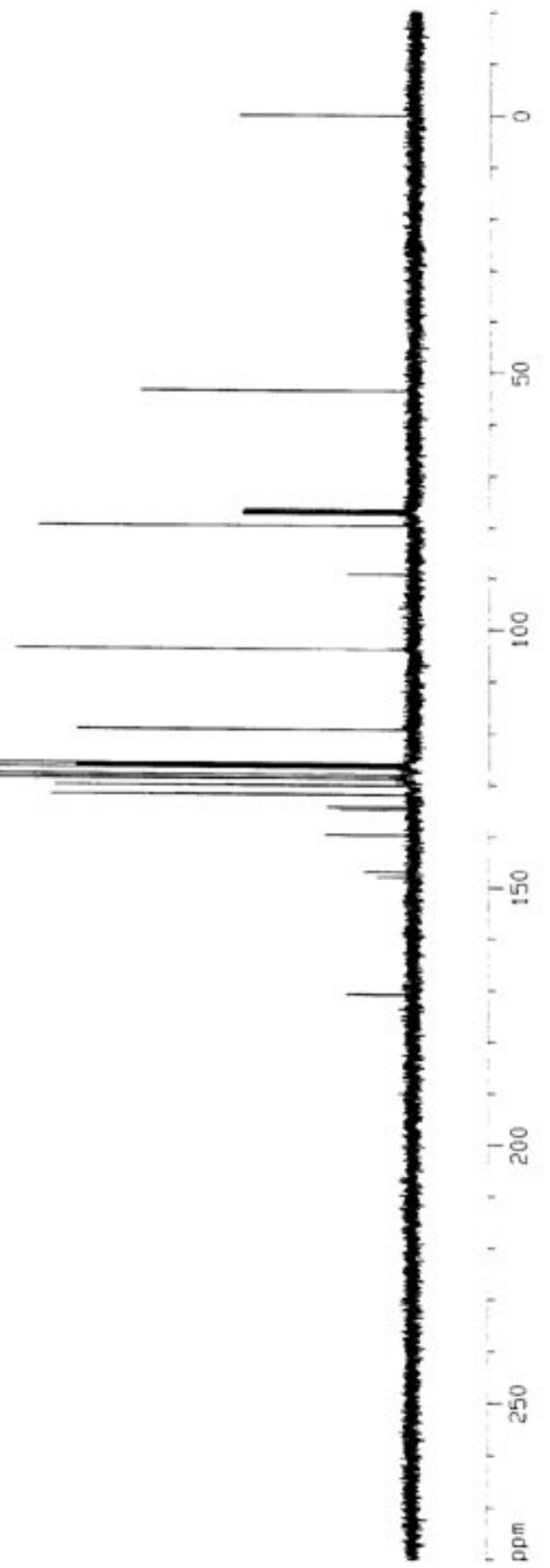



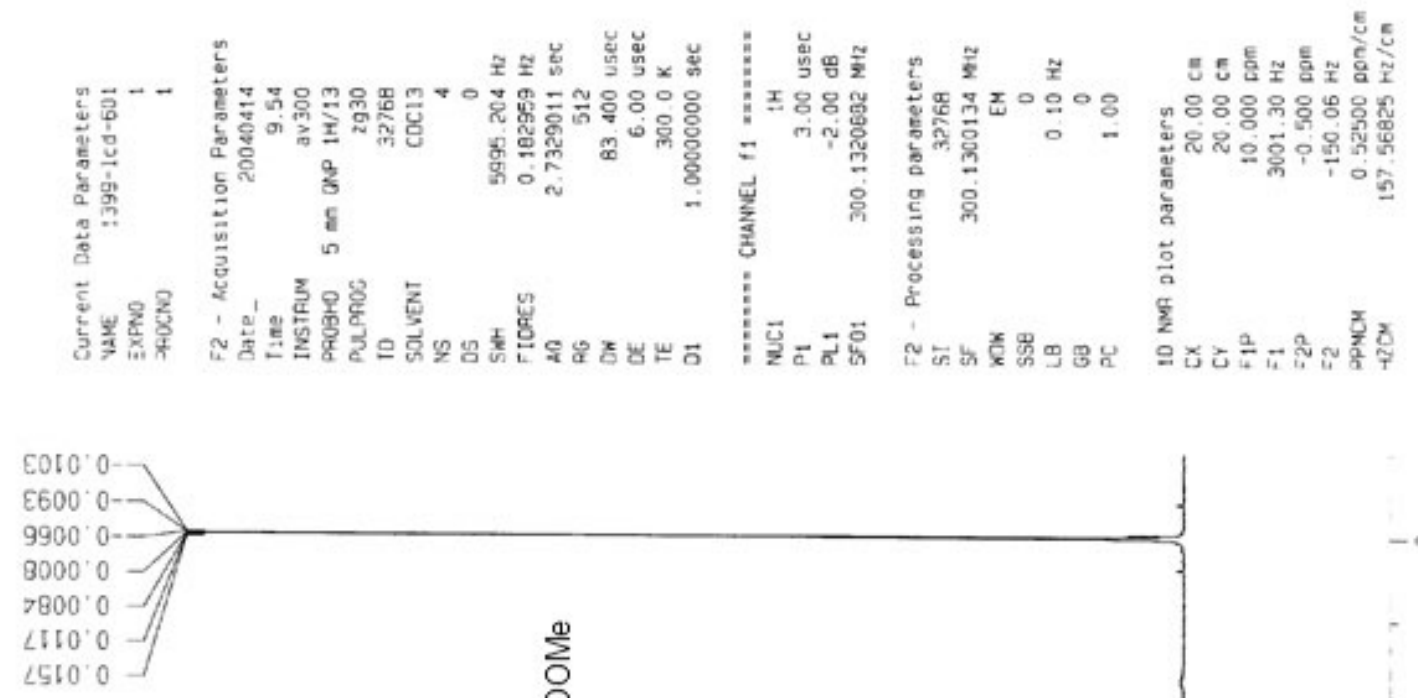

66/9':

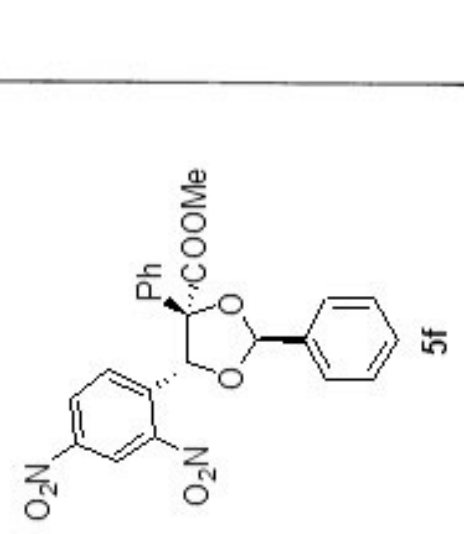

$6619 \varepsilon$

CLCL ह -

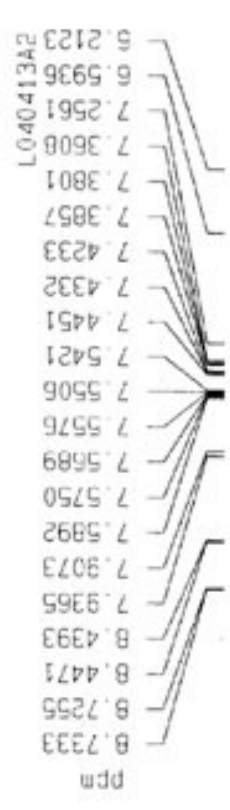

\section{गटा ह}

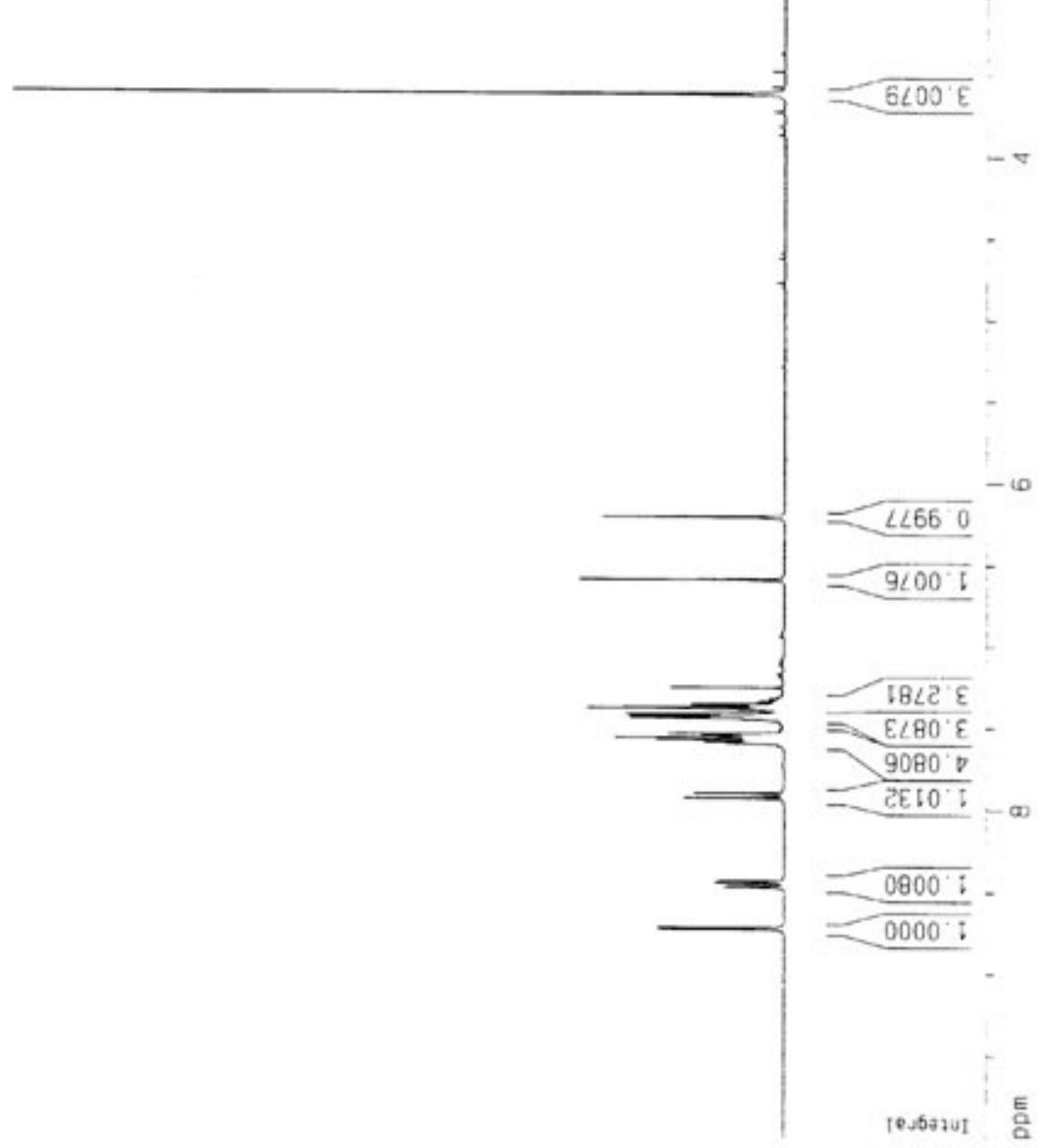




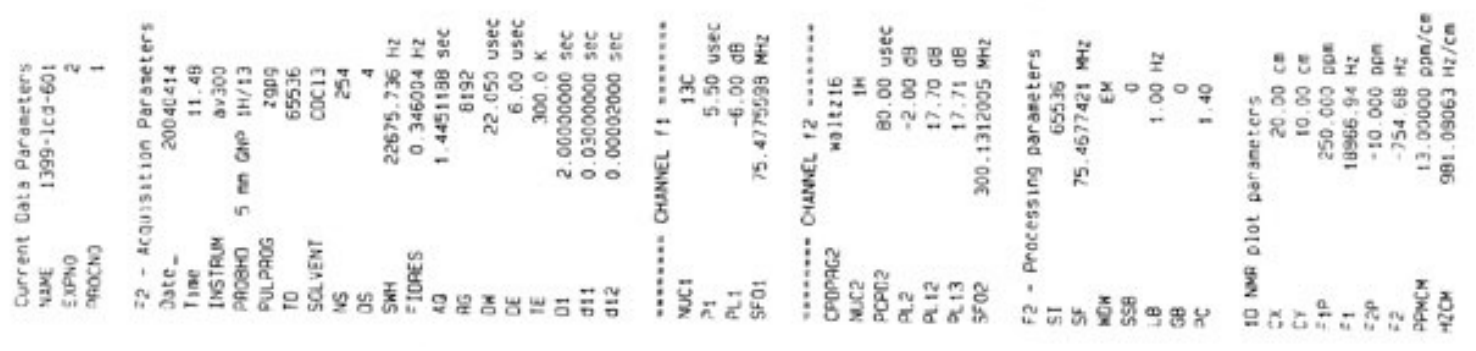

921 0

Sto Eg

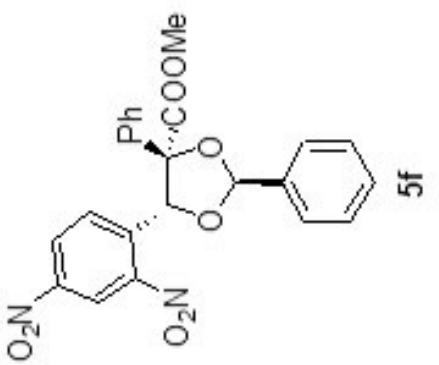

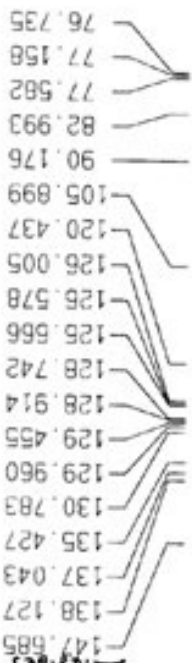

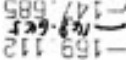

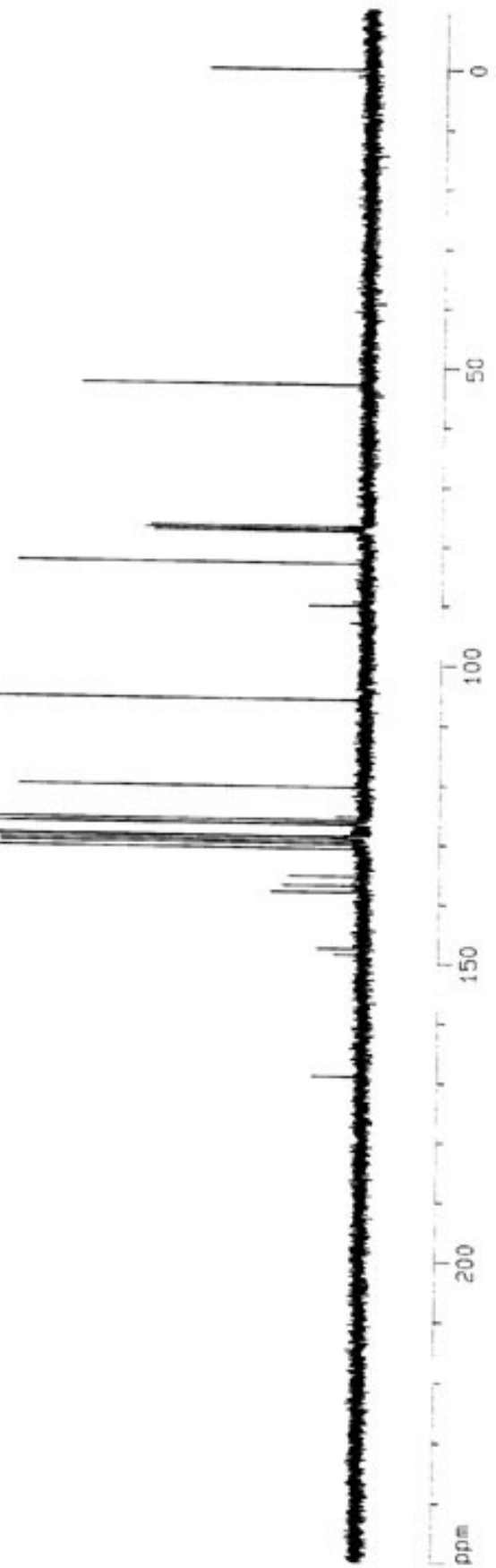



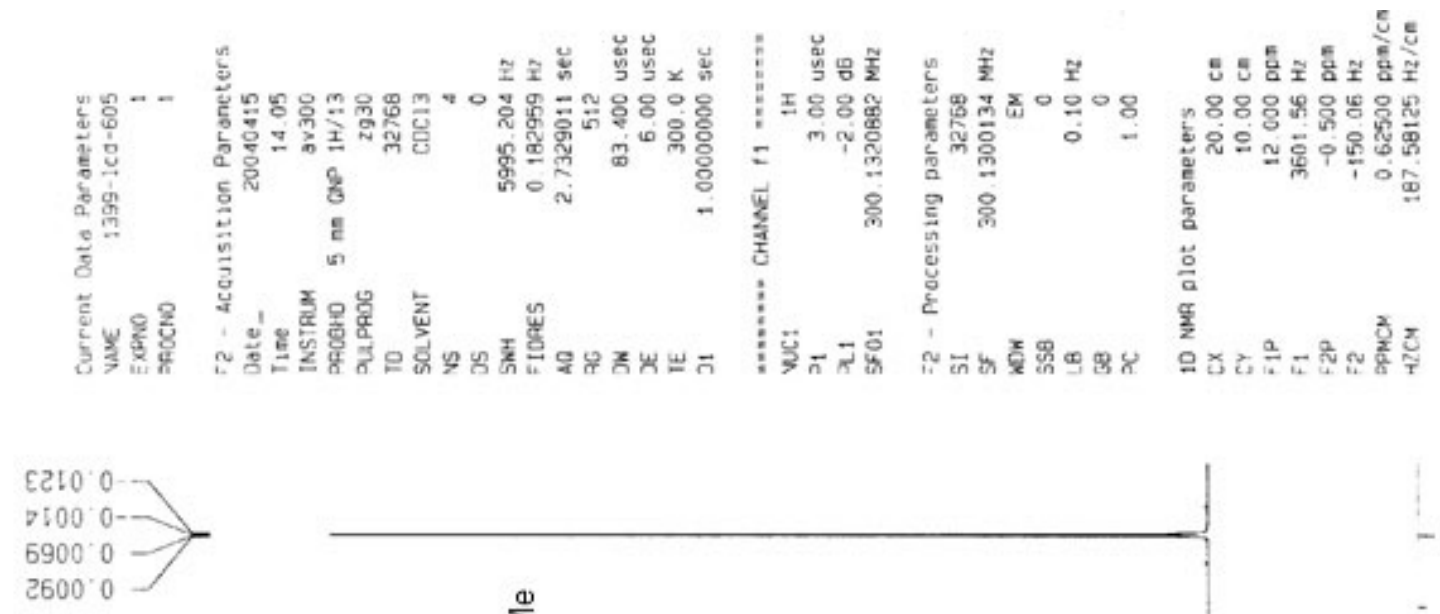

2289
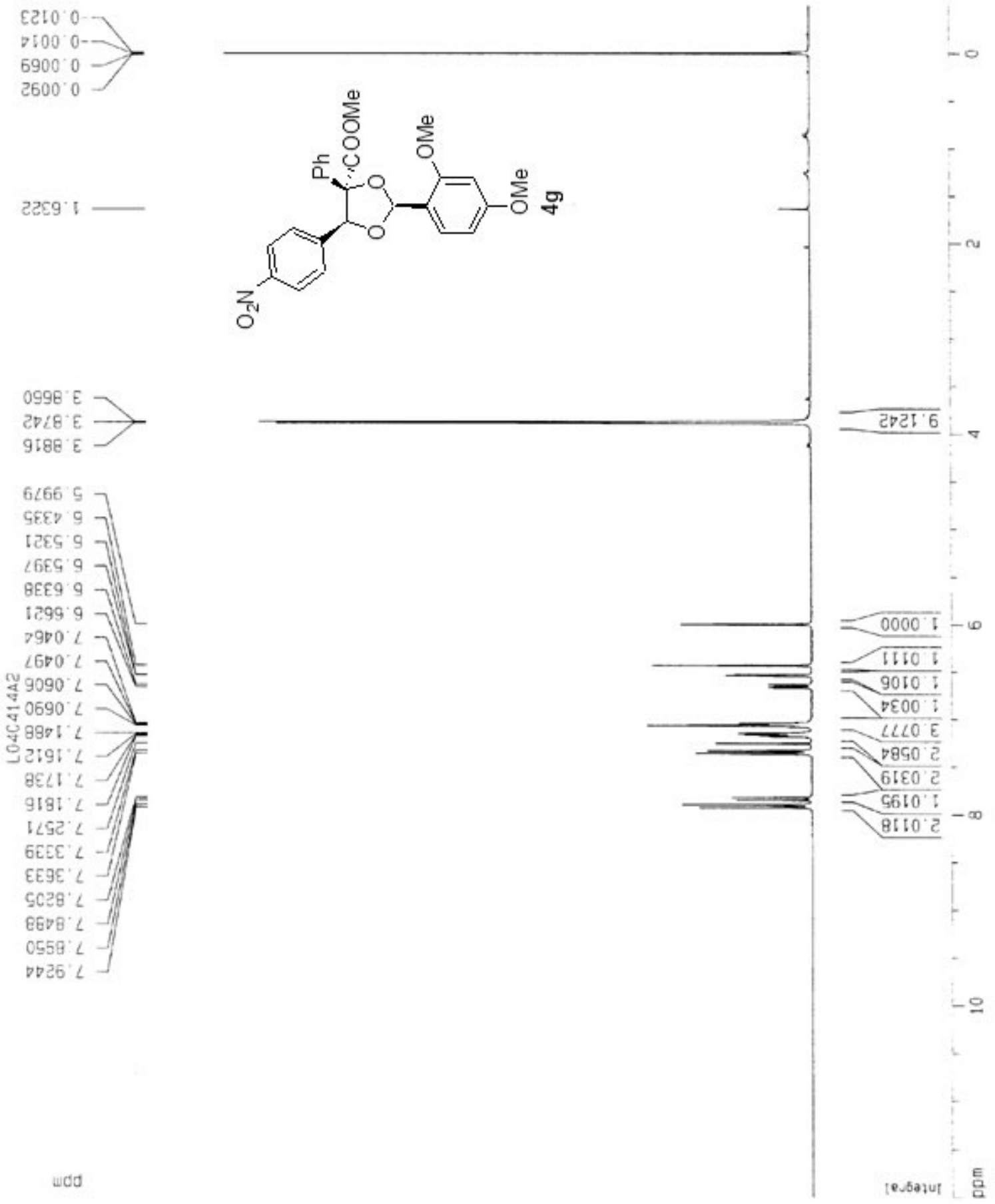


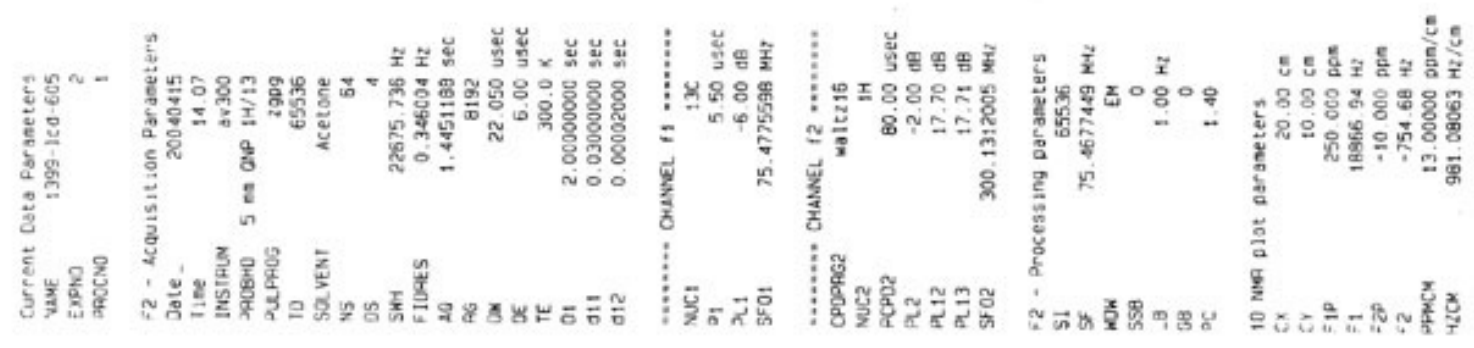

$680^{\circ} 0$
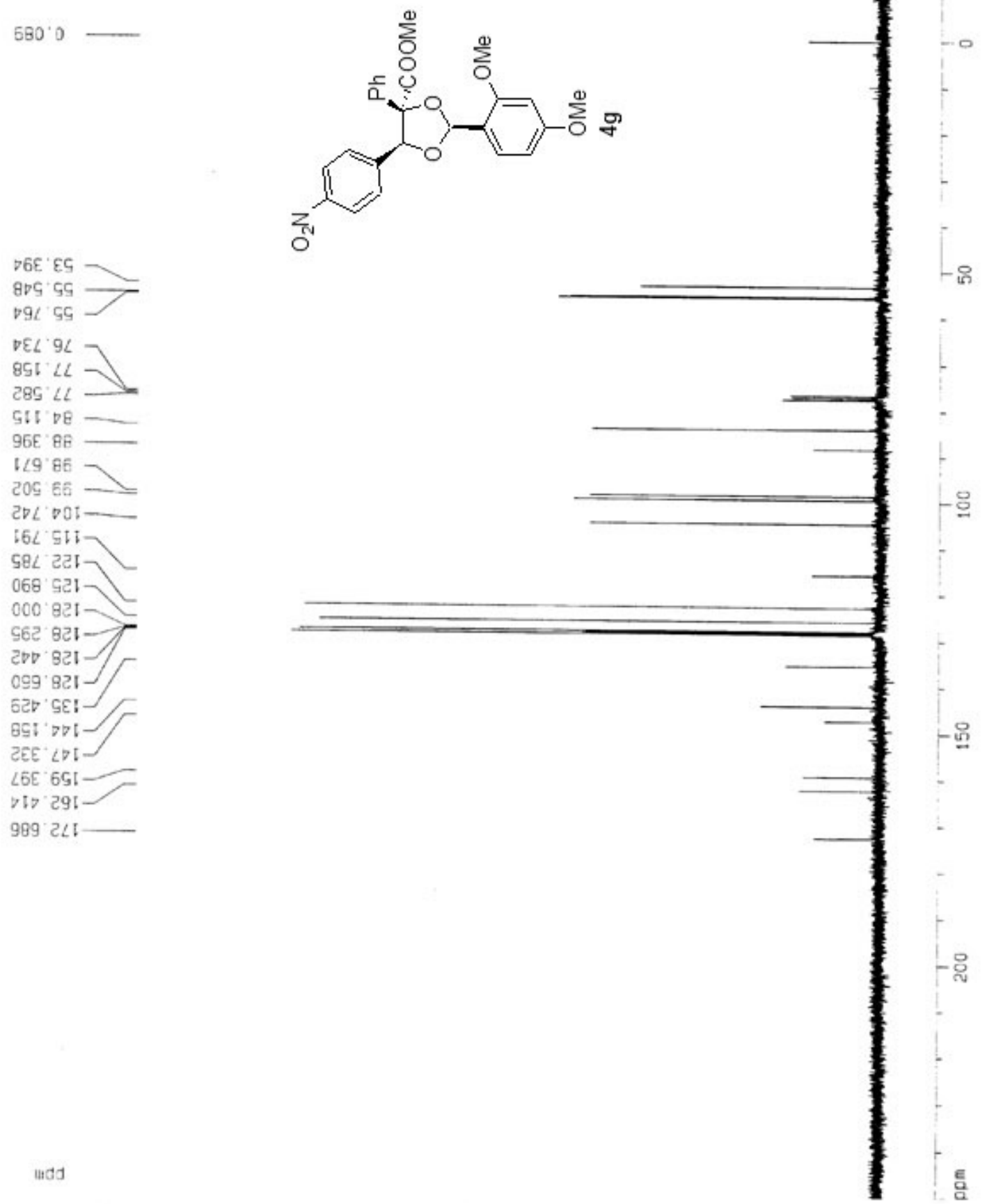

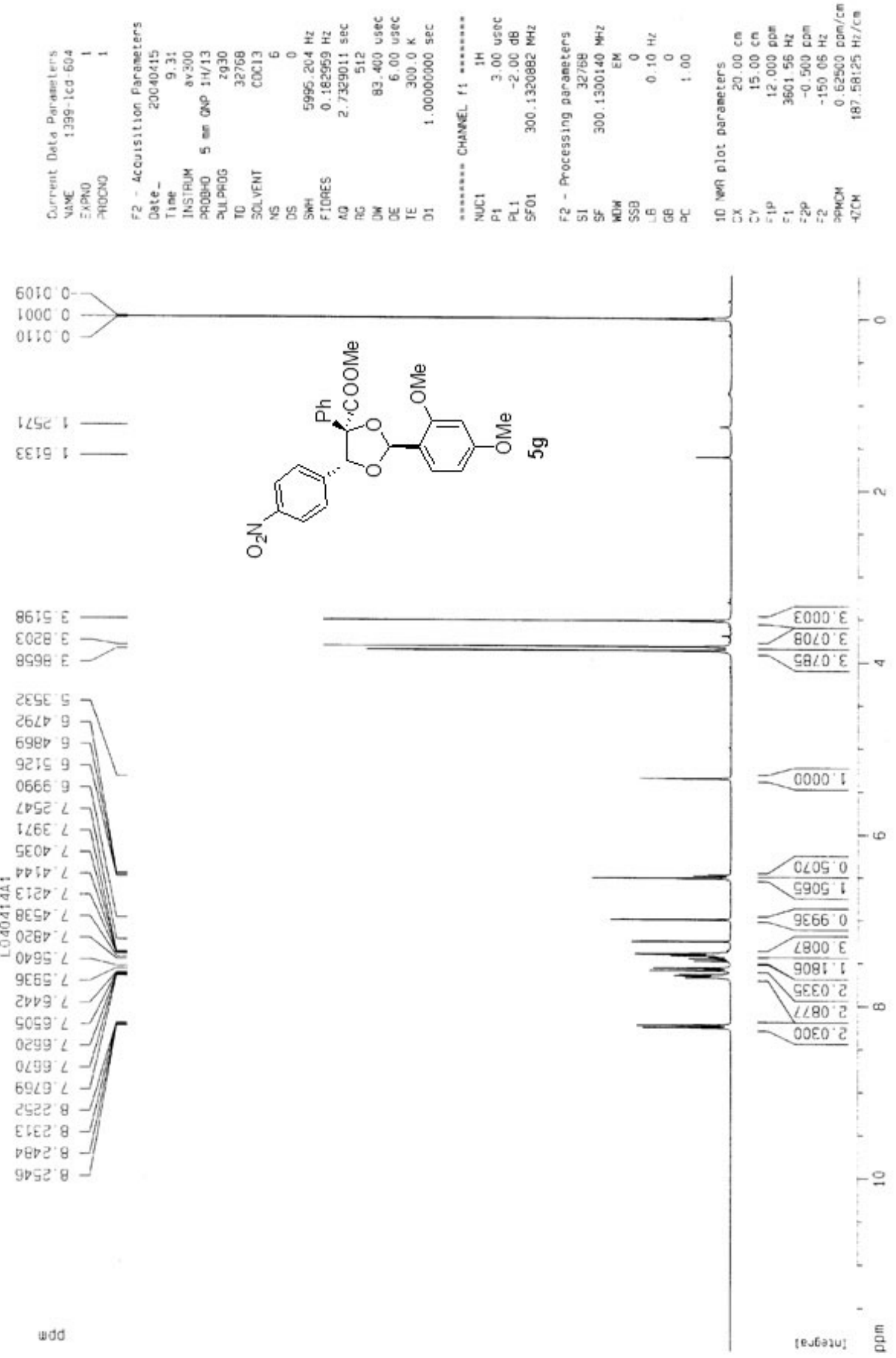

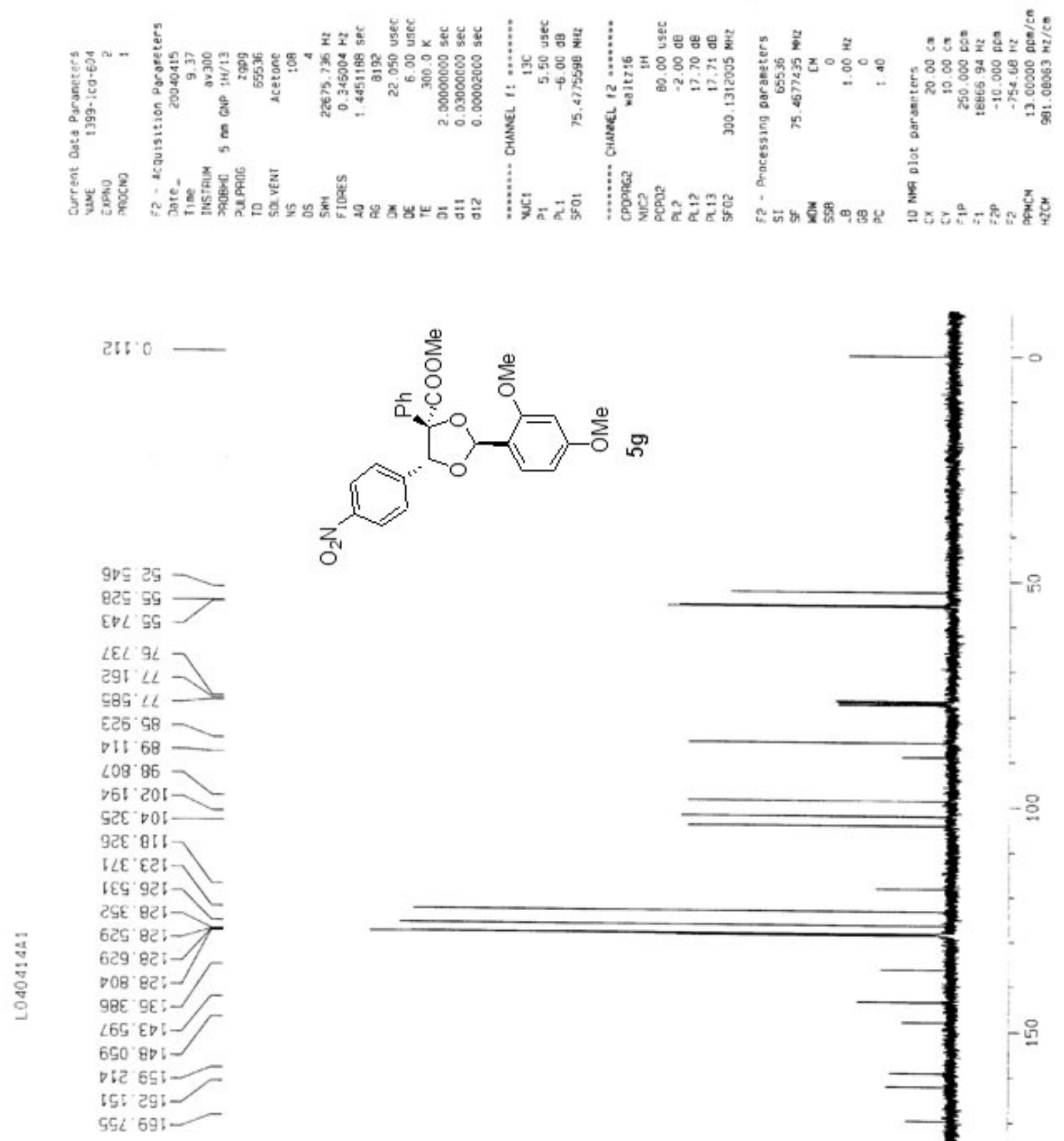

$9052 S$
$829 \mathrm{GS}$
EDL $\mathrm{CS}$

$\angle E L G L$

9टE' 8 เ

$625 \cdot 82$ :

629 ' 82 :

650 80 :

7 iट 65 t

G5: $691-$

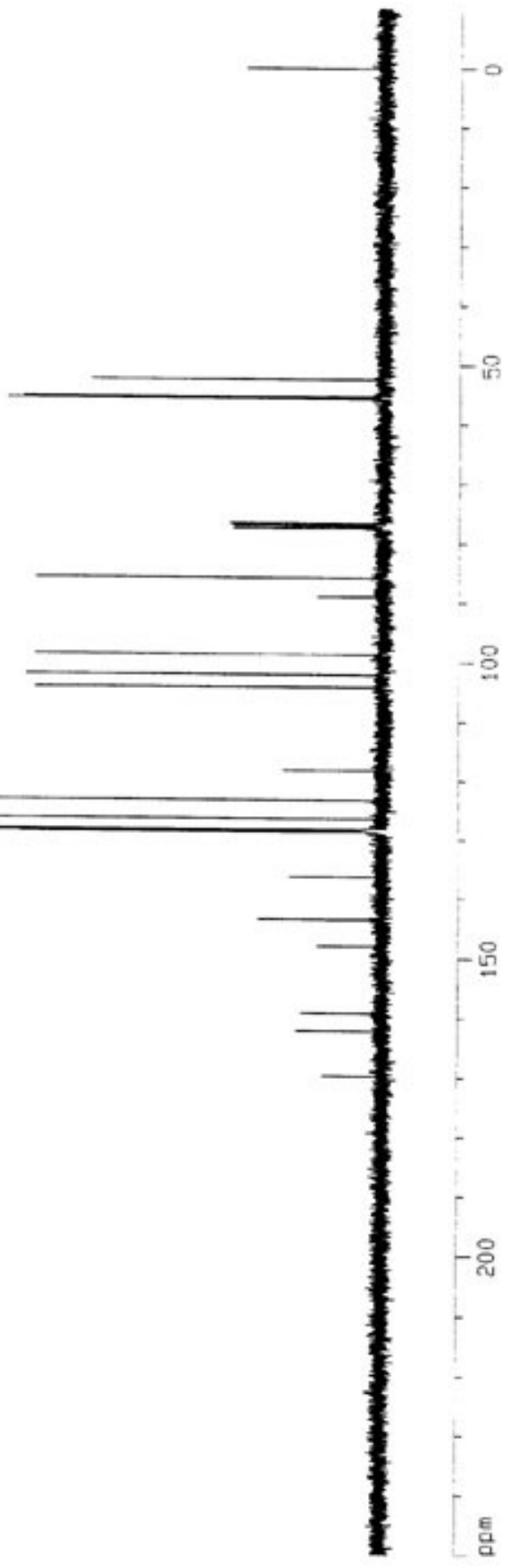



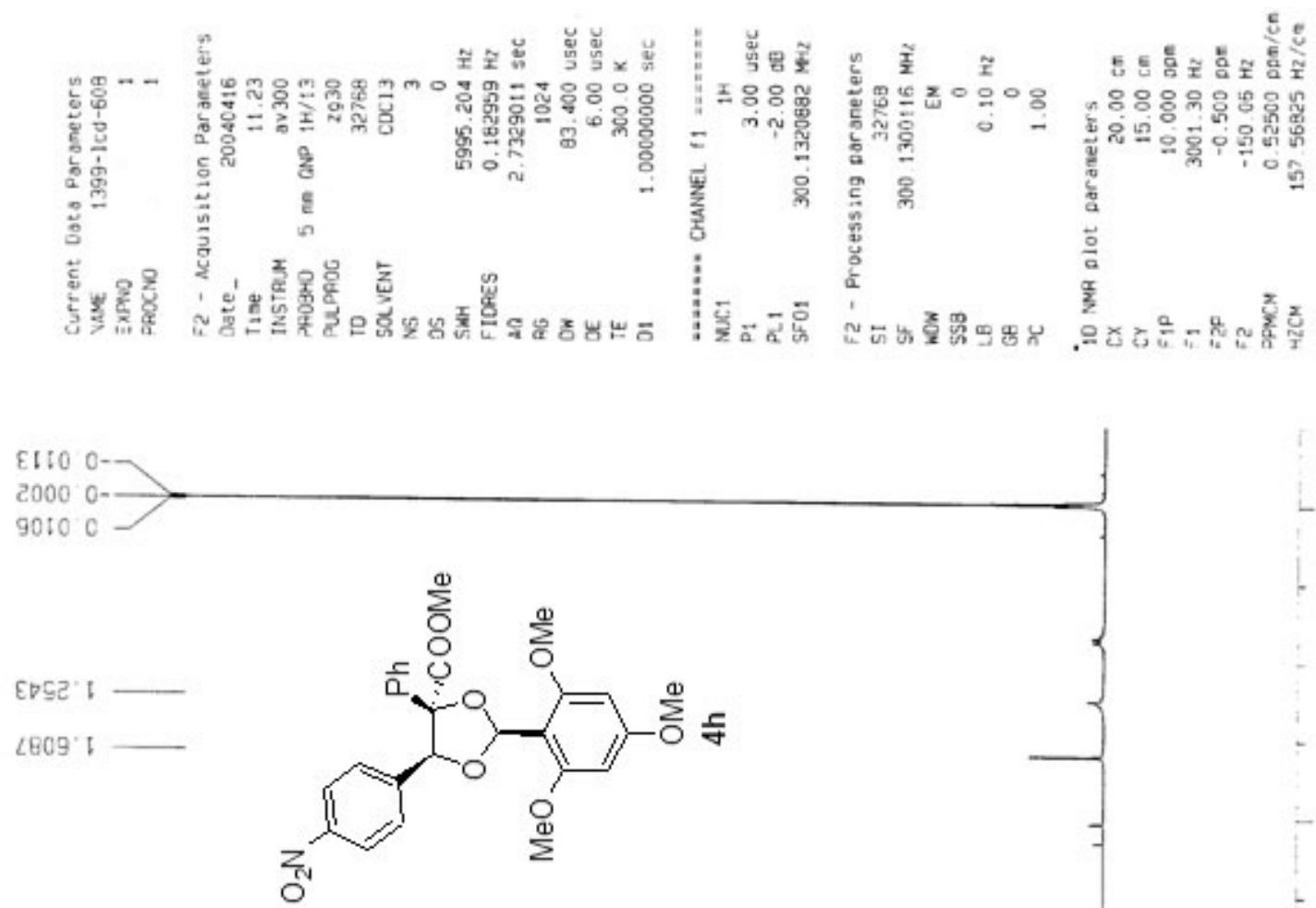

हटऽ8 E

SU $\angle \theta^{\circ} E$

I 68 '
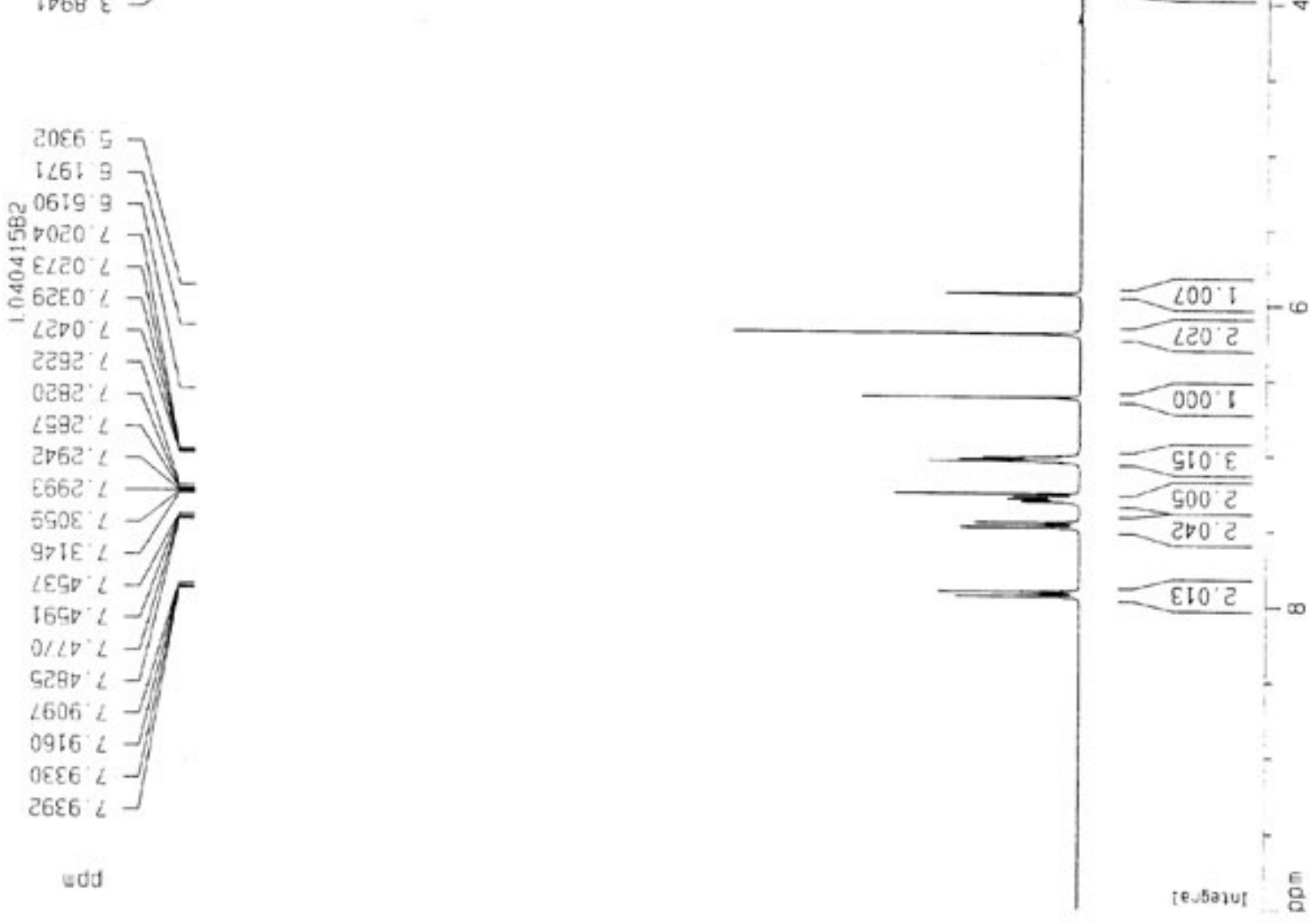


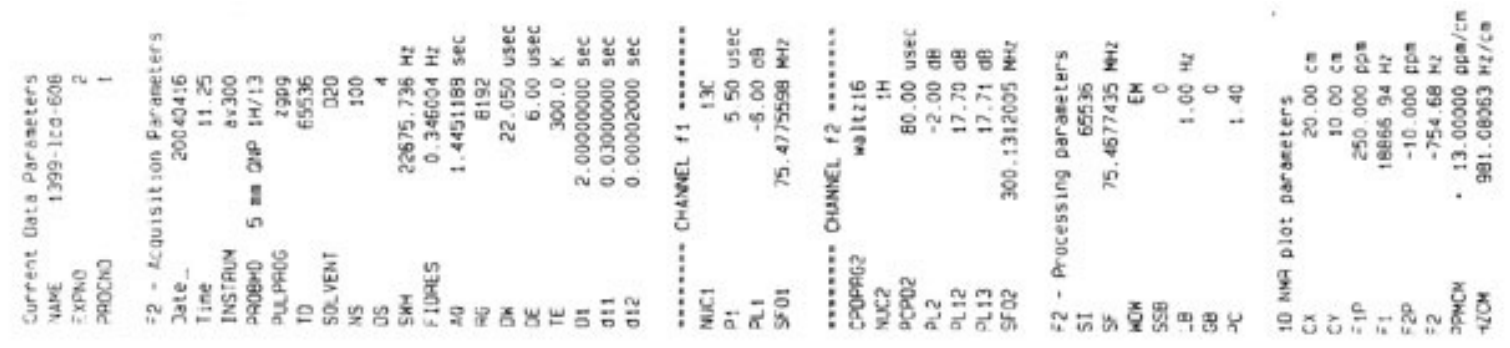

6600

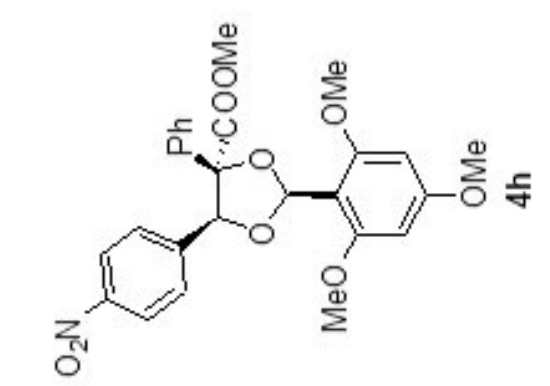

$\angle 2 E$ ES
$6 \angle 0$ SG
OEg SS

टह! '9!

sदा' $L L$

$6 \angle 5^{\circ} \angle L$

टट0 $\nabla 8$

$\angle 26 ' \angle 8$

9E6 06

EटL' 86

SB2' $201-$

$10 L .521$

112.921

$629 \circ 221$

$2 \angle 6 \div 2$

128.825

$1 \angle 8 \mathrm{SEI}$

$170 \mathrm{SDI}$

$\checkmark 0 E^{\circ}$ เ 9 -

EEI EgI-

$88 S^{\circ} \varepsilon \angle L$
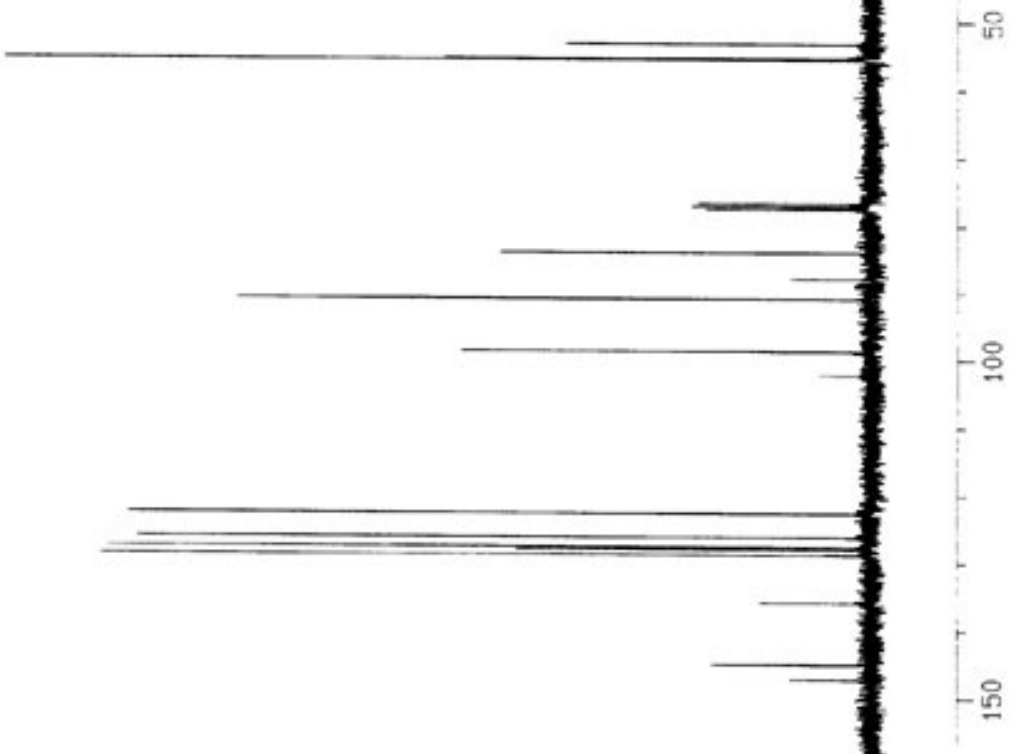

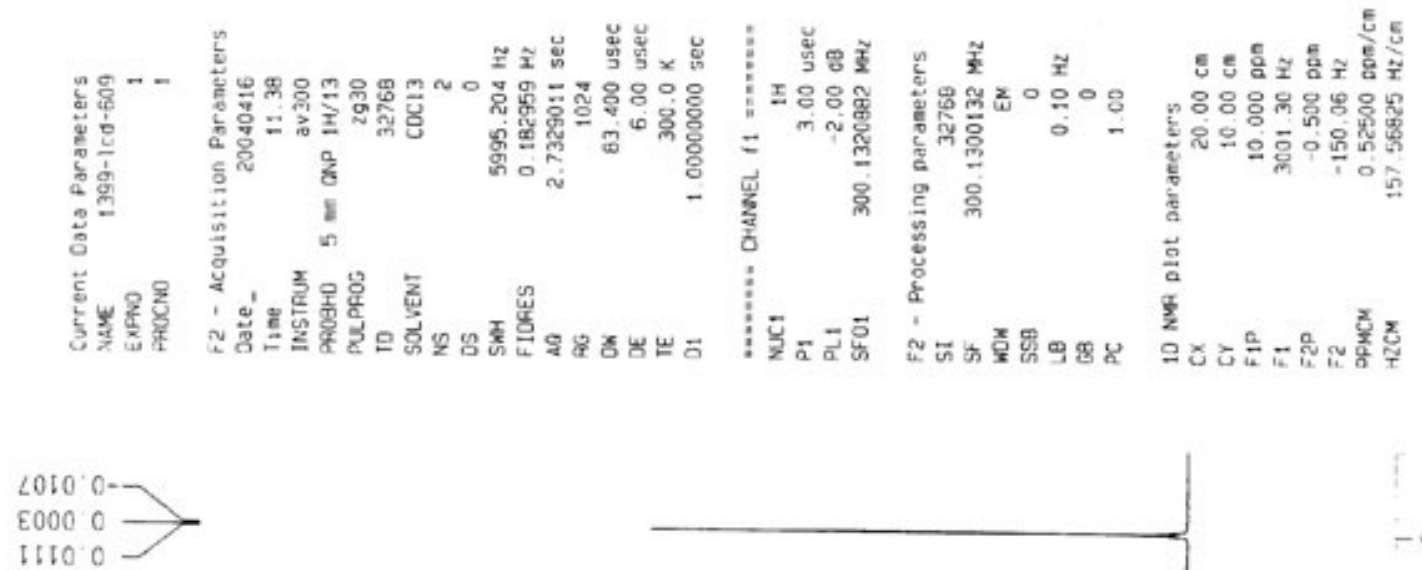

D029:

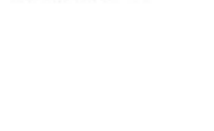

7 SES $\varepsilon$

t59 $\varepsilon$

EEट马 $\varepsilon$
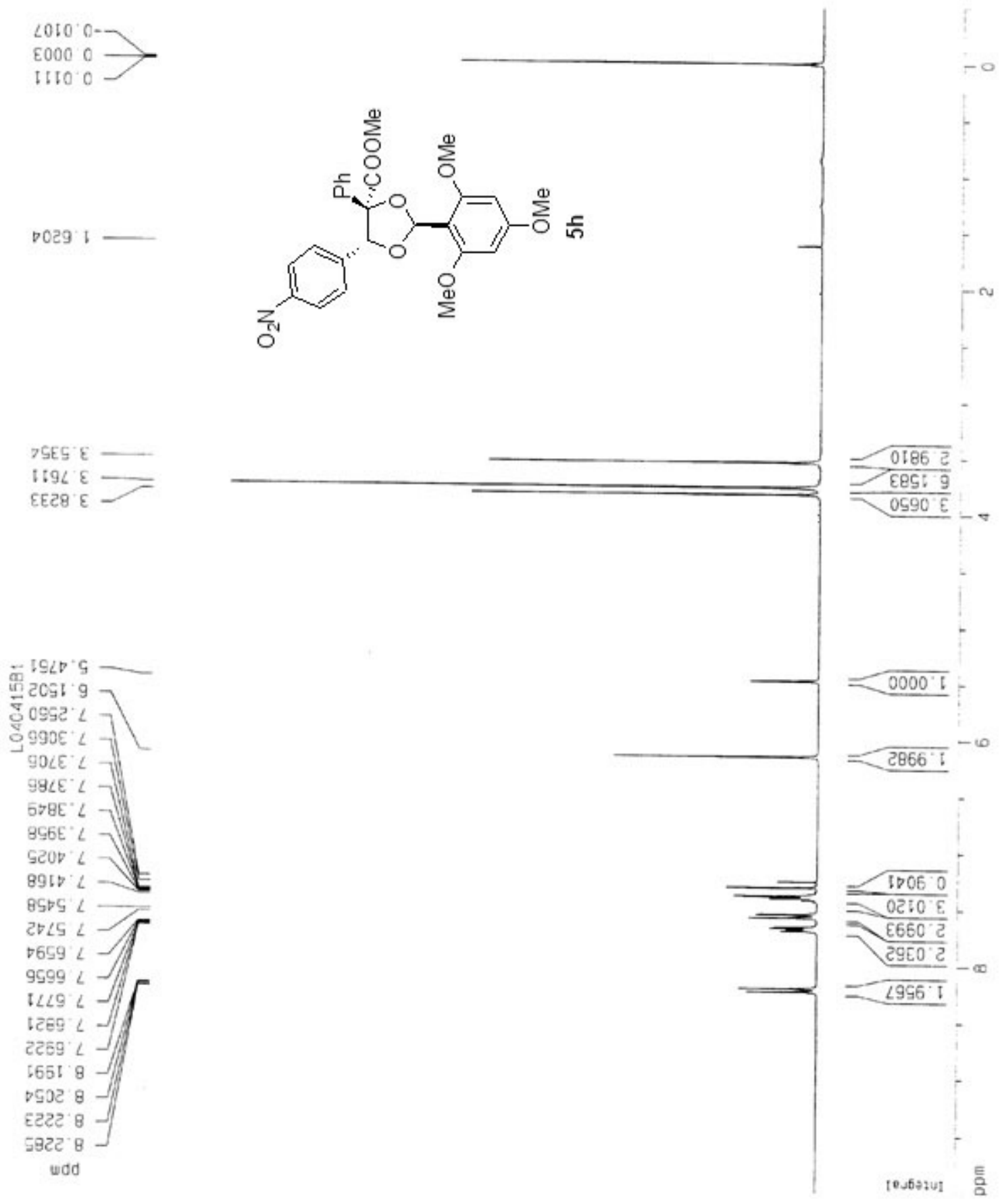
S46

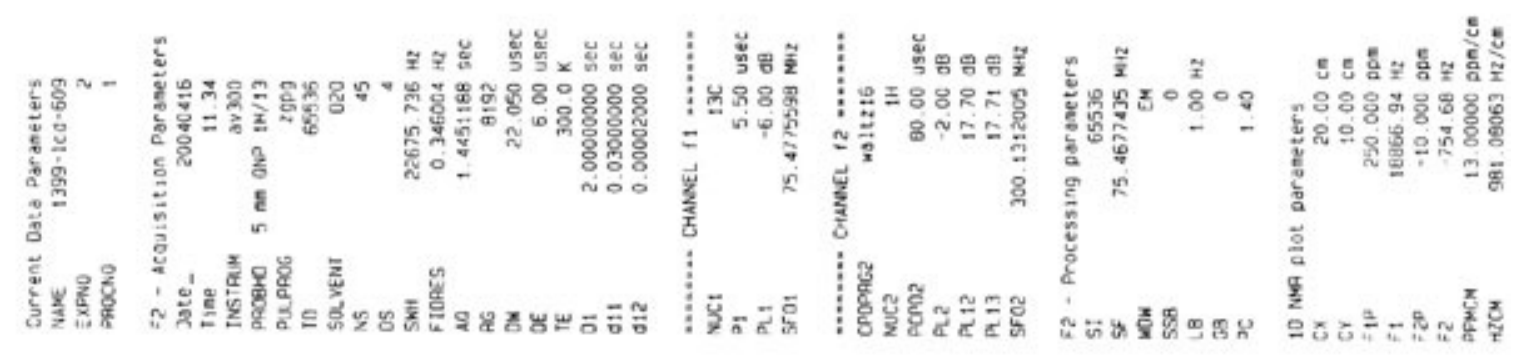

$600^{\circ} 0$
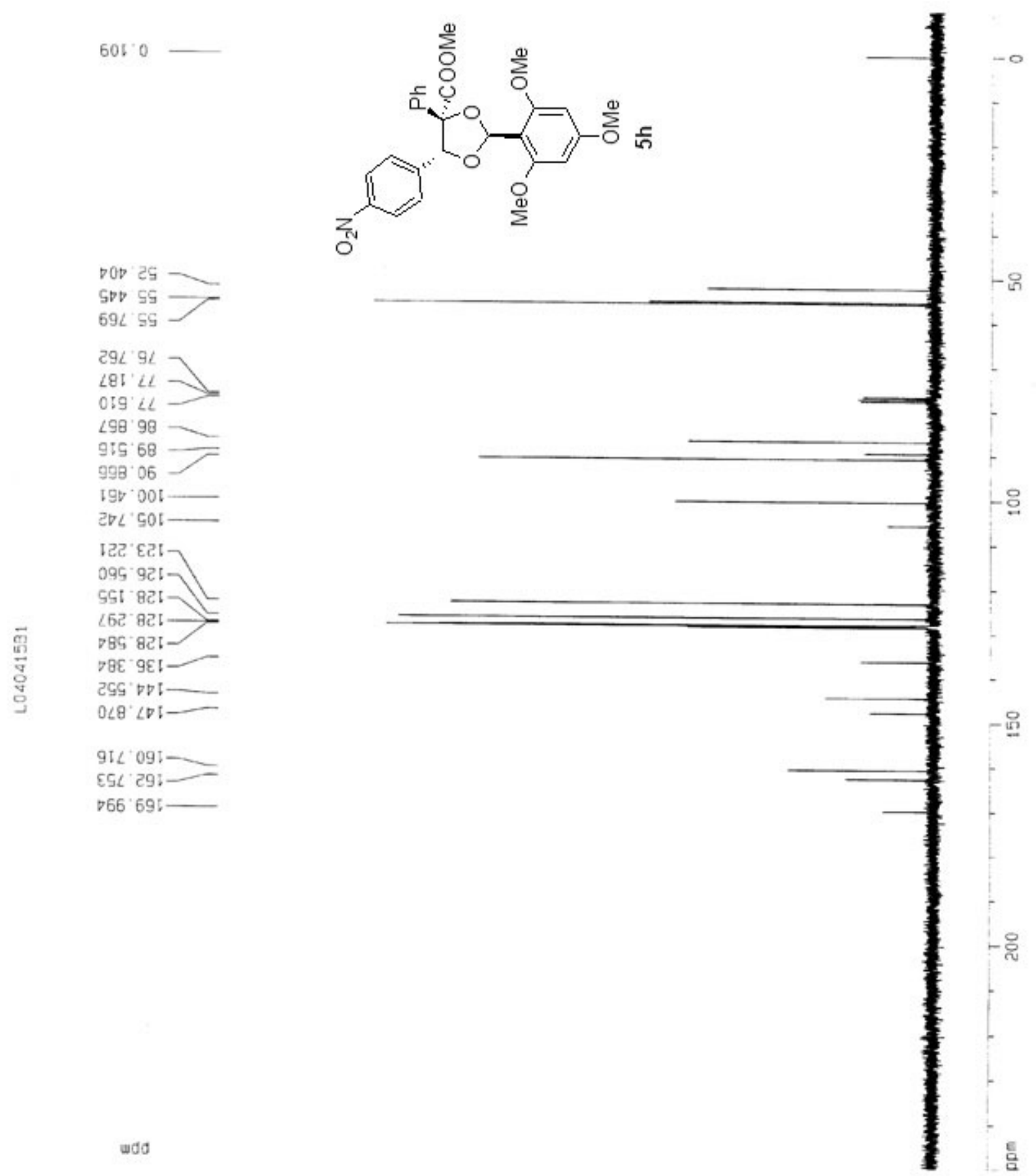


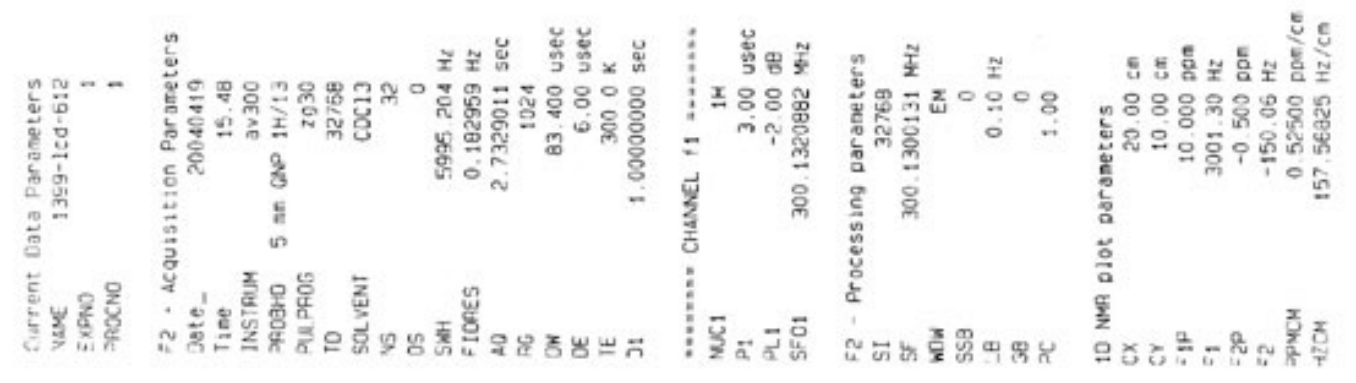

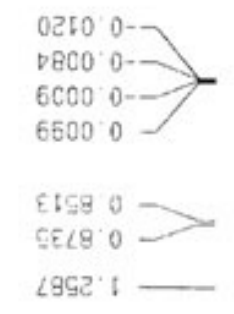

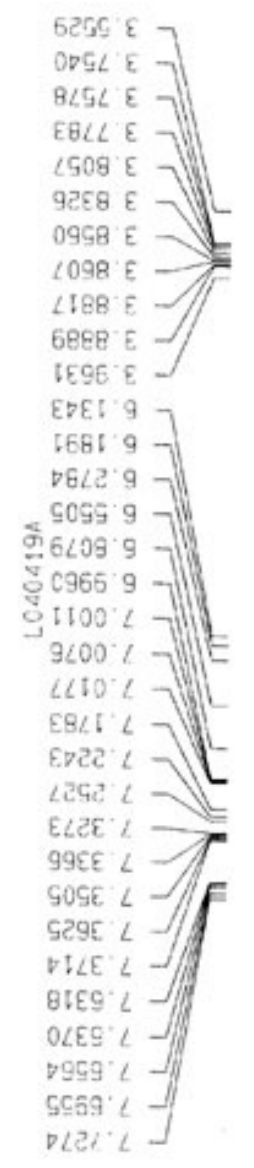

udd

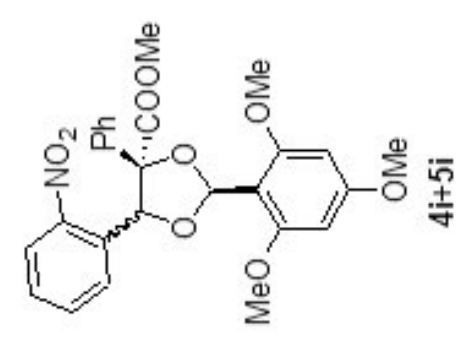

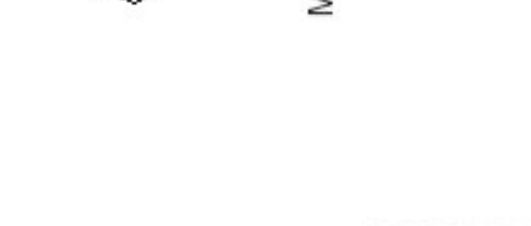




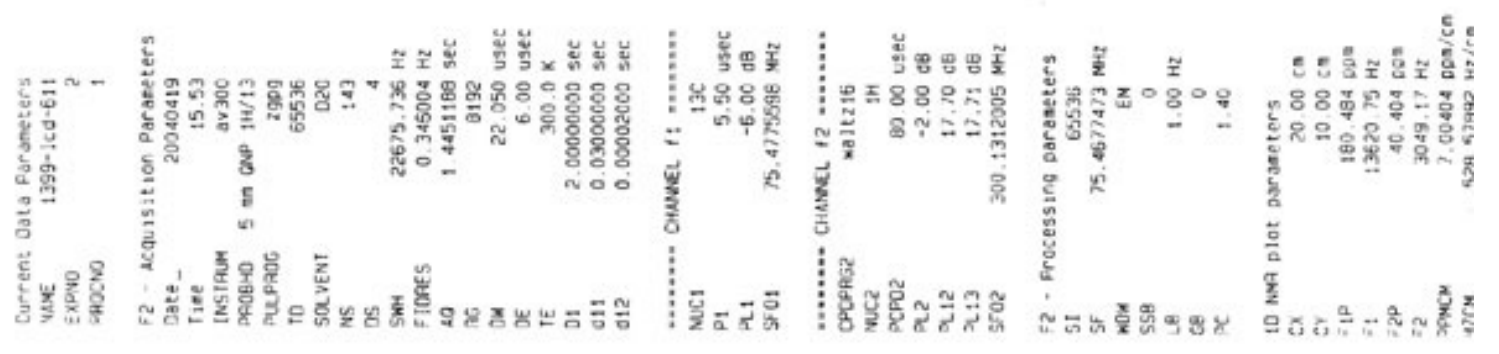

S62' 29

9टा ह5

ट9ع 55

VIV SS

टES SS -

$\angle 9955$

टEL $9 L$

$95 \mathrm{~T}^{\circ} \angle L$

BSE $\angle L$

$185 \angle L$

$\angle D O^{\circ} O B-1$

$02 \angle \mathrm{CE}$

$269^{\circ} \angle 9$

20958

SGL 05

E68 05

$97 \subseteq$ 8ธิ

BदE 001

DQE टOT

OटB 901

coe.25I

E:L

म05 $951-$

गटL' $921-$

$\begin{array}{ll}E 60^{\circ}<2 t \\ E 15 & <1]\end{array}$

ort $82 \mathrm{~L}-1$

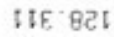

ह6ด 825

$610^{\circ} 62 t-$

$\nabla 99^{\circ} 62 !-$

gEg OE: -

D 60 '

จ8เ टहा-

เ9ट' टह!-

टટ० 'દE: -

૬बเ $9 \varepsilon:-$

จटे $9 \varepsilon:-$

265 $80:-$

sम2 $60:-$

$\angle 69$ 09:-

म92.tet

IEg दI-

GटO Egt-

$900 \mathrm{CLI}$

เด: $2 \angle 1-$

udd

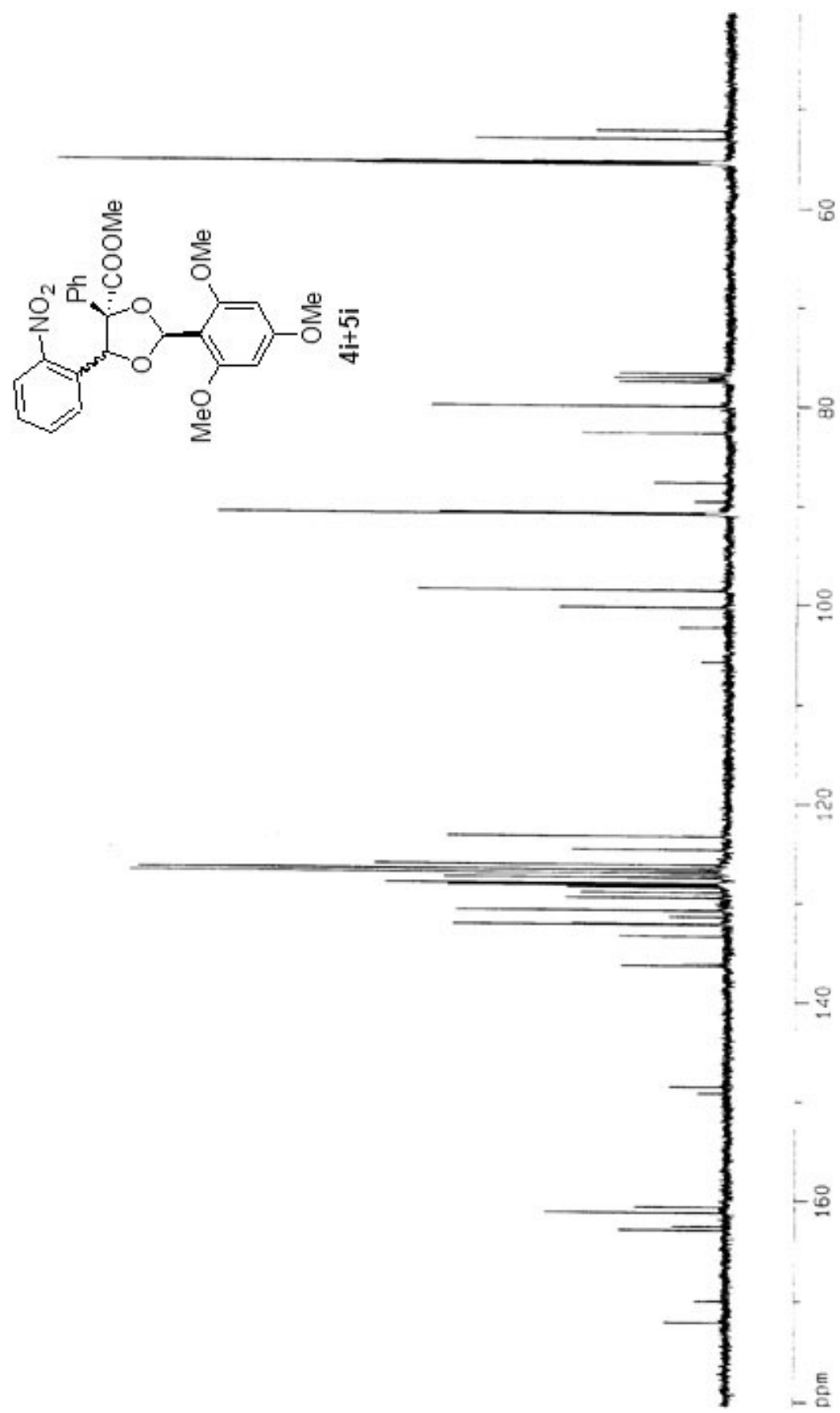




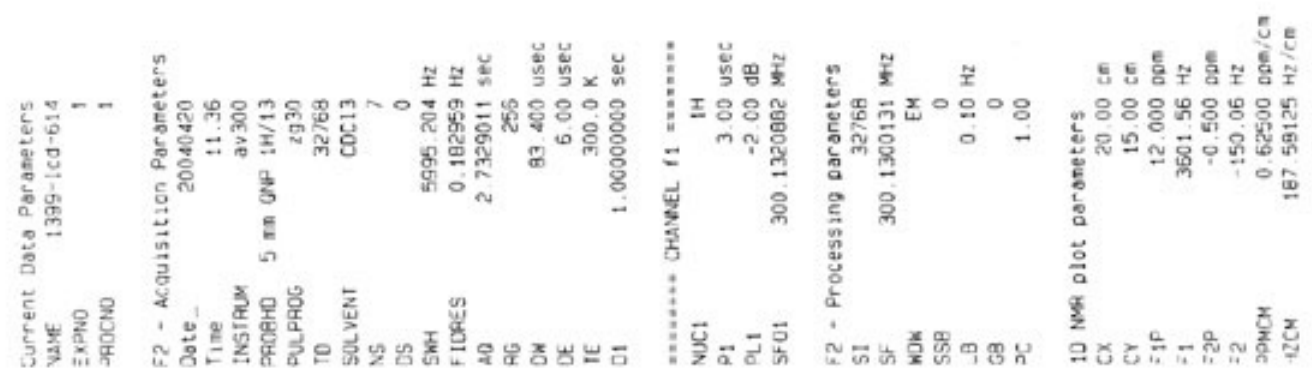

5250.3

$11000-$

$\angle 6000$

2219
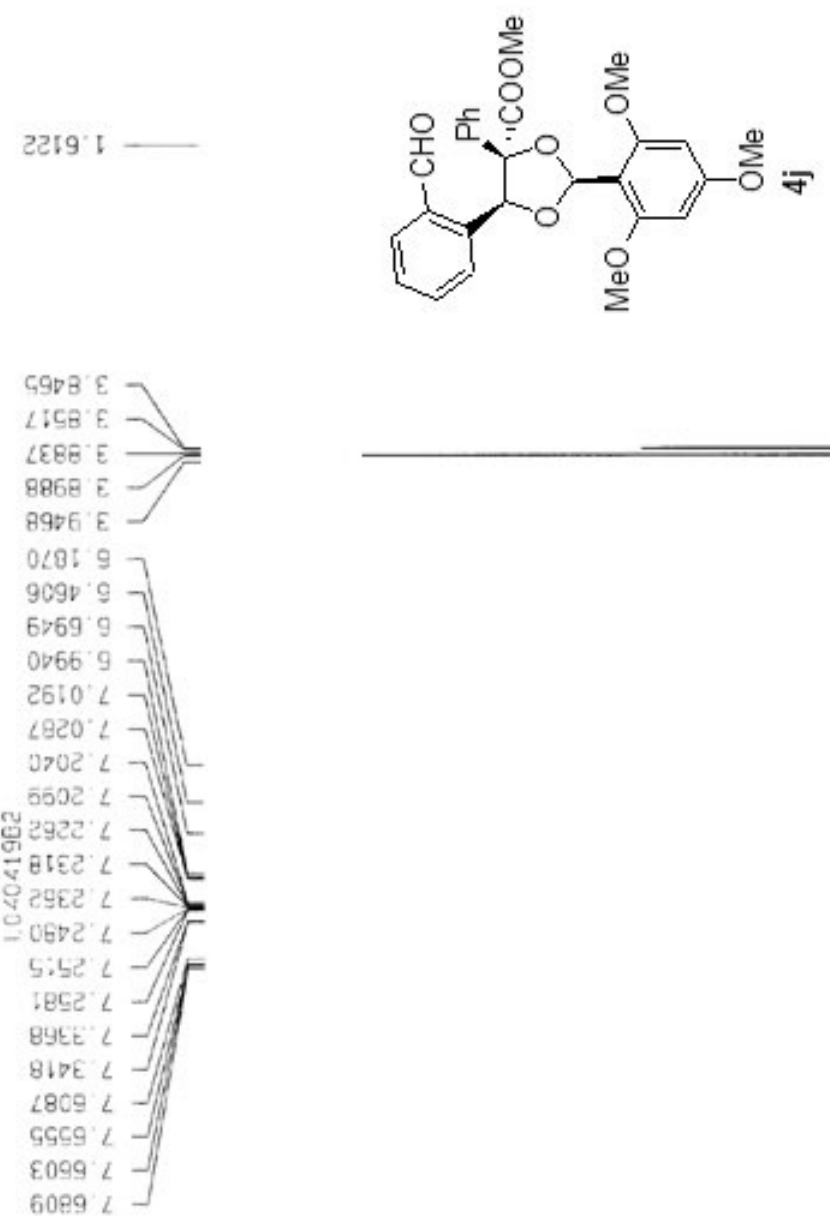

$8957^{\circ} 01-$

$20000^{\circ}$.

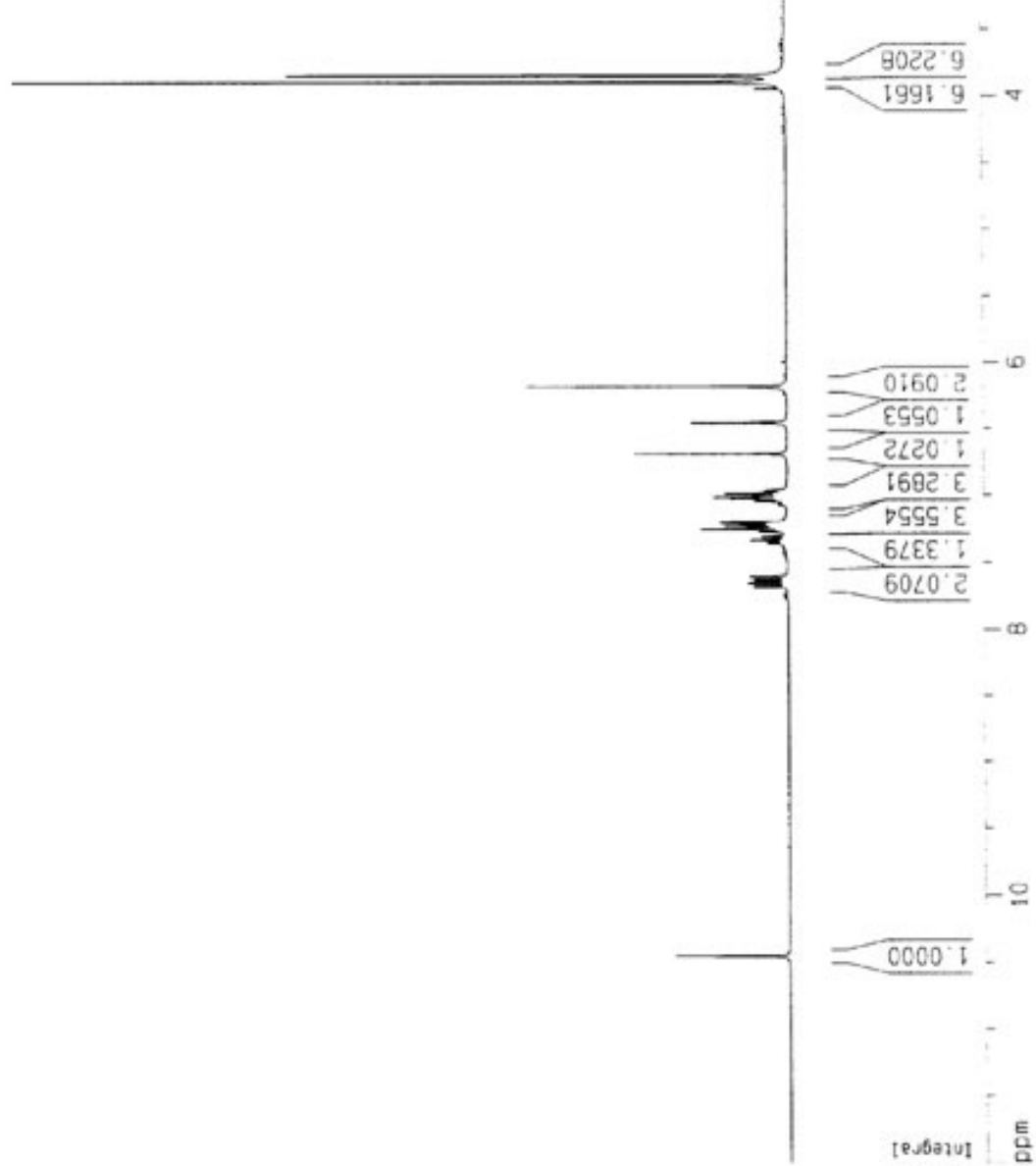


v2!: 0<smiles></smiles>

tè $\varepsilon s$

26055

$0 \angle 9 \div 9$

EDL $9 L$

$\angle S T \angle L$

065 L

๑૬ट $€ 8$

เबट $\angle 8$

998.06

$109^{\circ} 86$

I5I $201-$

079.951

$O L E$ LCI

SBL $\angle 2 L$

EจE $851-$

LSG $821-$

$\angle S E^{\prime} O E$ I-

เ86' $2 \varepsilon:$

896 गE:-

$\varepsilon 60$ 9ह:-

टIL BE:-

EणE 196 .

ShI ESt

OSD E.LT-

$8 \angle 8 \div 261-$

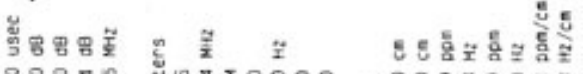

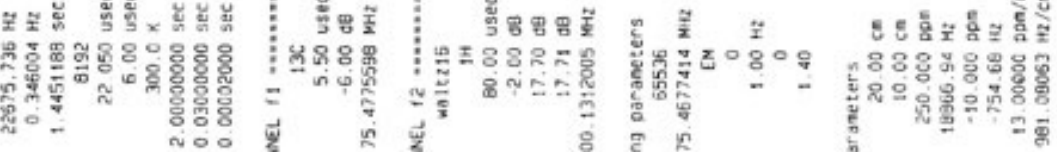

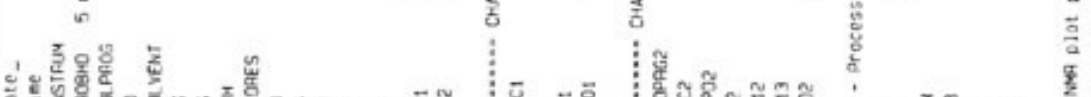

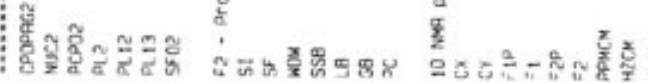

820:261 

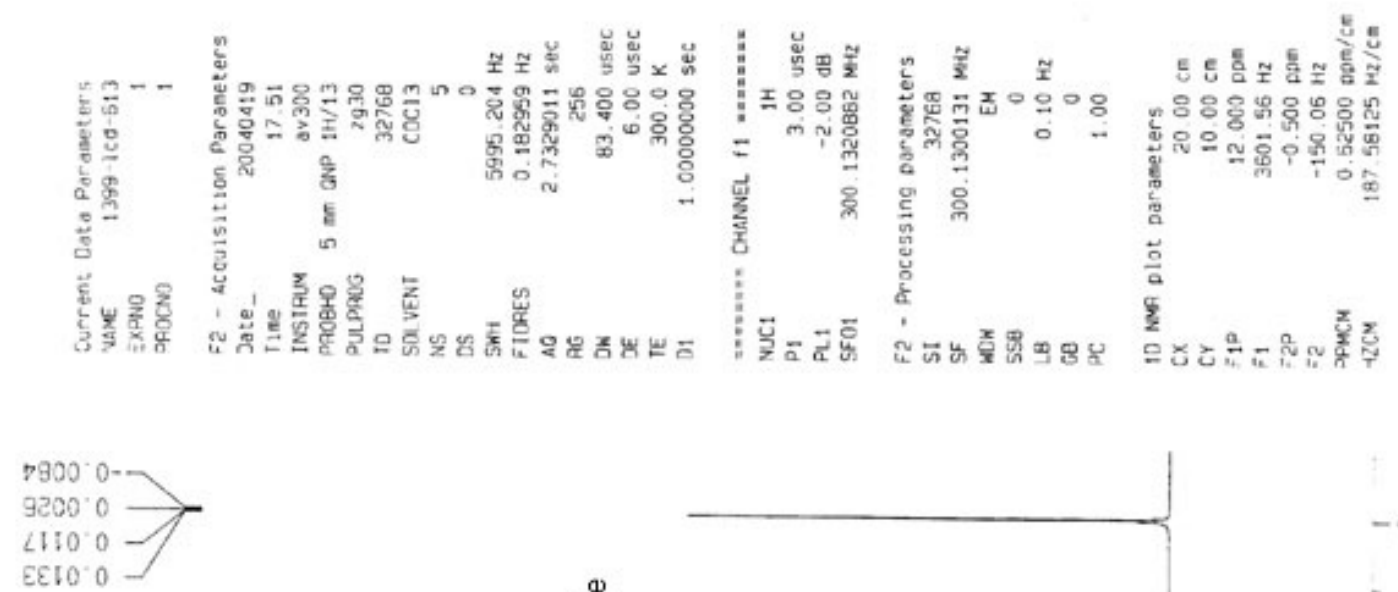

V855 E -

GeeL E

BECB E
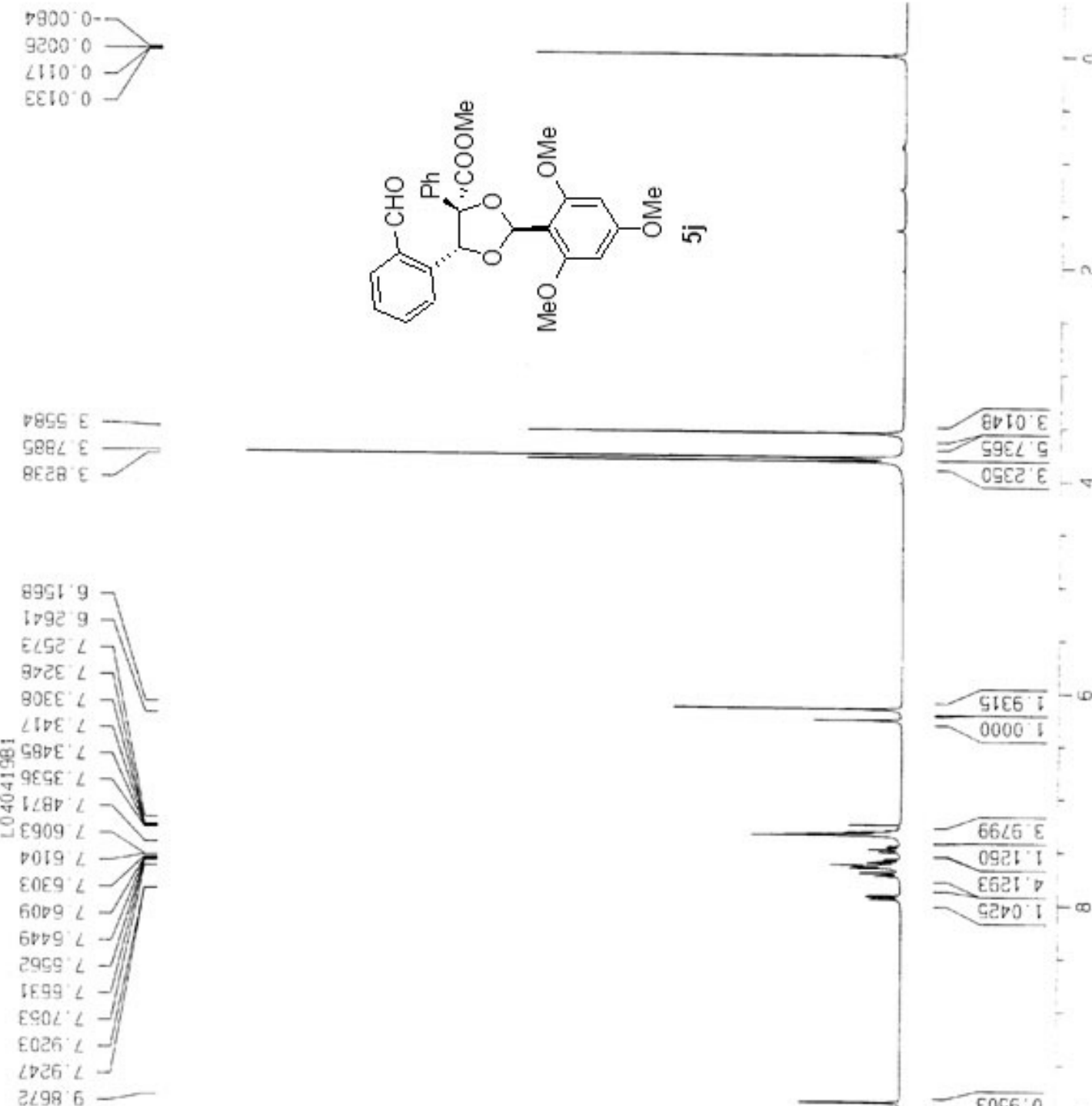


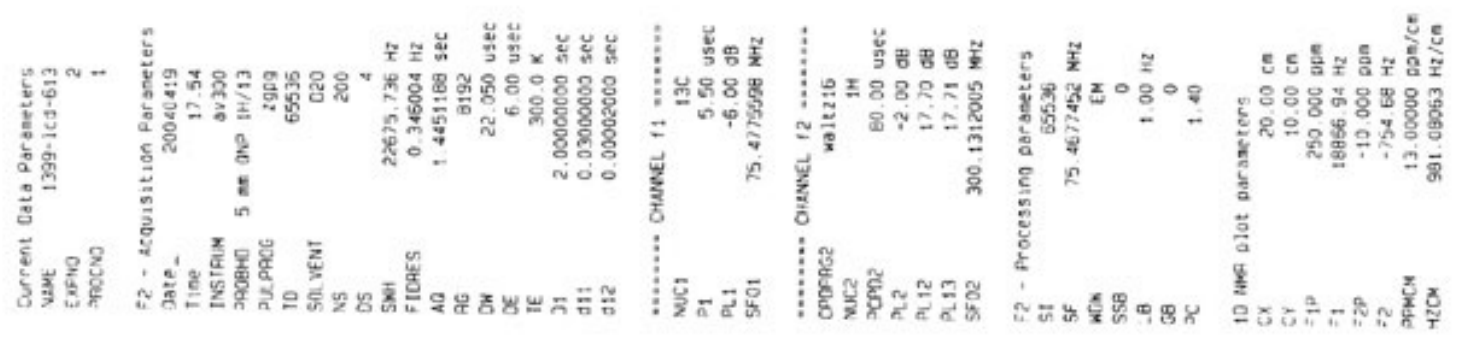

$580^{\circ} \mathrm{C}$<smiles>COc1cc(OC)c(C2OC(O)C(CO)O2)c(OC)c1</smiles>

9LE' $2 G-$
GOV'SS
$\angle D L G S$

टEL' $9 L$

9G) $\angle L$

$085 \angle L$

I $\angle 2$ EB

$959^{\circ} \mathrm{Eg}$

$\angle 6000$

600 00t-

$060.901-$

L19.92!

จ०ट. 851

$929^{\circ} 821-$

$8 \angle 0621-1$

$70 E$ EE -

टा9 $\nabla E I-$

L8S GEI-

99E ' $8 E$ :-

$569^{\circ} 09 \mathrm{Z}$

$209^{\circ} \mathrm{Cg}-$

ESO OLL -

$0 \angle G^{\circ} 06 \mathrm{~F}$

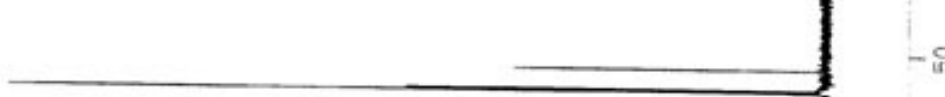

오 


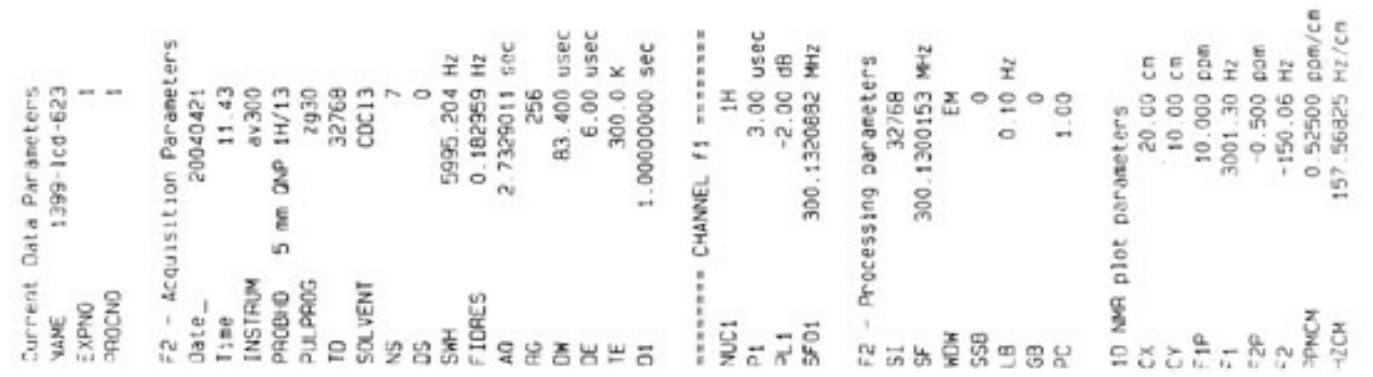

$2000^{\circ} 0-$

8500.0

LESG O

6209 :

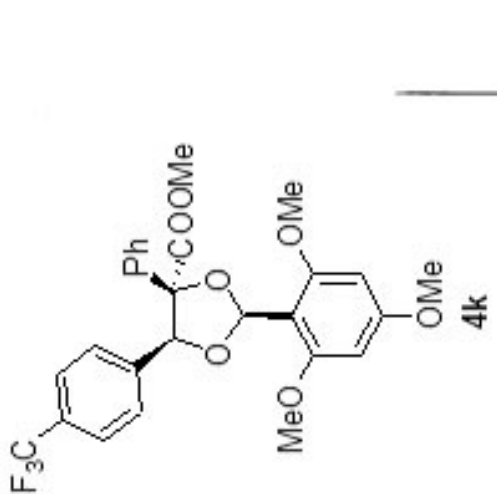

6อटล E

D००A E

DQPG E

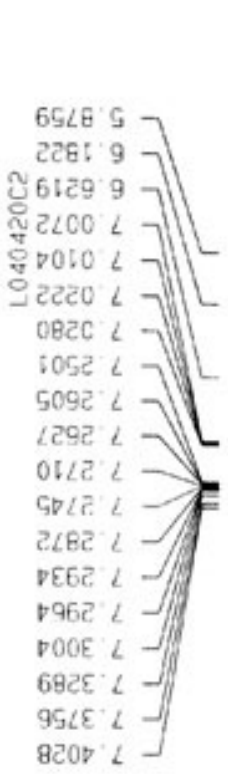

$\frac{\sqrt{69109}}{7751.9}-0$

wdC

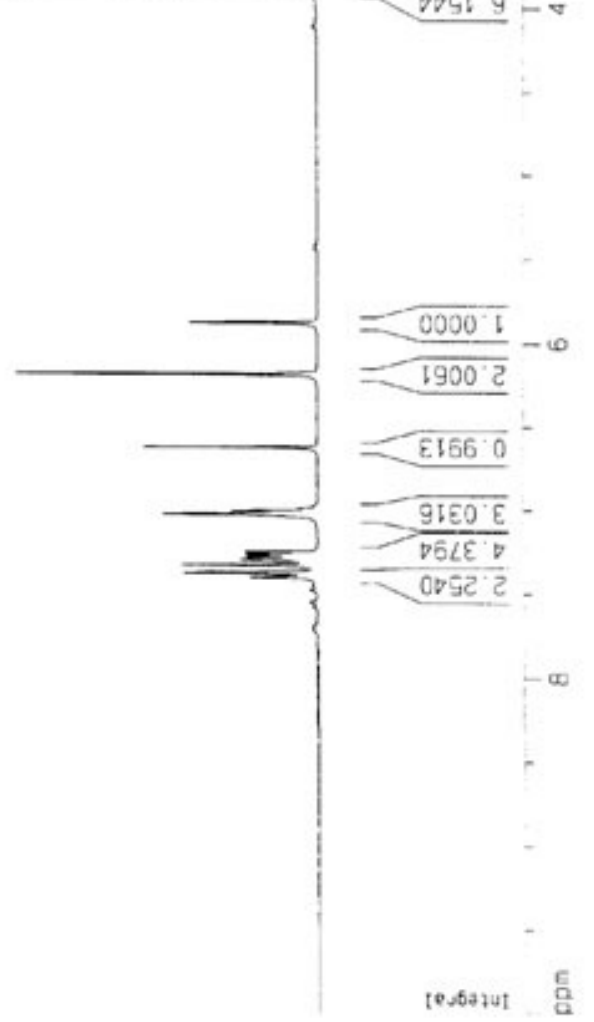




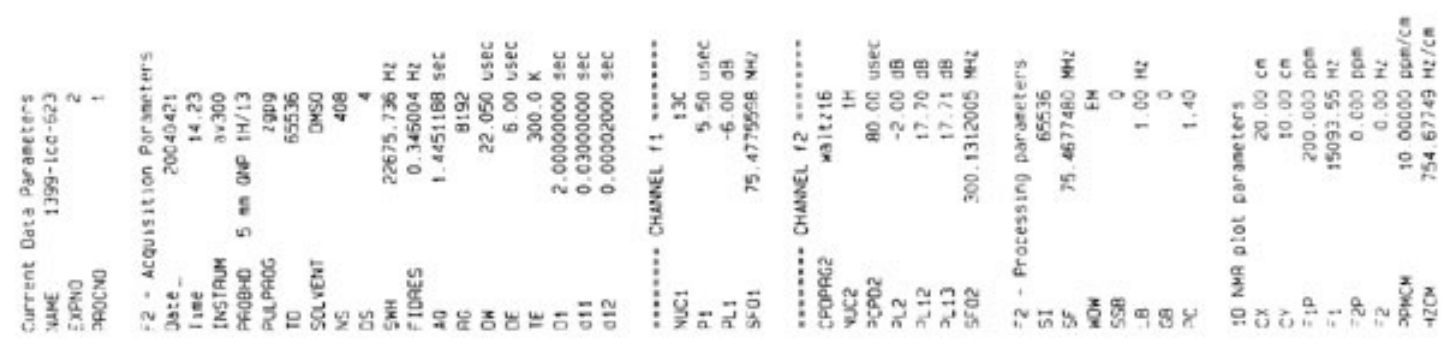

9000

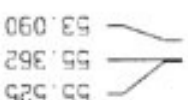

GटC $\mathrm{cS}$
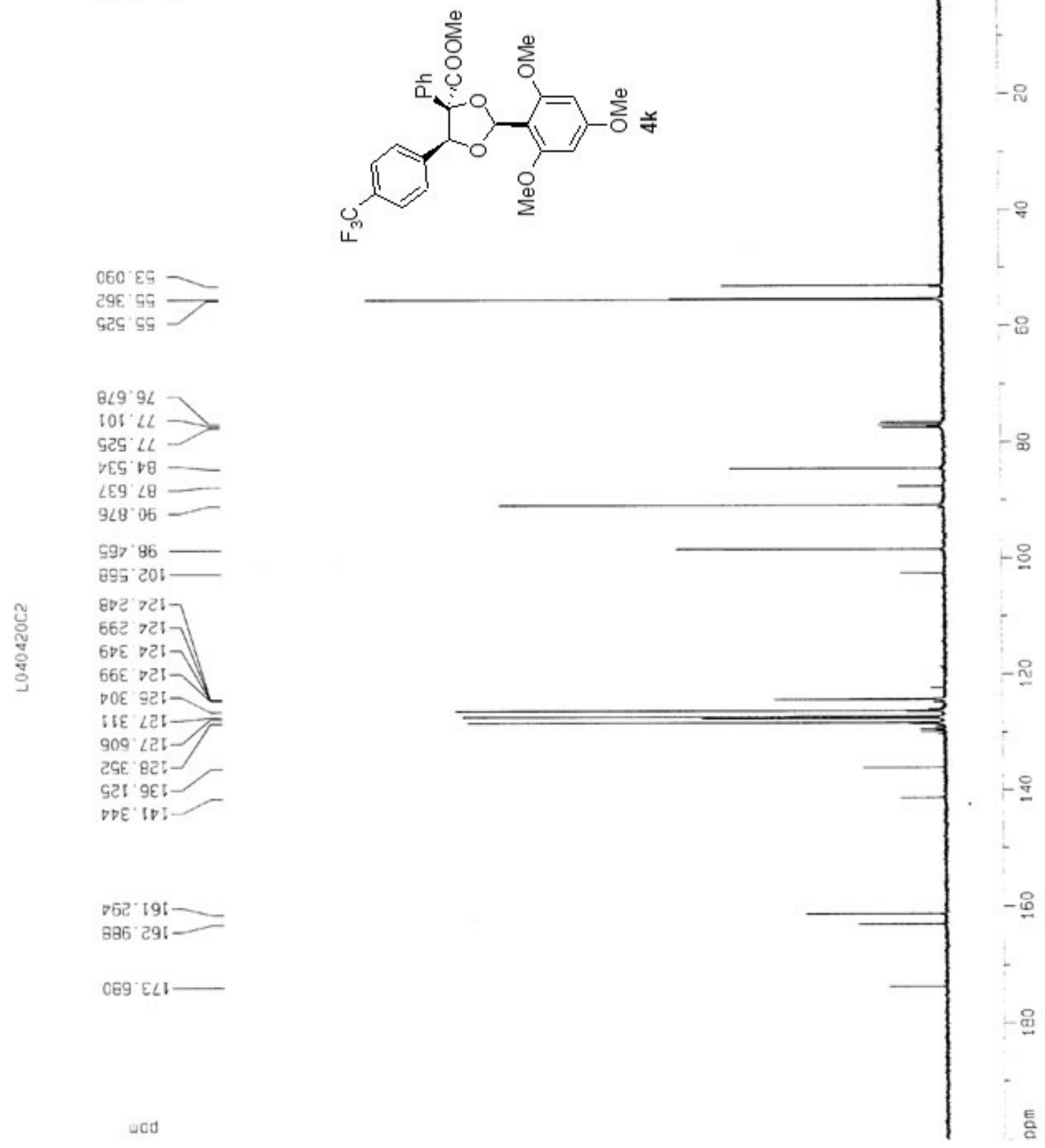


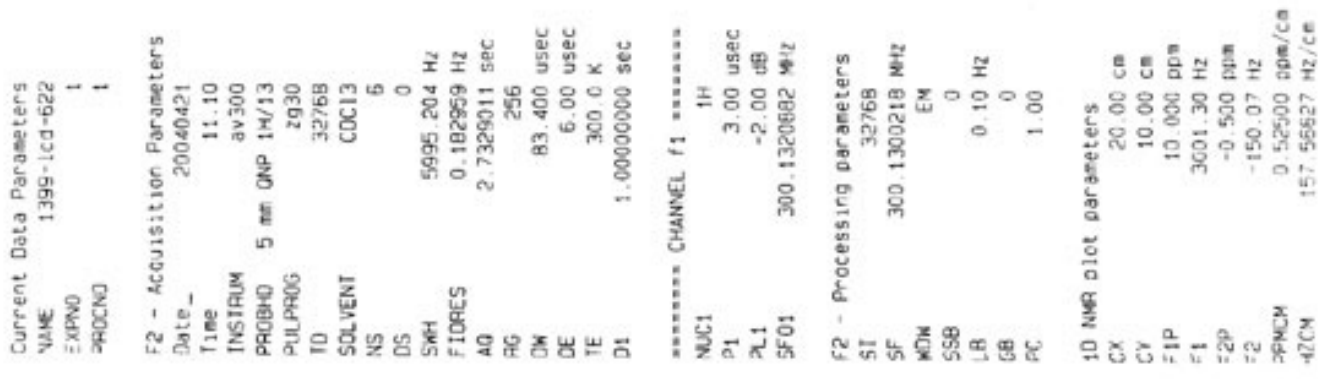

2000 0--<smiles>[O-]c1cc([O-])c(C2O[C@@H](c3ccc(O)cc3)[C@@]([O-])(O)O2)c(O)c1</smiles>

GGGD E

260L'E -

$8 \mathrm{EQ} L \mathrm{E}-$
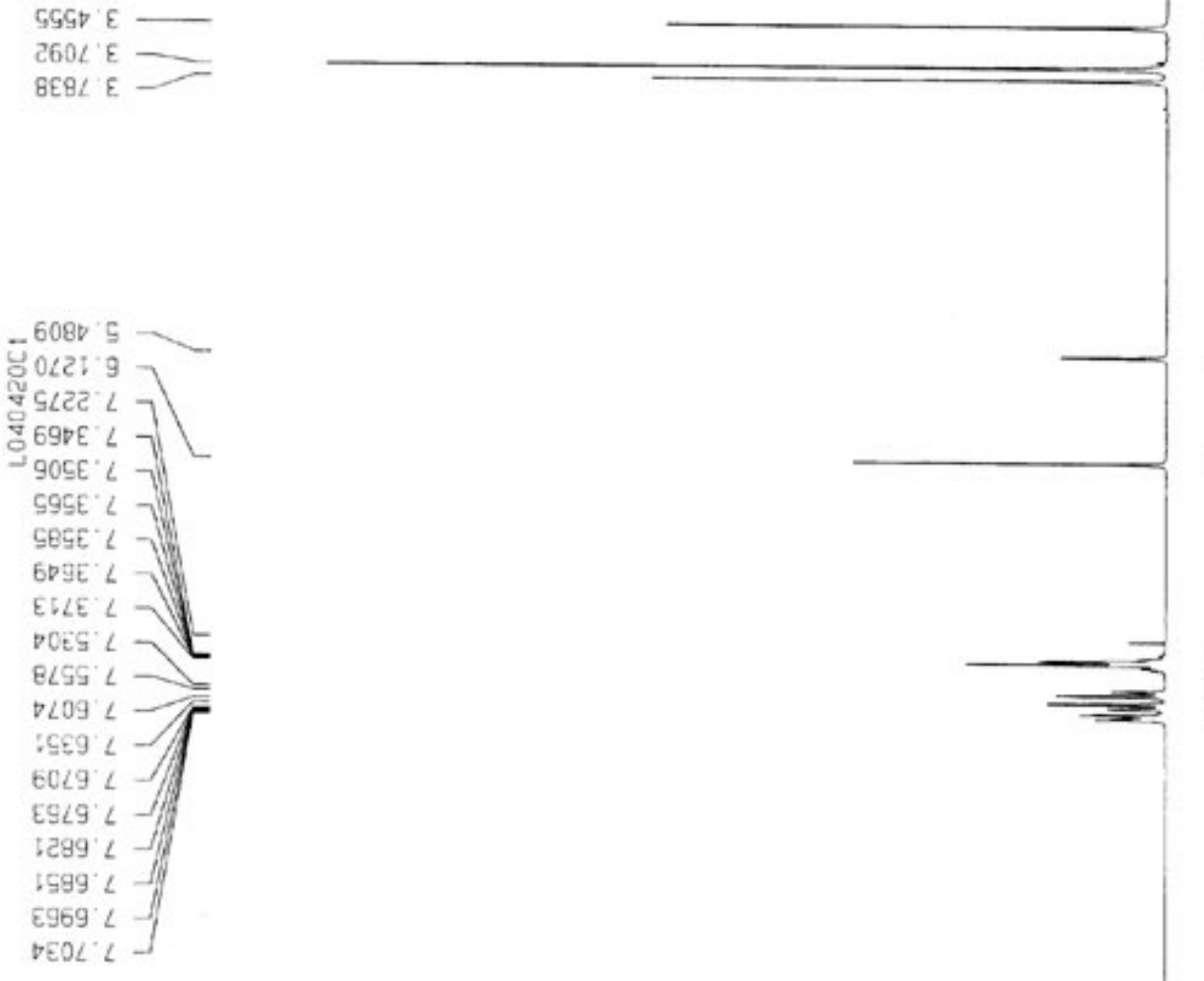

$=\frac{1000}{60}$

BEBL

vod

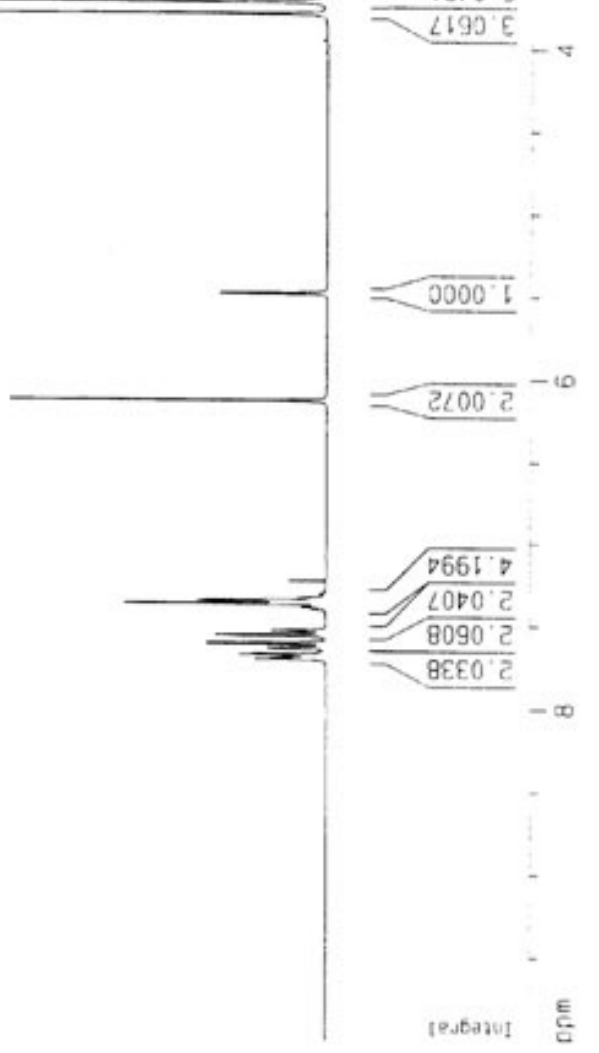




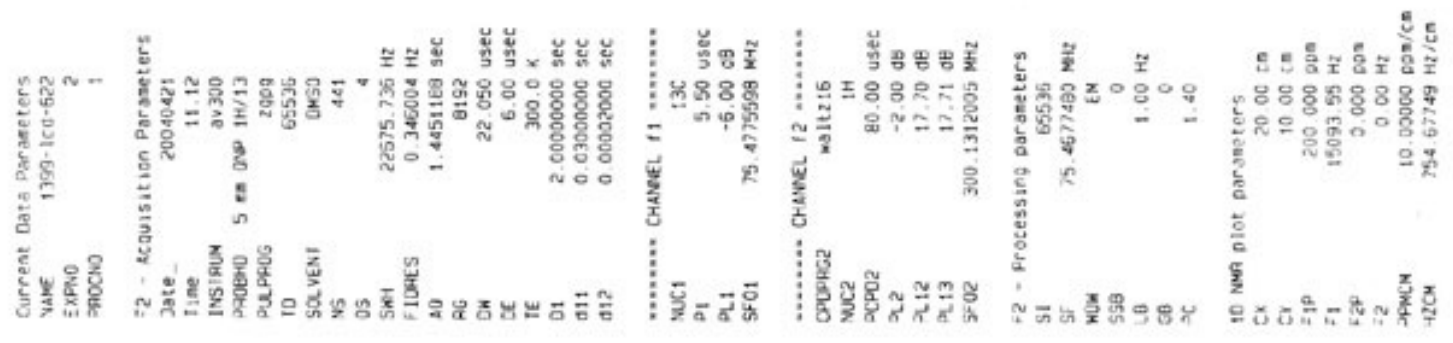

8200

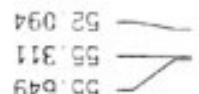

$6 \circ 9$ cद

$E D L ' 9 L$

घ9: $\angle L$

$26 \mathrm{C} \angle L$

09: $\angle B$

$9 \angle V$ 68

LEg 06

ESG 001

6Et $90 \mathrm{t}$.

दह० टटा

५S8 $\vee 21$

$\checkmark 06721$

म96 चटा

$200 \mathrm{gLl}$

500 get

069 921

मट० बटा-

6हट

OLट OEI-

8६9 Oह।

टाO $L E !-$

$8621771-$

2LE IOI

$102091-$

म: 291

EES OLI -

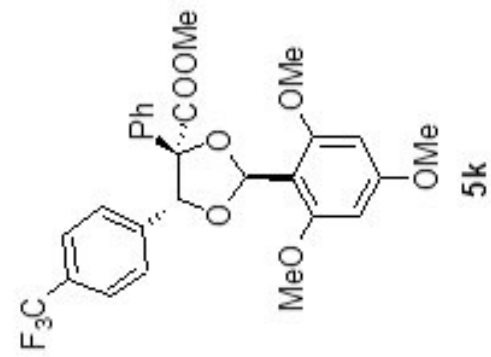

$-8$

i

$-\frac{9}{7}$

$-$

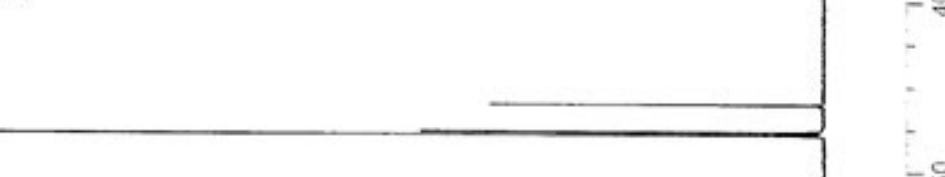

웅

프

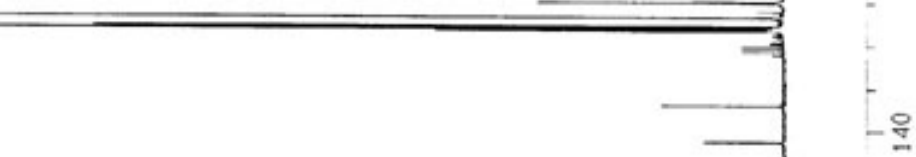

음

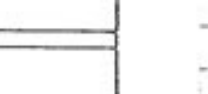

$\longrightarrow$

-욤 


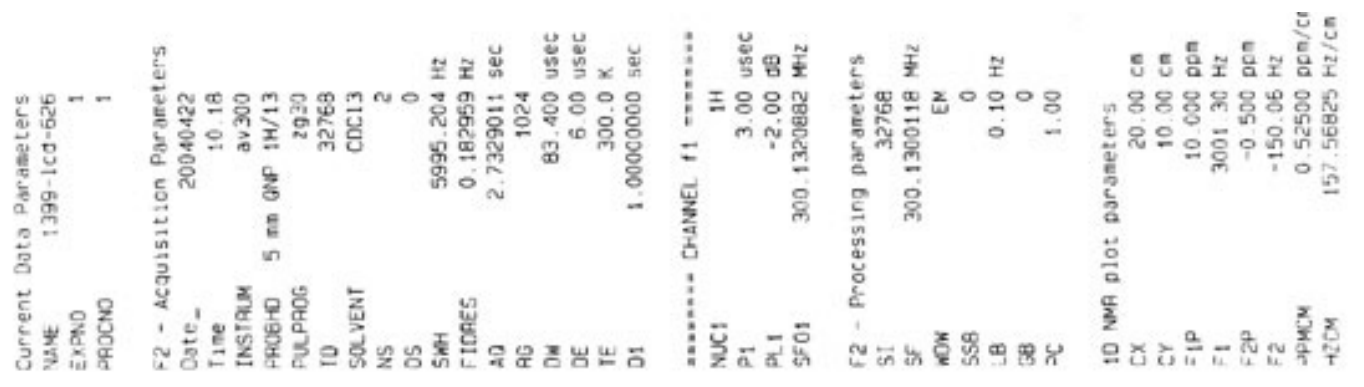

$2010^{\circ} 0-$

$8000^{\circ} 0$

9:1000
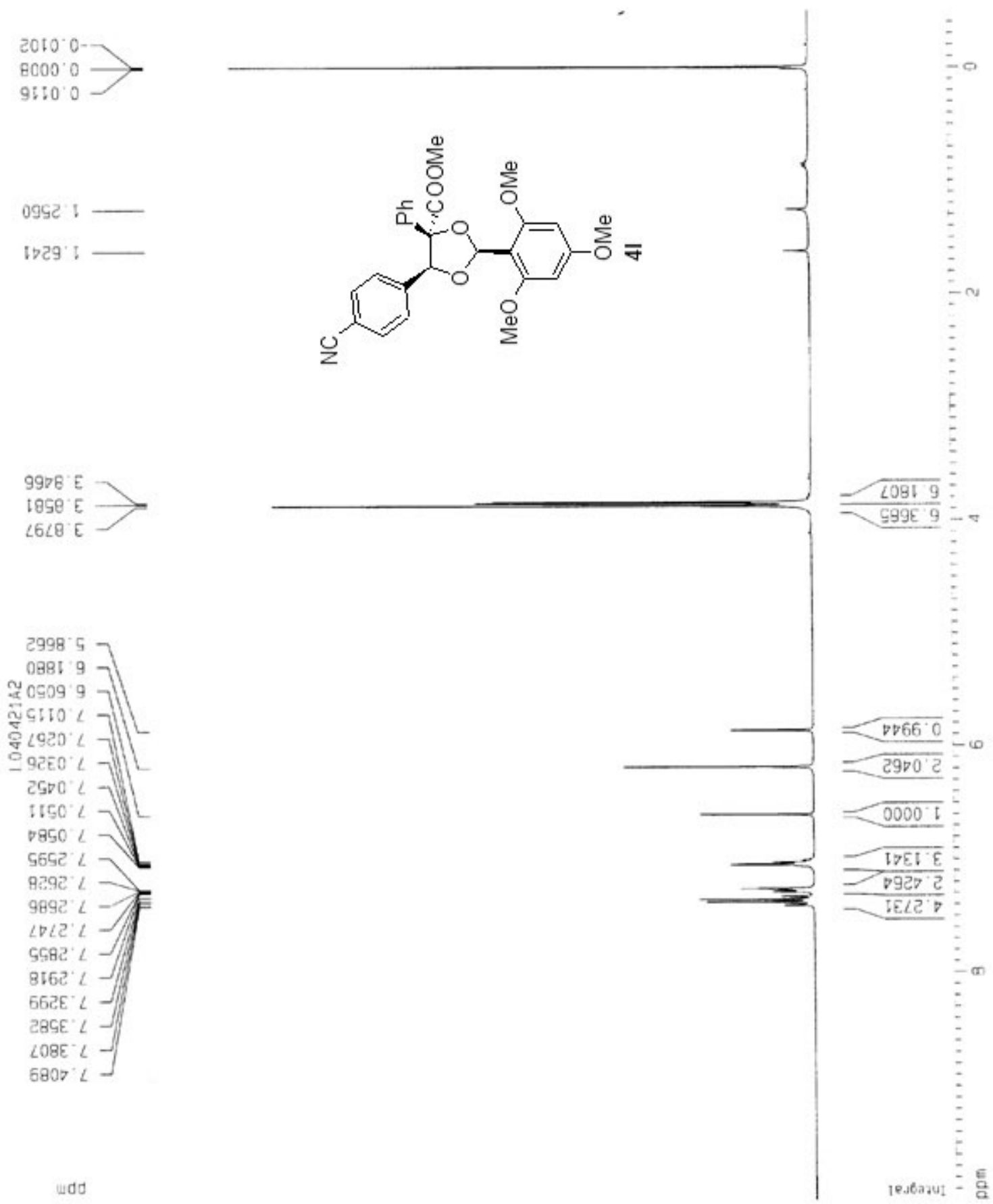

9978 E

1858

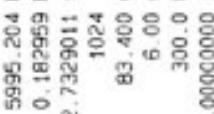

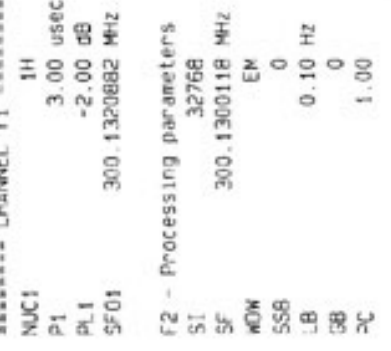

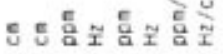

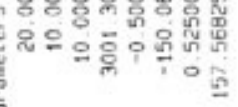

음

즌 


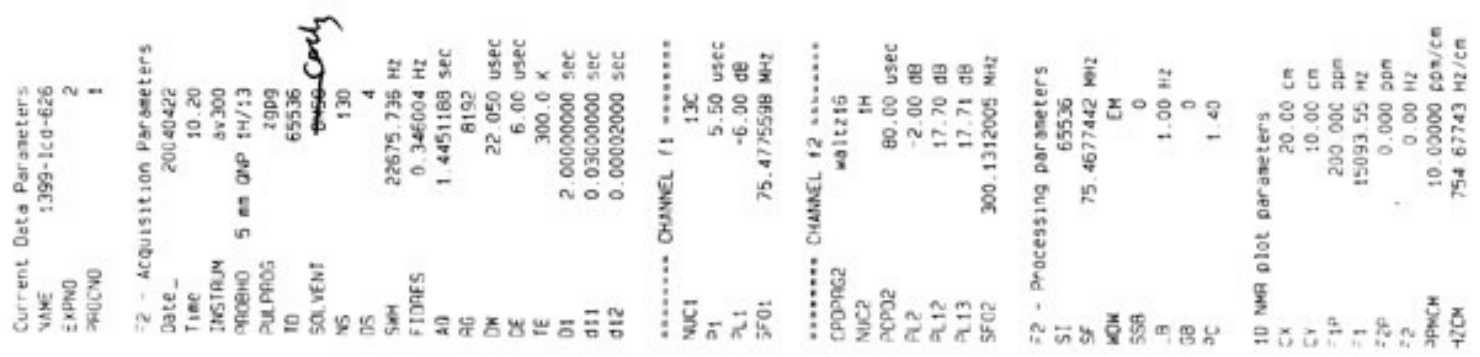

$060 \%$

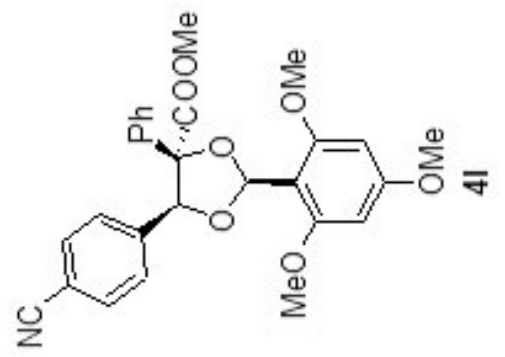

ट९ट ह9

:9V' GG

ह:9'

$82 L^{\circ} 9 L$

$59 \mathrm{I}^{\circ} \angle L-$

G28 $\angle A-$

$2 E 6^{\circ} 06$

$6 E 9 \cdot 86$

$612 t 5:-$

$\varepsilon 66^{\circ} \mathrm{Bt}$

५०ट. 92

$\angle E G^{\circ} \angle 2 !-$

$598^{\circ} \angle 5,-1$

$\angle \angle 9^{\circ} 8 \mathrm{BL}$.

OCE TEL

OG6 GE

$906 \mathrm{Cbt}$

$962 \cdot 19 t-$
$260 \cdot 891$

$\operatorname{tog}^{\circ} E L L-$

wdd 

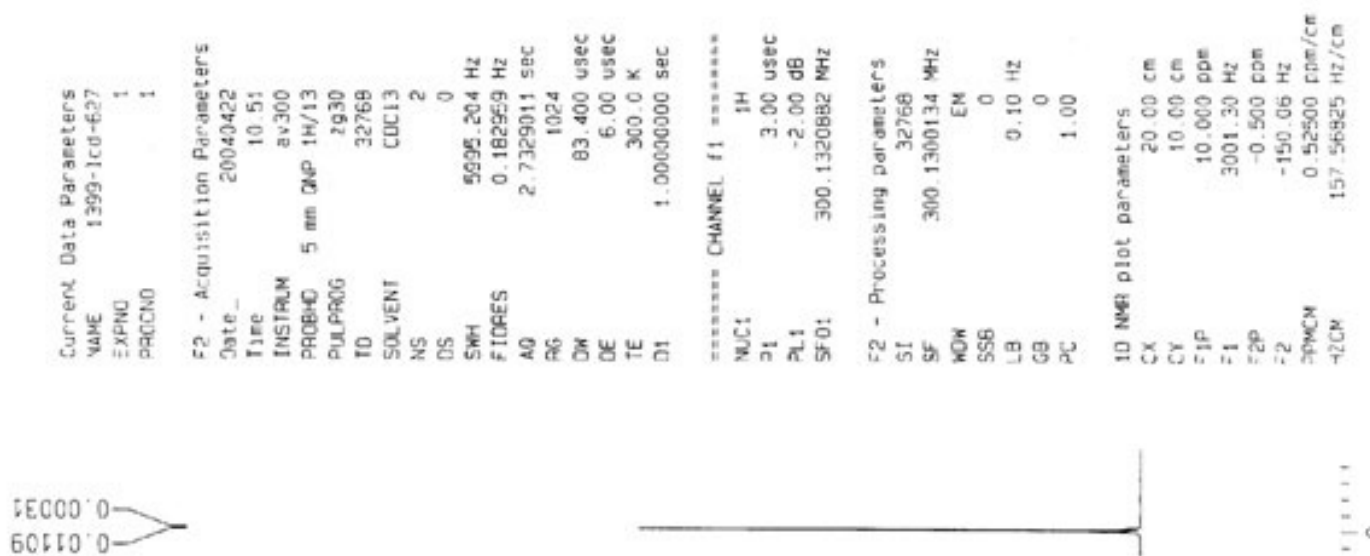

809291

¿2805:

$\angle 920 L \mathrm{E}$

SL8.6

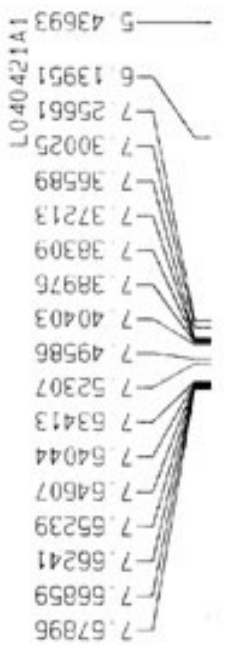

wod,

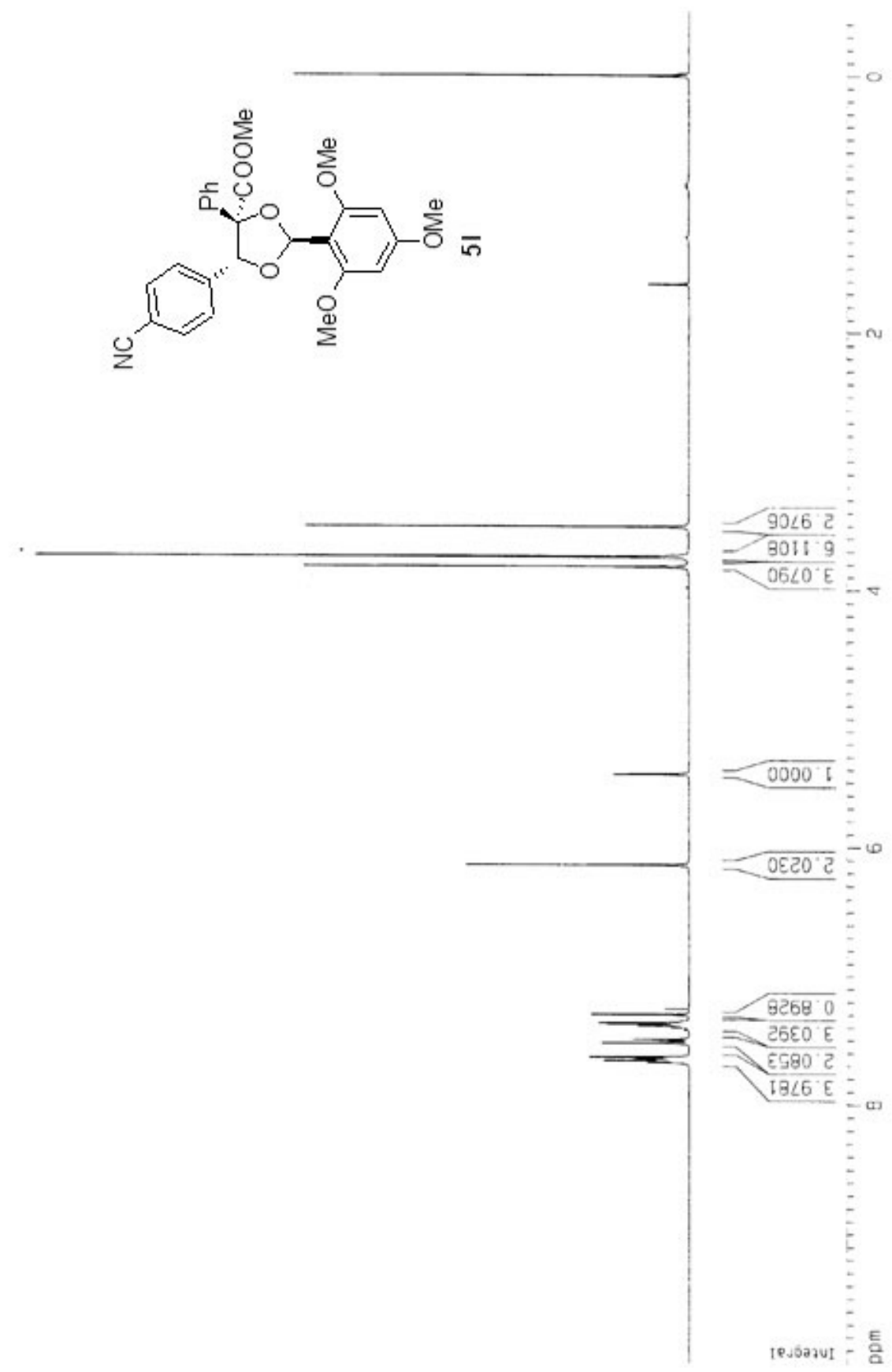




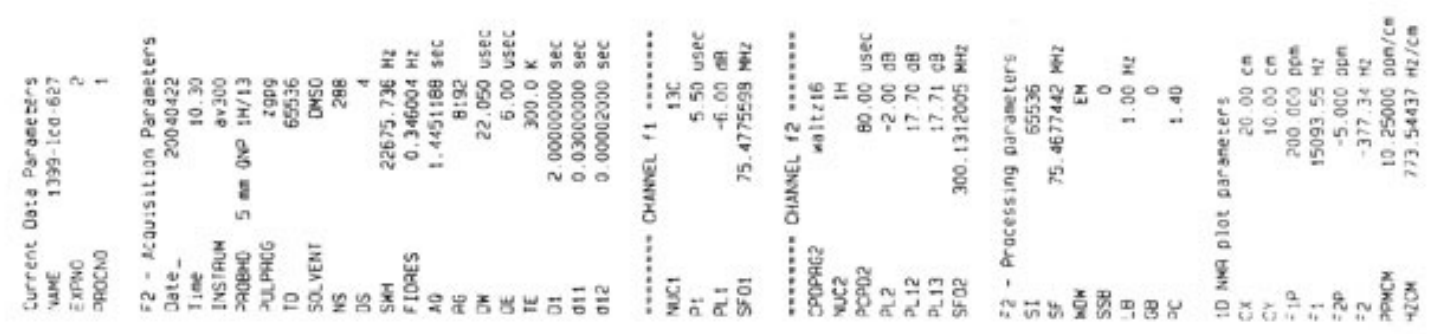

$860^{\circ} 0$<smiles>[CH]Oc1cc([O-])cc([O-])c1[C@@H]1O[C@@H](c2ccc([O-])cc2)C([O-])([O-])O1</smiles>

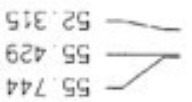

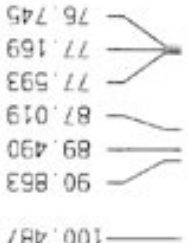

980.505

$890.211-$

$968.811-$

ट89 $921-$

$6 E 0.821-$

IIट $821-$

EIT BटI-

LIB TET

टBG 9E I-

E6S $201-$

$10 \angle 091-$

$102.291-$

$270^{\circ} 0 \angle 1-$

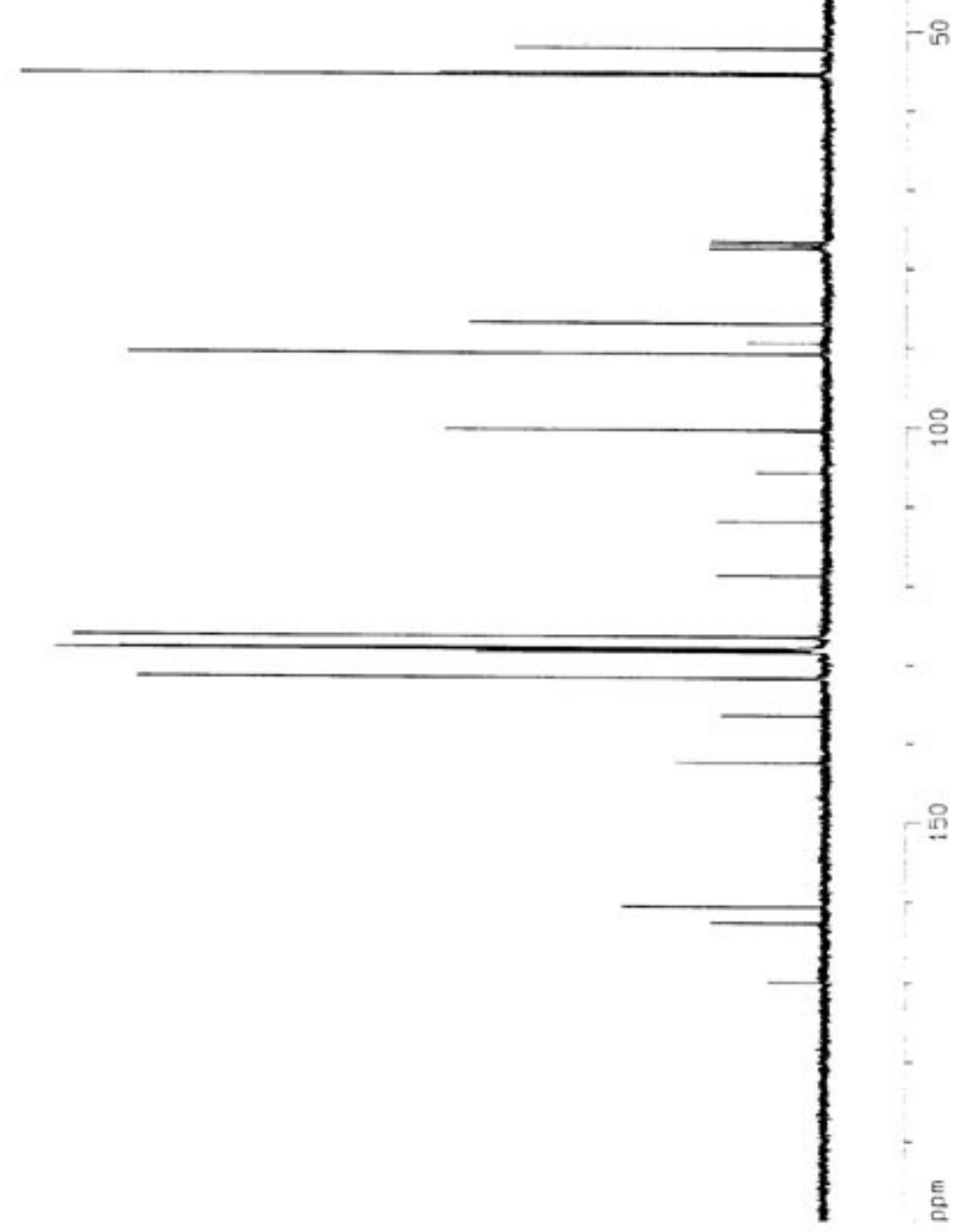




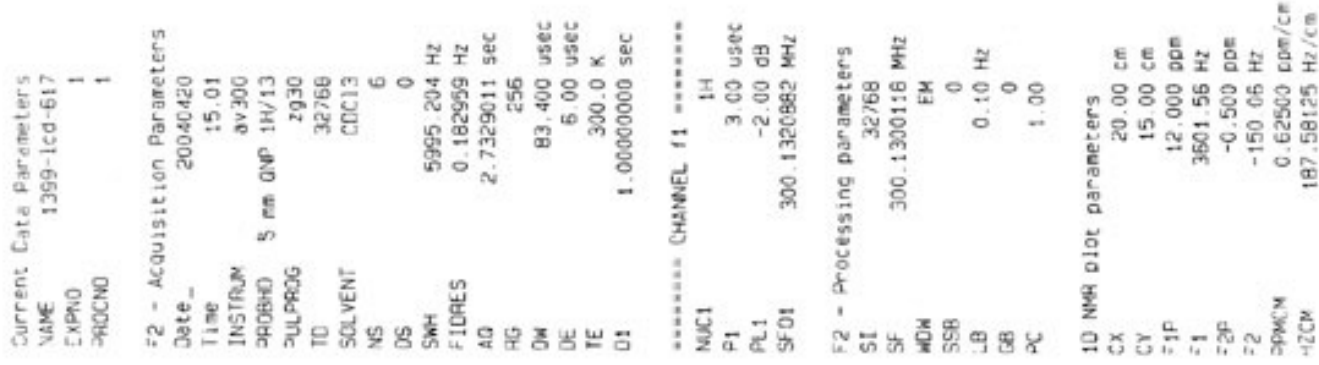

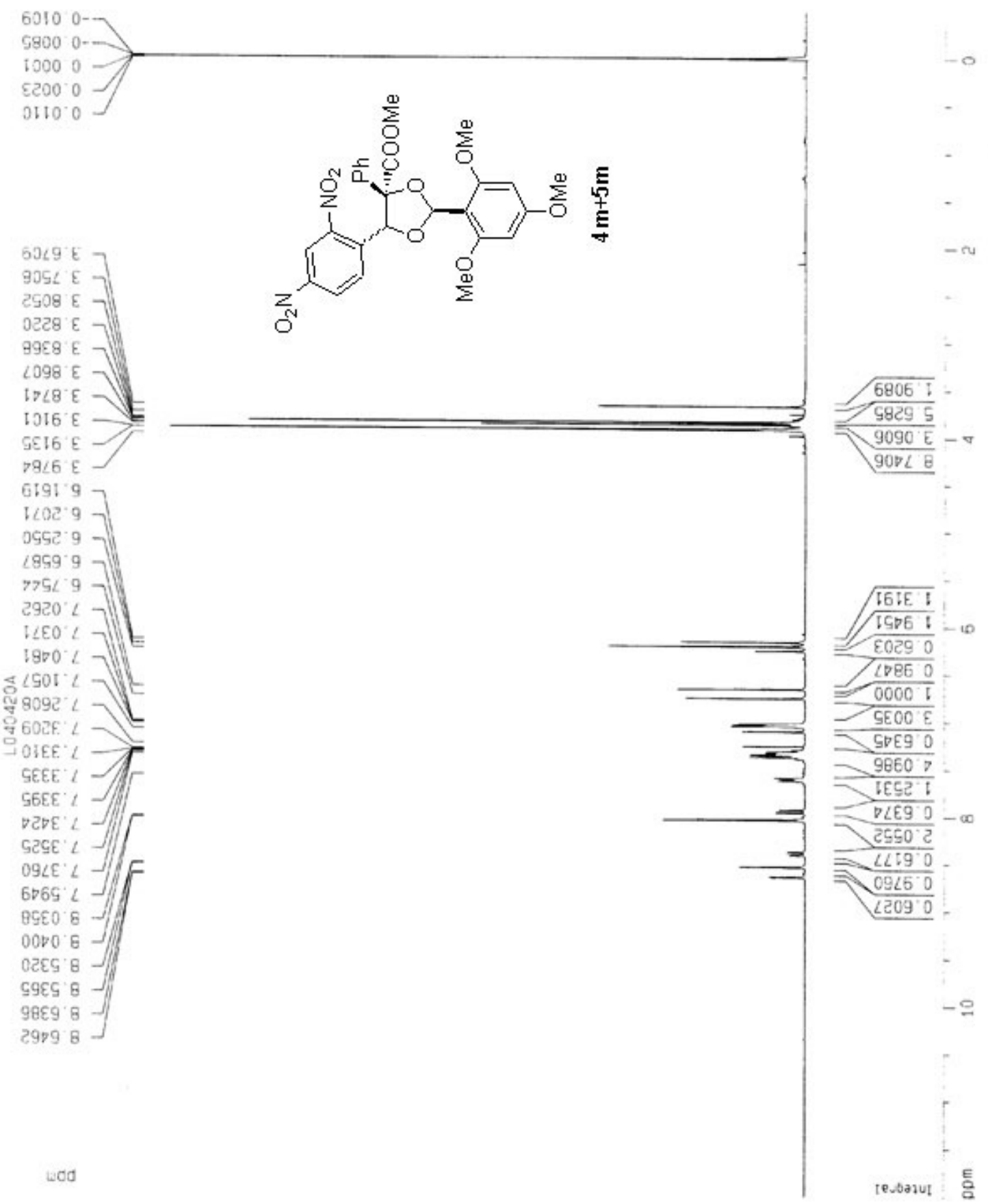



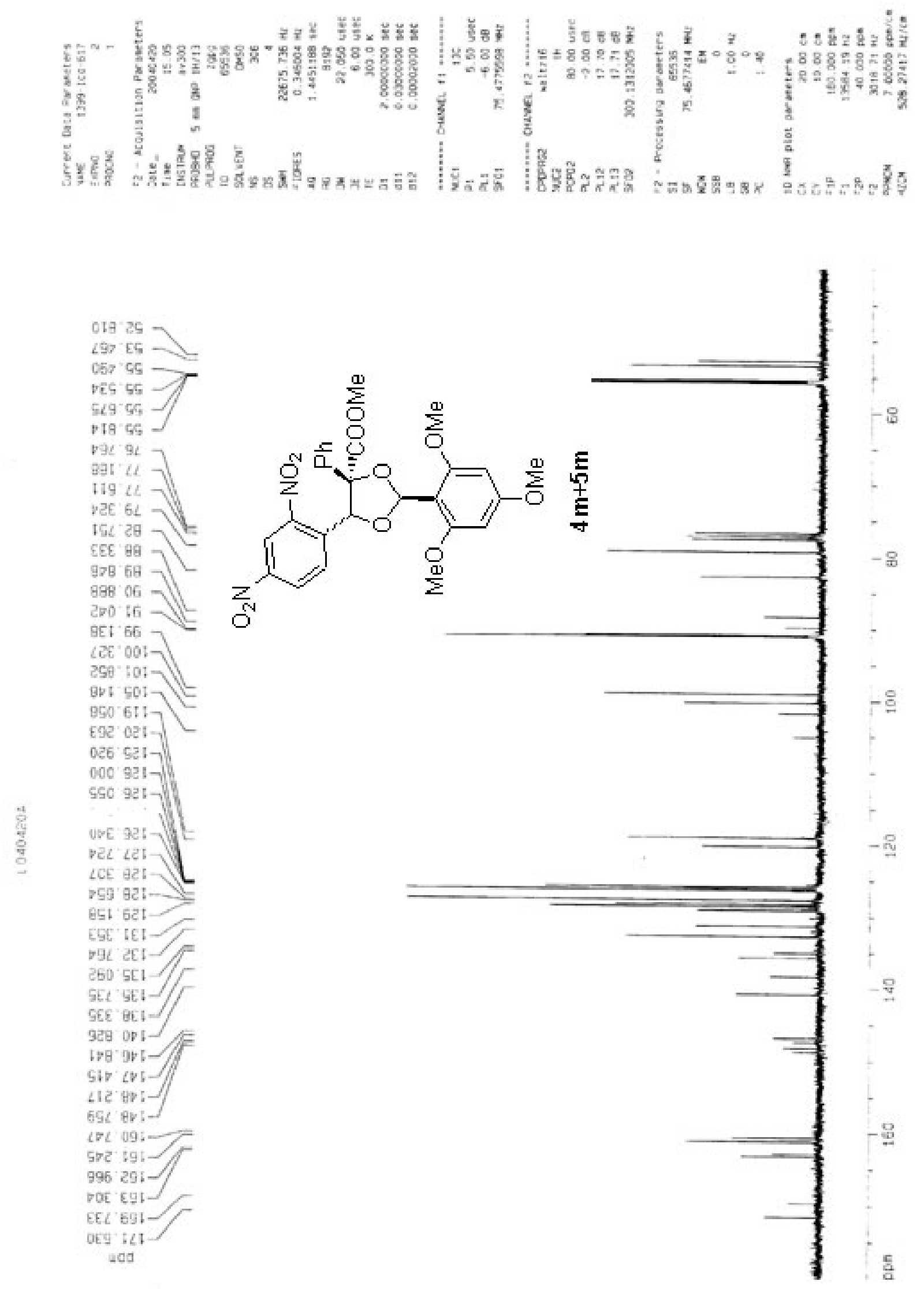


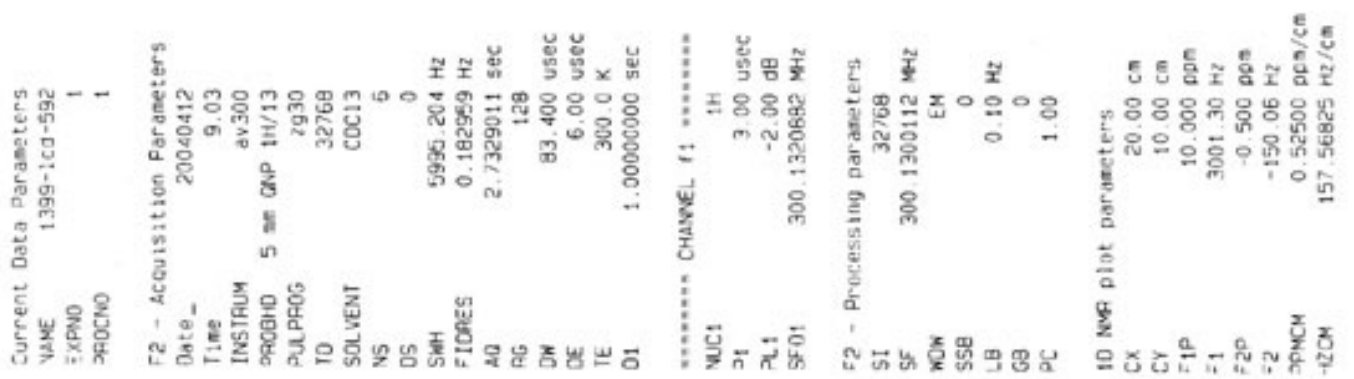

$820000->$

$55619:-$

06280

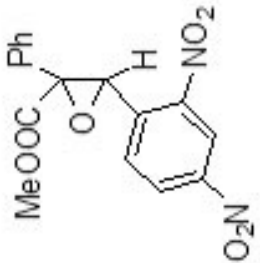

$\operatorname{coss} \angle>$

겅

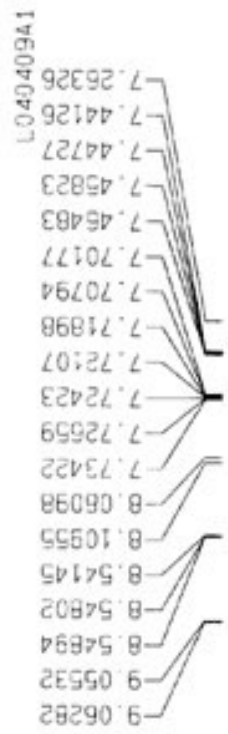

wdd

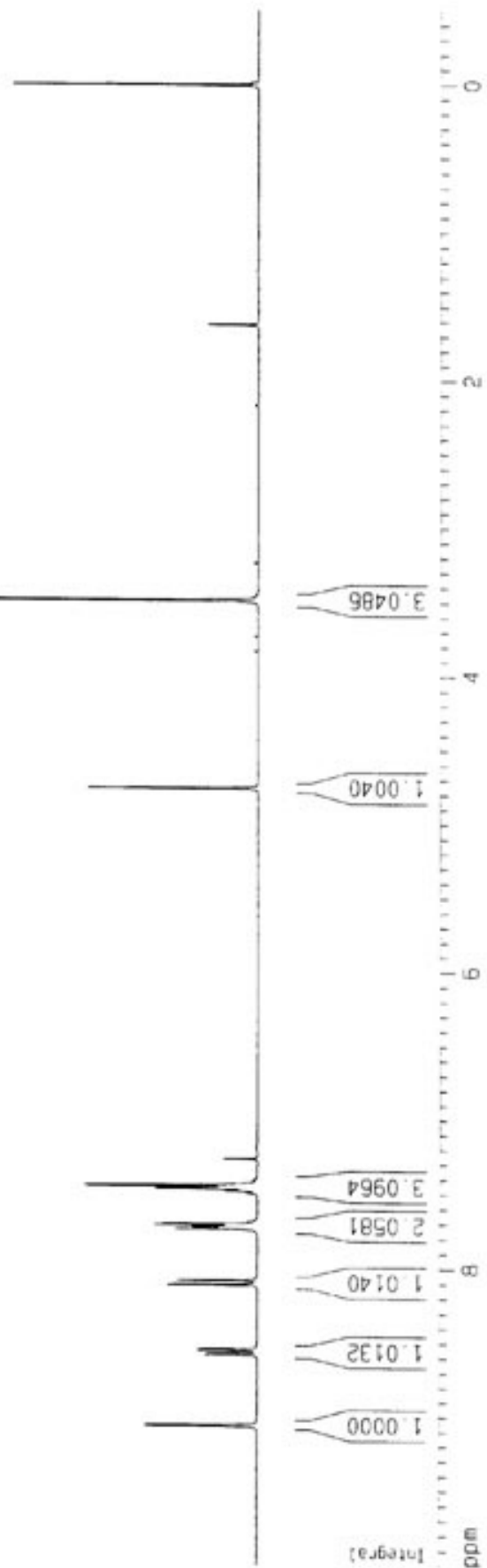


$801^{\prime} 0$
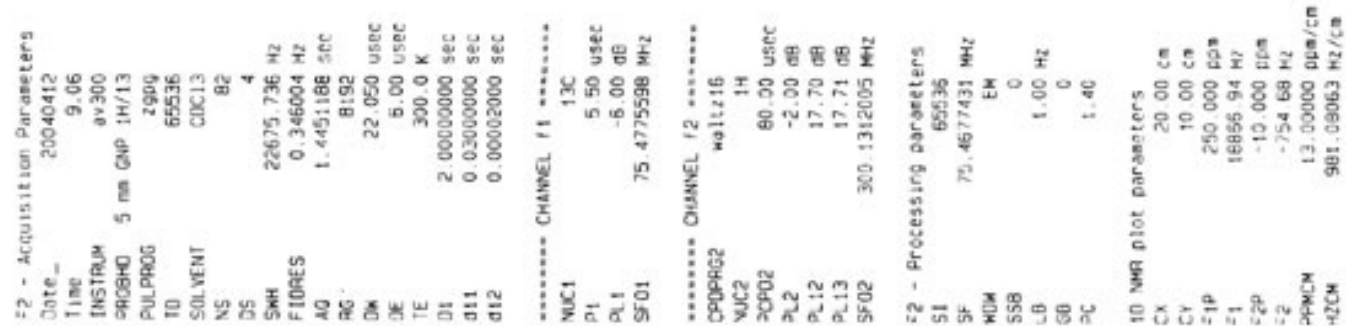

286 ट9

$120 \mathrm{Eg}$

$59 L^{\prime} 99$

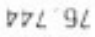

$B 91<L$

E.5 $\angle L$

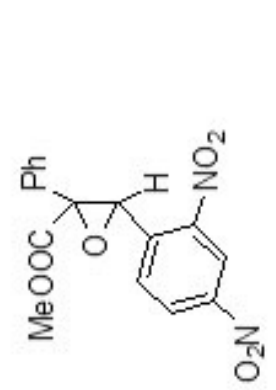

정

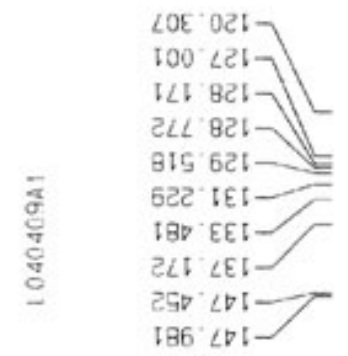

259. $991-$

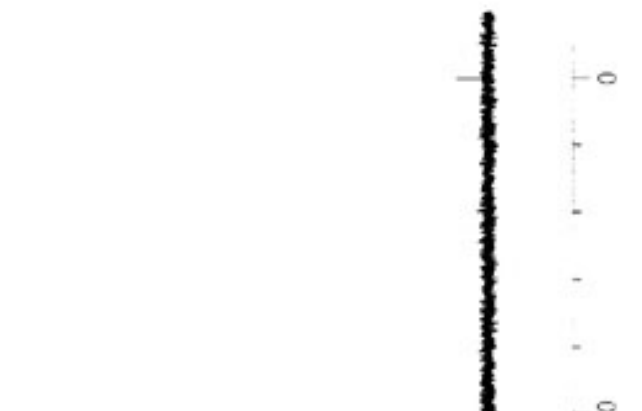

$-8$ 